\title{
Supersymmetric Deformations of Maximally Supersymmetric Gauge Theories.
}

\author{
M. V. Movshev \\ Stony Brook University \\ Stony Brook, NY 11794-3651, USA \\ A. Schwarz* \\ Department of Mathematics \\ University of California \\ Davis, CA 95616, USA
}

June 2, 2018

\section{Contents}

1 Introduction 3

2 Preliminaries $\quad 10$

2.1 Basic algebras . . . . . . . . . . . . . . 10

2.2 Calculation of the Lie algebra cohomology . . . . . . . . 17

2.3 The group $\operatorname{Spin}(10, \mathbb{C})$ and the space of pure spinors . . . . . 19

2.4 Euler characteristics . . . . . . . . . . . . . . . 21

3 Infinitesimal SUSY Deformations of $\mathcal{L}_{S Y M}$

${ }^{*}$ The work of both authors was partially supported by NSF grant No. DMS 0505735 and by grants DE-FG02-90ER40542 and PHY99-0794 
4 The Homological Approach to Infinitesimal Deformations

5 Calculation of the Cohomology 37

6 The BV formalism: a geometric approach 48

$\begin{array}{lll}7 & \text { Formal SUSY deformations } & 62\end{array}$

A $\mathbf{L}_{\infty}$ and $\mathrm{A}_{\infty}$ algebras

B Duality $\quad 80$

$\mathrm{C}$ On the relation of the Lie algebra and the $\mathrm{BV}$ approaches to the deformation problem

D The $\mathrm{L}_{\infty}$ action of the supersymmetry algebra in the BV formulation

E Calculation of the hypercohomology 92

F Construction of the supersymmetric deformations 94

G A deformation complex $\quad 97$

H Dimensional reductions of SYM 101

I Representation rings $\quad 105$

\begin{abstract}
We study supersymmetric and super Poincaré invariant deformations of ten-dimensional super Yang-Mills theory and of its dimensional reductions. We describe all infinitesimal super Poincaré invariant deformations of equations of motion of ten-dimensional super Yang-Mills theory and its reduction to a point; we discuss the extension of them to formal deformations. Our methods are based on homological algebra, in particular, on the theory of L-infinity and A-infinity algebras. The exposition of this
\end{abstract}


theory as well as of some basic facts about Lie algebra homology and Hochschild homology is given in appendices.

\section{Introduction}

The superspace technique is a very powerful tool of construction of supersymmetric theories. However this technique does not work for theories with large number of supersymmetries. It is possible to apply methods of homological algebra and formal non-commutative geometry to prove existence of supersymmetric deformations of gauge theories and give explicit construction of them. In this paper we discuss results obtained by such methods in the analysis of supersymmetric (SUSY) deformations of 10-dimensional SUSY YM-theory (SYM theory) and its dimensional reductions.

These deformations are quite important from the viewpoint of string theory. The D-brane action in the first approximation is given by dimensional reduction of ten-dimensional SYM theory; taking into account the $\alpha^{\prime}$ corrections we obtain SUSY deformation of this theory. (More precisely, we obtain a power series with respect to $\alpha^{\prime}$ specifying a formal deformation of the theory at hand.)

Our approach is closely related to pure spinors techniques; it seems that it could be quite useful to understand better the pure spinor formalism in string theory constructed by Berkovits [5].

Recall that a $\mathrm{U}(N)$ gauge potential $A_{i}(x)$ and a chiral spinor $\chi^{\alpha}$ are the fields of the SYM-theory. In component form the action functional of SYM-theory looks as follows:

$$
S_{S Y M}(A, \chi)=\int \mathcal{L}_{S Y M} d^{10} x=\int \operatorname{tr}\left(\frac{1}{4} F_{i j} F_{i j}+\frac{1}{2} \Gamma_{\alpha \beta}^{i} \chi^{\alpha} \nabla_{i} \chi^{\beta}\right) d^{10} x
$$

$\nabla_{i}=\frac{\partial}{\partial x^{i}}+A_{i}(x)$ are covariant derivatives, $\chi^{\alpha}$ are chiral spinors with values in the adjoint representation, $F_{i j}=\left[\nabla_{i}, \nabla_{j}\right]$ is the curvature 1

\footnotetext{
${ }^{1}$ In this text by default small Roman indices $i, j$ run over $1, \ldots, 10$, Greek indices $\alpha, \beta, \gamma$ run over $1, \ldots, 16$

In the above formula $A_{1}, \cdots A_{10}$ and $\chi^{1}, \cdots, \chi^{16}$ denote fields on ten-dimensional space
} 
Let $Y$ be a linear combination of products of covariant derivatives of the curvature $F_{i j}$ and spinor fields $\chi^{\alpha}$. By construction $Y$ is is a section of the adjoint bundle. The function $A_{i}, \chi^{\alpha} \rightarrow Y\left(A_{i}, \chi^{\alpha}\right)$ commutes with the gauge group action.

We shall refer to such $Y$ as a gauge-covariant local expression. A local gaugeinvariant expression is by definition $\operatorname{tr}(Y)$ with local gauge-covariant $Y$. In this paper we shall consider deformation that can be described by action functionals of the form

$$
\int \mathcal{L}_{S Y M}+\sum_{i \geq 1} \int \operatorname{tr}\left(Y_{i}\right) \epsilon^{i}
$$

where $\operatorname{tr} Y_{i}$ are gauge invariant, $\epsilon$ is a formal deformation parameter.

In our approach the integrals are invariant with respect to some field transformation iff the variation of the integrand is a total derivative. We consider only deformations that can be applied simultaneously to gauge theories with all gauge groups $\mathrm{U}(N)$ where $N$ is an arbitrary positive integer. This means that we miss some of the deformations (e.g. certain deformations of the abelian gauge theory) that are defined only for a finite range of $N$.

Our methods can be applied to supersymmetric deformations of dimensional reductions of SYM theory. A SYM deformation after reduction defines a deformation of the corresponding reduced theory. However not all deformations of the reduced theory are of this kind. We shall give a complete description of SUSY- deformations of the full SYM theory and of its reduction to $\mathrm{D}=0$.

Let us list explicit formulas for the action of supersymmetry generators and translations on fields of SYM-theory. The Lie algebra of supersymmetry acts

$\mathbb{R}^{10}$ taking values in complex $N \times N$-matrices. (We do not impose the hermiticity condition; in our approach this condition appears only in the choice of real slice in the space of fields that we should use in the definition of the functional integral of the quantum theory.)

. We shall freely raise and lower Roman indices with ten-dimensional metric $\left(d x^{i}\right)^{2}$. We use Einstein summation convention over repeated indices. 
on gauge equivalence classes of solutions of equations

$$
\begin{aligned}
& \sum_{i=1}^{10} \nabla_{i} F_{i m}-\frac{1}{2} \sum_{\alpha \beta=1}^{16} \Gamma_{\alpha \beta}^{m}\left[\chi^{\alpha}, \chi^{\beta}\right]=0 \quad m=1, \ldots, 10 \\
& \sum_{\beta=1}^{16} \sum_{i=1}^{10} \Gamma_{\alpha \beta}^{i} \nabla_{i} \chi^{\beta}=0 \quad \alpha=1 \ldots 16
\end{aligned}
$$

The supersymmetry operators $\theta_{\alpha}$ are defined by formulas

$$
\begin{aligned}
& \theta_{\alpha} A_{i}=\Gamma_{\alpha \beta i} \chi^{\beta} \\
& \theta_{\alpha} \chi^{\beta}=\Gamma_{\alpha}^{\beta i j} F_{i j}
\end{aligned}
$$

Denote by $D_{i}$ the lift of the space-time translation $\partial / \partial x^{i}$ to the space of fields $\left(A_{i}, \chi^{\alpha}\right)$. The lift is defined only up to gauge transformation. We fix the gauge freedom in a choice of $D_{i}$ requiring that

$$
\begin{aligned}
& D_{i} A_{j}=F_{i j} \\
& D_{i} \chi^{\alpha}=\nabla_{i} \chi^{\alpha}
\end{aligned}
$$

Infinitesimal symmetries $\theta_{\alpha}$ satisfy

$$
\begin{aligned}
& {\left[\theta_{\alpha}, \theta_{\beta}\right]=\Gamma_{\alpha \beta}^{i} D_{i}} \\
& {\left[\theta_{\alpha}, D_{i}\right] A_{k}=-\Gamma_{\alpha \beta i} \nabla_{k} \chi^{\beta}} \\
& {\left[\theta_{\alpha}, D_{i}\right] \chi^{\gamma}=\Gamma_{\alpha \beta i}\left[\chi^{\beta}, \chi^{\gamma}\right]}
\end{aligned}
$$

if fields are solutions of equations of motion of $S_{S Y M}$.

We see that on shell (on the space of solutions of the equations of motion where gauge equivalent solutions are identified) supersymmetry transformations commute with space-time translations:

$$
\left[\theta_{\alpha}, D_{i}\right]=0 \text { on shell. }
$$

Hence on shell $\theta_{\alpha}, D_{i}$ generate the standard supersymmetry algebra (this is wrong off shell). To reduce the theory to a point we should consider $A_{1}, \cdots A_{10}$ and $\chi^{1}, \cdots, \chi^{16}$ as constant matrices. 
We will introduce the Lie algebra $Y M$ in such a way that an $N$ - dimensional representation of the algebra $Y M$ gives a classical solution of the reduced SYM theory. More formally, we can define $Y M$ as a Lie algebra having even generators $\boldsymbol{D}_{1}, \ldots, \boldsymbol{D}_{10}$ and odd generators $\chi^{1}, \ldots, \chi^{16}$ obeying relations

$$
\begin{array}{r}
\sum_{i=1}^{10}\left[\boldsymbol{D}_{i},\left[\boldsymbol{D}_{i}, \boldsymbol{D}_{m}\right]\right]-\frac{1}{2} \sum_{\alpha \beta=1}^{16} \Gamma_{\alpha \beta}^{m}\left[\chi^{\alpha}, \boldsymbol{\chi}^{\beta}\right]=0 \quad m=1, \ldots, 10 \\
\sum_{\beta=1}^{16} \sum_{i=1}^{10} \Gamma_{\alpha \beta}^{i}\left[\boldsymbol{D}_{i}, \boldsymbol{\chi}^{\beta}\right]=0 \quad \alpha=1 \ldots 16
\end{array}
$$

Universal enveloping algebra $U(Y M)$ can be regarded as a unital associative algebra defined by the same relations as $Y M$.

The algebra $Y M$ is closely related to the graded Lie algebra $L=\sum L$ with generators $\boldsymbol{\theta}_{1}, \ldots, \boldsymbol{\theta}_{16}$ of degree one subject to relations

$$
\Gamma_{i_{1}, \ldots, i_{5}}^{\alpha \beta}\left[\boldsymbol{\theta}_{\alpha}, \boldsymbol{\theta}_{\beta}\right]=0
$$

Namely, one can prove that $Y M$ is isomorphic to $\bigoplus_{i \geq 2} L^{i} \subset L$ (the generator $\boldsymbol{D}_{m}$ is defined by the formula $\left[\boldsymbol{\theta}_{\alpha}, \boldsymbol{\theta}_{\beta}\right]=\Gamma_{\alpha \beta}^{i} \boldsymbol{D}_{i}$ and the generator $\boldsymbol{\chi}^{\alpha}$ by the formula $\Gamma_{\alpha \beta i} \boldsymbol{\chi}^{\beta}=\left[\boldsymbol{\theta}_{\alpha}, \boldsymbol{D}_{i}\right]$ ).

We can define also the Lie algebra $T Y M$ as follows

$$
T Y M \stackrel{\text { def }}{=} \bigoplus_{i \geq 3} L^{i} \subset L .
$$

This algebra is generated by as a Lie algebra (and its universal enveloping algebra $U(T Y M)$ as associative algebra) by expressions $\nabla_{i_{1}} \cdots \nabla_{i_{n}} \Phi$ where $\Phi$ is either $\boldsymbol{F}_{k l}$ or $\boldsymbol{\chi}^{\alpha}$ and $\nabla_{i}(x)=\left[\boldsymbol{D}_{i}, x\right]$ (we use the notation $\boldsymbol{F}_{i j}=\left[\boldsymbol{D}_{i}, \boldsymbol{D}_{j}\right]$ ). This means that an element of $U(T Y M)$ specifies a gauge-covariant local expression.

In the following by SUSY-deformations we understand deformations of the Lagrangian density and simultaneous deformation of the sixteen supersymmetries.

The SYM-theory has additional 16 trivial supersymmetries - the constant shifts of fermion fields. Analysis of deformations that preserves these symmetries was left out of scope of the present paper. 
As a first approximation to the problem we would like to solve we shall study infinitesimal supersymmetric deformations of equations of motion of tendimensional SUSY Yang-Mills theory. We translate this problem to a question in homological algebra. The homological reformulation leads to a highly nontrivial, but solvable problem. We shall analyze also super Poincaré invariant (= supersymmetric +Lorentz invariant) infinitesimal deformations. We shall prove that all of them are Lagrangian deformations of equations of motion (i.e. the deformed equations come from a deformed Lagrangian).

One of our tools is the theory of $\mathrm{A}_{\infty}$ and $\mathrm{L}_{\infty}$ algebras $\left[29\right.$. The theory of $\mathrm{L}_{\infty}$ algebras is closely related to $\mathrm{BV}$ formalism (see e.g. [42, 441). The theory of $\mathrm{L}_{\infty}$ algebras with invariant odd inner product is equivalent to classical BV-formalism if we are working at formal level [2]. (This means that we are considering all functions at hand as formal power series). The theory of $\mathrm{A}_{\infty}$ algebras arises if we would like to consider Yang-Mills theory for all gauge groups $\mathrm{U}(N)$ at the same time (see Appendix A).

Recall [41] that in classical BV-formalism the space of solutions to the equations of motion (EM) can be characterized as zero locus Sol of the odd vector field $Q$ that satisfies $[Q, Q]=0$. 2 It is convenient to work with the space $\mathrm{Sol} / \sim$ obtained from zero locus $\mathrm{Sol}$ after identification of physically equivalent solutions (see Sec. [6 for detail).

One can consider $Q$ as a derivation of the algebra of functionals on the space of fields $M$. One of the pieces of the input data an odd symplectic structure on the space $M$; vector field $Q$ preserves the odd symplectic form. The corresponding derivation can be written in the form $Q f=\{S, f\}$ where $\{\cdot, \cdot\}$ stands for the odd Poisson bracket and $S$ plays the role of the action functional in the BV formalism.

A vector field $q_{0}$ on $M$ is an infinitesimal symmetry of EM in BV-formalism if $\left[Q, q_{0}\right]=0$; we disregard trivial symmetries (symmetries of the form $q_{0}=$ $\left.\left[Q, q_{0}^{\prime}\right]\right)$. Let $f_{\tau_{1} \tau_{2}}^{\tau_{3}}$ be the structure constants of a Lie algebra $\mathfrak{g}$. The infinitesimal

\footnotetext{
${ }^{2}$ We use a unified notation $[\cdot, \cdot]$ for the commutators and super-commutators.
} 
symmetries $q_{\tau}$ in BV formalism define a $\mathfrak{g}$-action if

$$
\left[q_{\tau_{1}}, q_{\tau_{2}}\right]=f_{\tau_{1} \tau_{2}}^{\tau_{3}} q_{\tau_{3}}+\left[Q, q_{\tau_{1} \tau_{2}}\right]
$$

Here the index $\tau$ labels a basis in $\mathfrak{g}$. We say in this case that $\mathfrak{g}$ acts weakly on the space of fields. It is more convenient to work with $\mathrm{L}_{\infty}$ actions of $\mathfrak{g}$. To define an $\mathrm{L}_{\infty}$ action we complete the sequence of vector fields $q_{\tau}, q_{\tau_{1} \tau_{2}}$ by their higher analogs $q_{\tau_{1}, \ldots, \tau_{k}}, k=3, \ldots$ and impose relations generalizing (12). These relations are easy to write if we introduce a generating function $q=$ $\sum \frac{1}{k !} q_{\tau_{1}, \ldots, \tau_{k}} c^{\tau_{1}} \ldots c^{\tau_{k}}:$

$$
d_{\mathfrak{g}} q+[Q, q]+\frac{1}{2}[q, q]=0
$$

Here

$$
d_{\mathfrak{g}}=\frac{1}{2}(-1)^{\left|c^{\tau_{1}}\right|} f_{\tau_{2} \tau_{1}}^{\tau_{3}} c^{\tau_{1}} c^{\tau_{2}} \frac{\partial}{\partial c^{\tau_{3}}}
$$

stands for the differential calculating the Lie algebra cohomology of $\mathfrak{g}, c^{\tau}$ are the ghosts corresponding to the Lie algebra (cf. 24] where the sign of the operator is opposite to ours). Lagrangian BV version of Equation (13) contains Poisson bracket of functionals instead of the supercommutators of vector fields.

Deformations of a theory that preserve a Lie algebra of symmetries in BV language become deformations of a solution of equation (12). It is important to emphasize that we can start with an arbitrary BV formulation of the given theory and the classification of deformations does not depend on our choices. Classification of infinitesimal deformations is a homological problem. This problem concerns cohomology of the differential $d_{\mathfrak{g}}+[q, \cdot]$ that acts in the space of ghost-dependent vector fields.

In the present paper we apply such homological methods to the ten-dimensional SUSY Yang-Mills (SYM) theory and to its dimensional reductions. In particular, we describe all infinitesimal super Poincaré invariant deformations of tendimensional SYM theory and its reduction to a point. We show that almost all of them are given by a simple general formula. We analyze the extension of SUSY-invariant infinitesimal deformations to formal SUSY-invariant deformations. A formal deformation of a Lagrangian is a deformation that can be 
written as a formal power series with respect to some parameter; in string theory the role of this parameter is played by string tension $\alpha^{\prime}$. In the context of Yang-Mills theory we require that the Taylor coefficients of the series are derivatives of finite order of coefficients of the gauge potential and matter fields.

The paper will be organized in the following way: Preliminaries (Section 2) contains some mathematical information needed in our constructions and proofs. We do not use essentially the material of this section in sections 3 and 4Therefore one can skip this section at the first reading and start reading with Section 3 returning to Section 2 as necessary. In Section 3 we give a complete description of infinitesimal SUSY deformations of ten-dimensional SYM theory and its reduction to a point. We give a very explicit formula that works almost for all deformations. 3 In Section 7 we sketch the proof of the fact that infinitesimal SUSY deformations can be extended to formal deformations. In Section 4 we reduce the computation of the infinitesimal SUSY deformations to a homological problem. In Section 5 and in Appendix E we sketch the solution of this problem. In Section 6 we approach to the problem of infinitesimal deformations from the point of view of BV formalism. This approach leads to another homological formulation of our problem; this is the formulation sketched in the introduction.

In Appendix $\mathbb{C}$ we relate this formulation to formulation of Section 4 , The approach based on BV formalism works in more general situation than the formulation in Section 4 ,

The reader who is more interested in methods rather in concrete results can choose Appendices A, B as a starting point. These Appendices contain a brief exposition of the theory of $\mathrm{L}_{\infty}$ and $\mathrm{A}_{\infty}$ algebras and of the duality of differential associative algebras, that play an important role in our calculations.

In Appendix $\mathrm{H}$ we consider deformations of a $d$-dimensional reduction of ten-dimensional SYM theory when $0 \leq d \leq 10$. We show that many statements obtained for $d=0$ and $d=10$ can be generalized to any $d$.

\footnotetext{
${ }^{3}$ One can show that exceptional deformations are related to the homology of SUSY Lie algebra.
} 
The present paper concludes the series of papers devoted to the analysis of deformations of SYM theories [34, [35], [33, [36], 36]. It contains a review of most important results of these papers as well as some new constructions. The paper is mostly self-contained, but contain occasional references to the other papers in the series when it comes to proofs we intend to skip.

The supersymmetric deformations of maximally supersymmetric gauge theories were studied in numerous papers mostly in superspace approach or/and in pure spinor formalism (see, for example, [6, [12, [13, [14, [11] It is not always easy to compare the results of these papers with our results, but when this comparison is possible the results agree.

Acknowledgment Both authors would like to thank IHES (Bures-surIvette) and MPIM (Bonn) and the first author wishes to thank MPIM (Leipzig) for hospitality and excellent working conditions.

\section{Preliminaries}

\section{$2.1 \quad$ Basic algebras}

By now it became a common place (see e.g. [1, 25, ,4) that pure spinors can be used to formulate super Yang-Mills equations in dimension ten in manifestly supersymmetric manner. In this section we define objects relevant to such formulations.

The algebra $\mathcal{S}=\bigoplus_{k \geq 0} \mathcal{S}_{k}$ has generators

$$
\lambda^{1}, \ldots, \lambda^{16}
$$

and relations

$$
\Gamma_{\alpha \beta}^{i} \lambda^{\alpha} \lambda^{\beta}=0, i=1, \ldots, 10
$$

where $\Gamma_{\alpha \beta}^{i}$ are ten-dimensional $\Gamma$-matrices (see [20] for mathematical introduction). $\left(\lambda^{\alpha}\right)$ are coordinates on sixteen dimensional spinor representation in a basis $\left(\phi_{\alpha}\right)$. Any spinor $\phi$ is equal to $\lambda^{\alpha} \phi_{\alpha}$. Occasionally we shall identify a spinor $\psi$ in a basis-dependent fashion with an array of coordinates $\lambda=$ 
$\left(\lambda^{1}(\phi), \ldots, \lambda^{16}(\phi)\right) . \mathcal{S}$ is an algebra of polynomial functions on the space $\mathcal{C}$ of pure spinors (spinors obeying $\Gamma_{\alpha \beta}^{i} \lambda^{\alpha} \lambda^{\beta}=0$, cf. [15]). Components $\mathcal{S}_{k}$ of $\mathcal{S}$ are spaces of polynomial functions of degree $k$. Projectivization of $\mathcal{C} \backslash\{0\}$, i.e. result of identification of proportional pure spinors in $\mathcal{C} \backslash\{0\}$, is an Isotropic Grassmannian $\mathcal{Q}$ or, which is the same, projective space of pure spinors. The linear space $\mathcal{S}_{k}$ is the space of holomorphic sections of the line bundle $\mathcal{O}(k)$ over $\mathcal{Q}$ (see, for example, 23] for explanation of standard notations of algebraic geometry). The group $\operatorname{Spin}(10)$ acts on $\mathcal{S}$ in natural way; it is easy to check that $\mathcal{S}_{k}$ is an irreducible representation of $\operatorname{Spin}(10)$ with Dynkin label $[0,0,0, k, 0]$. Using this fact one can calculate the Poincaré series of $\mathcal{S}$ :

$$
\mathcal{S}(t)=\frac{1+5 t+5 t^{2}+t^{3}}{(1-t)^{11}}
$$

(see [7]).

The reduced Berkovits algebra $B_{0}$ is a differential graded commutative algebra. It is generated by even $\lambda^{\alpha}$ obeying pure spinor relations (16) and odd $\psi^{\alpha}$. The differential $d$ satisfies $d\left(\psi^{\alpha}\right)=\lambda^{\alpha}, d\left(\lambda^{\alpha}\right)=0$.

One can also give a description of $B_{0}$ in terms of functions on $\mathcal{C}$. Its elements are polynomial functions depending on pure spinor $\lambda$ and odd spinor $\psi$. We can interpret $\psi^{\alpha}$ as coordinates on odd spinor space $\Pi S$. The differential is represented by the odd vector field $\lambda^{\alpha} \frac{\partial}{\partial \psi^{\alpha}}$.

The (unreduced) Berkovits algebra $B$ can be defined as the algebra of polynomial functions of pure spinor $\lambda$, odd spinor $\psi$ and $x \in \mathbb{R}^{10}$. Sometimes it is convenient to modify this definition considering an algebra $B^{\infty}$ consisting of functions that are polynomial in $\lambda$ and $\psi$ but smooth as functions of $x \in \mathbb{R}^{10}$. The differential is defined as the derivation

$$
\lambda^{\alpha}\left(\frac{\partial}{\partial \psi^{\alpha}}+\Gamma_{\alpha \beta}^{i} \psi^{\beta} \frac{\partial}{\partial x^{i}}\right) .
$$

$\mathcal{S}, B_{0}, B$ are quadratic algebras, i.e. they are described by generators obeying quadratic relations. The reader is referred to [39] for a comprehensive account of quadratic algebras and references to the original publications. We shall be using construction of a quadratic dual algebra, which we remind the reader 
presently. Let $A=\bigoplus_{i \geq 0} A_{i}$ be a unital not necessarily commutative quadratic graded algebra $A$. We assume that $A_{0}=\mathbb{C}$. and that $A$ is generated by elements $w_{1}, \ldots, w_{n} \in A_{1}$ obeying quadratic relations $r_{k}=r_{k}^{i j} w_{i} w_{j}=0, k=$ $1, \ldots, l$. In more invariant terms we say that $A$ is generated by the linear span $W=<w_{1}, \ldots, w_{n}>=A_{1}$. Relations $\left(r_{k}\right)$, in turn, span a linear subspace $R \subset W \otimes W$. The quadratic dual $A^{!}=\bigoplus_{i \geq 0} A_{i}^{!}$is an algebra generated by dual linear space $W^{*}=\left\langle w^{* 1}, \ldots, w^{* n}\right\rangle$, where $\left\langle w_{i}, w^{* j}\right\rangle=\delta_{i}^{j}$. It relations are generating the subspace $R^{\perp} \subset W^{*} \otimes W^{*}$. In other words $R^{\perp}$ has a basis $s^{m}=\sum_{i j} s_{i j}^{m} w^{* i} w^{* j}, m=1, \ldots, n^{2}-l$. The matrices $s_{i j}^{m}$ form a basis in the space of solutions of the linear system $\sum_{i j} r_{k}^{i j} s_{i j}=0$ (when some of $w_{j}$ have different parities $\left|w_{j}\right|$ Koszul sign rule is in force: $\left.\sum_{i j}(-1)^{\left|w_{j}\right|\left|w^{* i}\right|} r_{k}^{i j} s_{i j}=0\right)$. In addition $A^{! !}=A$.

This duality preserves (graded) tensor product of algebras. The dual to polynomial algebra $\mathbb{C}[s]$ is the exterior algebra on one generator $\Lambda[\psi]$. A combination of these two facts gives an isomorphism $\mathbb{C}\left[s_{1}, \ldots, s_{k}\right]^{!} \cong \Lambda\left[\psi^{1}, \ldots, \psi^{k}\right]$.

Another interesting example is an algebra of polynomial functions on a quadric $A=\mathbb{C}\left[s_{1}, \ldots, s_{k}\right] / s_{1}^{2}+\cdots+s_{k}^{2}$. The dual algebra $A^{!}$is generated by $\psi^{1}, \ldots, \psi^{k}$ subject to relations $\psi^{i} \psi^{j}+\psi^{j} \psi^{i}=0$ and $\left(\psi^{i}\right)^{2}=\left(\psi^{j}\right)^{2}, i \neq j$. These are commutation relations in $\mathrm{D}=1, \mathrm{~N}=\mathrm{k}$ supersymmetry Lie algebra. The element

$$
H=\left(\psi^{i}\right)^{2}
$$

is central. The algebra $A^{!}$is closely related to the Clifford algebra $C l_{n}$.

Quadratic duality has some useful properties in case of Koszul algebras which we about to define. The tensor product $A_{1} \otimes A_{1}^{!}=W \otimes W^{*}$ is a subspace of $A \otimes A^{!}$. The tensor

$$
e=\sum_{i} w_{i} \otimes w^{* i} \in W \otimes W^{*}
$$

satisfies $e^{2}=0$ and defines a differential on any left $A \otimes A^{!}$-module $K$ :

$$
d(m)=e m, m \in K
$$

The module $K=A \otimes A^{!^{*}}$ contains a subspace $\mathbb{C}=A_{0} \otimes A_{0}^{!^{*}}$ which generates 
nontrivial subspace in cohomology $H\left(A \otimes A^{!^{*}}\right)$. Quadratic algebra $A$ is a Koszul algebra if this subspace exhausts the cohomology.

For example if $A=\mathbb{C}\left[s_{1}, \ldots, s_{k}\right]$, then $A \otimes A^{!^{*}}$ coincides with $\mathbb{C}\left[s_{1}, \ldots, s_{k}\right] \otimes$ $\Lambda\left[\xi_{1}, \ldots, \xi_{k}\right]$, equipped with the acyclic differential $s_{i} \frac{\partial}{\partial \xi_{i}}$. This is why the polynomial algebra is Koszul. If $A=\mathbb{C}\left[s_{1}, \ldots, s_{k}\right] / s_{1}^{2}+\cdots+s_{k}^{2}$, then $A^{!^{*}}$ coincides with $\Lambda\left[\xi_{1}, \ldots, \xi_{k}\right] \otimes \mathbb{C}[h]$, The generators $h$ is dual to (18). The differential $e$ in this case acts by

$$
s_{i} \frac{\partial}{\partial \xi_{i}}+s_{i} \xi_{i} \frac{\partial}{\partial h}
$$

The differential is acyclic and $\mathbb{C}\left[s_{1}, \ldots, s_{k}\right] / s_{1}^{2}+\cdots+s_{k}^{2}$ is Koszul. This differential is reminiscent of (17).

One of the properties of Koszul algebras is that $A$ is Koszul if and only if $A^{!}$is. The Poincaré series $A(t)=\sum_{i \geq 0} \operatorname{dim} A_{i} t^{i}$ and $A^{!}(t)=\sum_{i \geq 0} \operatorname{dim} A_{i}^{!} t^{i}$ of Koszul, quadratically dual algebras are related:

$$
A(t) A^{!}(-t)=1
$$

Idea to use Koszul duality for classification of deformations of SYM has been proven to be fruitful [36]. This is why we look at $\mathcal{S}, B_{0}, B$ from the perspective of quadratic duality. Most of the facts pertinent to this can be also found in 34] and 35.

An obvious starting point in this direction would be the calculation of quadratic duals. Algebras $B_{0}, B$ carry a differential. Quadratic duals in our setting will be algebras with a differential. At the initial stage of computation we simply ignore the differentials. After all the underlying algebras are found we take care of the differentials in dual pairs of objects. The differential in $B_{0}$ is linear. It defines a differential in $B_{0}^{!}$in obvious way. The differential in $B$ is more complicated- it contains linear and quadratic parts. The linear part defines a differential in $B^{!}$, the quadratic part defines a nontrivial commutator in $B^{!}$(see below). The algebras $\mathcal{S}^{!}, B_{0}^{!}$and $B^{!}$are dual to $\mathcal{S}, B_{0}$ and $B$ in the sense of Definition 28 (Appendix B).

The use of the negative grading indices in linear spaces is unavoidable in a systematic quadratic-duality theory. In particular if all gradings of an algebra 
are positive, the dual object has negative gradings. Negative indices from any other standpoints appear unnatural. Our attempt to reconcile these points of view is to use the following convention :

$$
N_{i}=N^{-i}
$$

The Koszul dual algebra to $\mathcal{S}$ is a graded algebra $U(L)$ on generators $\boldsymbol{\theta}_{1}, \ldots, \boldsymbol{\theta}_{16}$ of degree one subject to

$$
\Gamma_{i_{1}, \ldots, i_{5}}^{\alpha \beta}\left[\boldsymbol{\theta}_{\alpha}, \boldsymbol{\theta}_{\beta}\right]=0 .
$$

The element $e$ (see (19)) is $\lambda^{\alpha} \boldsymbol{\theta}_{\alpha}$. The algebra $U(L)$ is a universal enveloping algebra of the graded Lie algebra

$$
L=\sum_{n \geq 1} L^{n}
$$

that is defined by generators $\left(\boldsymbol{\theta}_{\alpha}\right)$ obeying the same relations (21). 4 (This is a particular case of general statement: the quadratic dual of commutative quadratic algebra is a universal enveloping algebra of Lie algebra.)

A basis $\left(\boldsymbol{\theta}_{\alpha}\right)$ in $L^{1}=S$ is dual to the basis $\left(\lambda^{\alpha}\right)$ (15). The Poincaré series

$$
U(L)(t)=\frac{(1+t)^{11}}{1-5 t+5 t^{2}-t^{3}}
$$

follows from (20). The second graded component $L^{2}$ of $L$ has dimension ten. This follows from the formula (23) for Poincaré series $U(L)(t)$. It is easy to see that one can find a basis $\boldsymbol{D}_{1}, \ldots, \boldsymbol{D}_{10}$ in $L^{2}$ obeying

$$
\left[\boldsymbol{\theta}_{\alpha}, \boldsymbol{\theta}_{\beta}\right]=\Gamma_{\alpha \beta}^{i} \boldsymbol{D}_{i}
$$

$\mathcal{S}^{!}$is significantly more complex than the Koszul dual to $\mathbb{C}\left[s_{1}, \ldots, s_{k}\right] / s_{1}^{2}+$ $\cdots+s_{k}^{2}$. Elements $\boldsymbol{D}_{i} \in L$ - the analogues of $H \in C l_{n}$ are not central.

The algebra $U(L)$ admits derivations $\frac{\partial}{\partial \theta_{\alpha}}$, that act by the formula

$$
\frac{\partial}{\partial \theta_{\alpha}} \theta_{\beta}=\delta_{\alpha}^{\beta}
$$

\footnotetext{
4 The reader should be warned that the currently used grading in $L$ is different from the grading of the general theory outlined in Appendix $B$ in the notations of Appendix $B$ the generators of $L$ have degree minus one and generators of $\mathcal{S}$ have degree two.
} 
and are compatible with the relations (21)

The dual algebra $B_{0}^{!}$to $B_{0}$ is a tensor product $U(L) \otimes \mathbb{C}\left[s_{1}, \ldots, s_{16}\right]$. The polynomial factor is dual to $\Lambda\left[\psi^{1}, \ldots, \psi^{16}\right]$. The adjoint to $\lambda^{\alpha} \frac{\partial}{\partial \psi^{\alpha}}$ acts on the generating set $\left\{\boldsymbol{\theta}_{\alpha}, s_{\alpha}\right\}$ in $B_{0}^{!}$by the formula

$$
\boldsymbol{\theta}_{\alpha} \rightarrow s_{\alpha}
$$

and defines a differential $d_{B_{0}^{!}}$in $B_{0}^{!}$. The algebra $B_{0}^{!}$is a universal enveloping of a Lie algebra $H$. It is a direct $\operatorname{sum} H=\bigoplus_{i \geq 0} H^{i}=L+S$, where $S=H^{0}=<s_{\alpha}>$ is an abelian Lie algebra in degree zero. The differential (26) has degree minus one in $H$. The duality in the sense of the definition 28 is established by means of the element $e=\lambda^{\alpha} \boldsymbol{\theta}_{\alpha}+\psi^{\alpha} s_{\alpha}$.

Let us introduce a Lie algebra $Y M$ on even generators $\boldsymbol{D}_{1}, \ldots, \boldsymbol{D}_{10}$ and odd $\chi^{1}, \ldots, \chi^{16}$ obeying relations

$$
\begin{aligned}
\sum_{i=1}^{10}\left[\boldsymbol{D}_{i},\left[\boldsymbol{D}_{i}, \boldsymbol{D}_{m}\right]\right]- & \\
& -\frac{1}{2} \sum_{\alpha \beta=1}^{16} \Gamma_{\alpha \beta}^{m}\left[\chi^{\alpha}, \chi^{\beta}\right]=0 \quad m=1, \ldots, 10
\end{aligned}
$$

$\sum_{\beta=1}^{16} \sum_{i=1}^{10} \Gamma_{\alpha \beta}^{i}\left[\boldsymbol{D}_{i}, \boldsymbol{\chi}^{\beta}\right]=0 \quad \alpha=1 \ldots 16$

The relations give a formal algebraic abstraction of equations of motion of $\mathrm{D}=10 \mathrm{SYM}$ theory reduced to a point. An $N$ - dimensional representations of the algebra $Y M$ gives a classical solution of the reduced SYM theory (of IKKT model ). It is easy to construct a homomorphism of the Lie algebra $Y M$ into $L$ (or, more precisely, into $\bigoplus_{k \geq 2} L^{k}$ ). Namely, we should send its generators into $\boldsymbol{D}_{i}$, defined by (24) and into $\chi^{\beta}$ defined by the formula

$$
\Gamma_{\alpha \beta i} \chi^{\beta}=\left[\boldsymbol{\theta}_{\alpha}, \boldsymbol{D}_{i}\right]
$$

It turns out that

$$
\boldsymbol{\theta}_{\alpha} \chi^{\boldsymbol{\beta}}=\Gamma_{\alpha}^{\beta i j}\left[\boldsymbol{A}_{i}, \boldsymbol{A}_{j}\right]
$$

in the Lie algebra $L$. 
Proposition 1 The algebra $Y M$ is isomorphic to $\bigoplus_{k \geq 2} L^{k}$. The obvious map $\bigoplus_{k \geq 2} L^{k} \rightarrow(L+S, d)$ is a quasi-isomorphism. Similarly $U(Y M)$ is quasiisomorphic to $B_{0}^{!}$.

Recall that a homomorphism of differential algebras (modules) is called a quasi-isomorphism if it induces an isomorphism in homology.

Let us denote by $\theta_{\alpha}$ the derivation of the algebra $Y M$ acting on generators by the formula (29|30) The derivations $\theta_{\alpha}$ can be interpreted as supersymmetry transformations. It is easy to check that they can be interpreted as commutators with generators of $L$ :

$$
\theta_{\alpha} x=\left[\boldsymbol{\theta}_{\alpha}, x\right]
$$

The dual to $B$ is the universal enveloping algebra of a graded semi-direct product $L \ltimes \Lambda$ of $L$ with abelian $\Lambda$. The generators $s_{1}, \ldots, s_{16}, \varsigma_{1}, \ldots, \varsigma_{10}$ of $\Lambda$ have degree zero and one respectively. The nontrivial commutation relations between $L$ and $\Lambda$ are

$$
\left[\boldsymbol{\theta}_{\alpha}, s_{\beta}\right]=\Gamma_{\alpha \beta}^{i} \varsigma_{i}
$$

The action of the nontrivial components of differential are given by the following formulas

$$
d\left(\boldsymbol{\theta}_{\alpha}\right)=s_{\alpha}, d\left(\boldsymbol{D}_{i}\right)=\varsigma_{i} .
$$

The duality is established by means of the element $e=\lambda^{\alpha} \boldsymbol{\theta}_{\alpha}+\psi^{\alpha} s_{\alpha}+x^{i} \varsigma_{i}$.

It is clear that $\boldsymbol{F}_{i j}=\left[\boldsymbol{D}_{i}, \boldsymbol{D}_{j}\right]$ and $\chi^{\alpha}$ belong to $T Y M \stackrel{\text { def }}{=} \bigoplus_{i \geq 3} L^{i} \subset L$. Moreover, they generate $T Y M$ as an ideal of $Y M$. More precisely, as an algebra $T Y M$ is generated by expressions $\nabla_{i_{1}} \cdots \nabla_{i_{n}} \Phi$ where $\Phi$ is either $\boldsymbol{F}_{k l}$ or $\chi^{\alpha}$ and $\nabla_{i}(x)=\left[\boldsymbol{D}_{i}, x\right]$. In the framework of ten dimensional Yang-Mills theory we can interpret these expressions as covariant derivatives of field strength and spinor field. Thus the elements of $U(T Y M)$ are algebraic abstractions of gauge covariant local expressions.

Proposition 2 Inclusion $T Y M \subset(\tilde{L}, d)$ is a quasi-isomorphism. Likewise $U(T Y M)$ and $B^{!}$is a pair of quasi-isomorphic algebras. 
One can prove that all quadratic algebras we use are Koszul algebras.

Notice $Y M$ and $T Y M$ are ideals in $L$, therefore elements $l \in L$ specify derivations $\alpha_{l}(x)=[l, x]$ on $Y M$ and $T Y M$. This allows us to realize elements of $L$ as vector fields on the space $S o l=S_{0} l_{N}$ of solutions of Yang-Mills equations with the gauge group $\mathrm{U}(N)$. (Notice that we do not identify gauge equivalent solutions.) More precisely, $L$ is realized is a Lie subalgebra of the LIe algebra of vector fields generated by supersymmetries. The universal enveloping algebras $U(T Y M), U(Y M)$ and $U(L)$ become associative subalgebras in algebras of differential operators Diff on the space of solutions.

\subsection{Calculation of the Lie algebra cohomology}

Computation of the Hochschild and the Lie algebra cohomology 5 is seldom done with the standard complexes. The reason is that the spaces of chains in these complexes are extremely big and redundant. In practical computations we are interested in more manageable complexes, that still have the same cohomology. Koszul duality theory provides us with very economic complexes. The appropriate constructions will be spelled out in this section.

Let us consider a graded commutative Koszul algebra $\mathcal{C}$ and its dual algebra $\mathcal{C}^{!}=\bigoplus_{i \geq 0} \mathcal{C}_{-i}^{!}=\bigoplus_{i \geq 0} \mathcal{C}^{! i}=U(\mathfrak{g})$ where $\mathfrak{g}$ is a graded Lie algebra. Let $N=$ $\bigoplus_{i \geq 0} N_{-i}=\bigoplus_{i \geq 0} N^{i}$ be a graded $\mathfrak{g}$-module (representation of $\mathfrak{g}$ ).

Proposition 3 [39] The cohomology $\mathrm{H}^{\bullet}(\mathfrak{g}, N)$ is equal to the cohomology of the complex $N_{c} \stackrel{\text { def }}{=} N \otimes \mathcal{C}$ (the $\mathcal{C}$-grading defines the cohomological grading in the tensor product). The subscript $c$ in $N_{c}$ stands for cohomology. The differential $d$ is defined by the left multiplication on

$$
e=w^{* i} \otimes w_{i} \in \mathcal{C}^{! 1} \otimes \mathcal{C}_{1} \subset \mathcal{C}^{!} \otimes \mathcal{C}
$$

\footnotetext{
${ }^{5}$ Definitions of Lie algebra cohomology and Hochschild cohomology are given in Appendix A. Appendix B contains a sketch of the proof of the results formulated in present section. The book [39] contains a detailed treatment of the material presented in this section. The book 44 is a modern elementary introduction to homological algebra that could be a good starting point for nonspecialists.
} 
The basis elements $w_{i} \in \mathcal{C}_{1}$ act on $\mathcal{C}$ by means of multiplication from the left and the action of the elements of the dual basis $w^{* i} \in\left(\mathcal{C}^{1}\right)^{*} \subset \mathcal{C}^{!}$is defined by means of representation of $\mathfrak{g}$ on $N$.

The subspaces $N_{c \boldsymbol{m}}^{\bullet}=\bigoplus_{i+j=\boldsymbol{m}} N_{j} \otimes \mathcal{C}_{i}$ are d-invariant 6 and $N_{c}^{\bullet}$ is the direct sum $\bigoplus_{m} N_{c m}^{\bullet}$ of subcomplexes.

The component $\mathrm{H}^{k, \boldsymbol{m}}(\mathfrak{g}, N)$ of $k$-th cohomology group of homogeneity $\boldsymbol{m}$ coincides with $\mathrm{H}^{k}\left(N_{c}^{\bullet}\right)$.

There exists a similar statement for Lie algebra homology. The complex $N_{h}^{\bullet}=$ $N \otimes \mathcal{C}^{*}$ is the direct sum of subcomplexes $N_{h}^{\bullet}=\bigoplus_{m} N_{h}^{\bullet}$. The homological grading on $N_{h}^{\bullet}$ is defined as follows:

$$
\begin{aligned}
& N_{h \boldsymbol{m}}^{\bullet}=\left(N_{m_{0}} \otimes \mathcal{C}_{m-m_{0}}^{*} \stackrel{d^{m_{0}}}{\rightarrow} \ldots N_{0} \otimes \mathcal{C}_{m}^{*} \stackrel{d^{0}}{\rightarrow} \ldots N_{m-1} \otimes \mathcal{C}_{1}^{*} \stackrel{d^{m-1}}{\rightarrow} N_{m} \otimes \mathcal{C}_{0}^{*}\right) \\
& \left(N \otimes \mathcal{C}^{*}\right)_{\boldsymbol{m}}=N_{m} \otimes \mathcal{C}_{0}^{*} \leftarrow N_{m-1} \otimes \mathcal{C}_{1}^{*} \leftarrow \cdots
\end{aligned}
$$

Proposition 4 [39] There is an isomorphism $\mathrm{H}_{\bullet}(\mathfrak{g}, N) \cong \mathrm{H}_{\bullet}\left(N \otimes \mathcal{C}^{*}\right)$ and its refinement

$$
\mathrm{H}_{k, \boldsymbol{m}}(\mathfrak{g}, N) \cong \mathrm{H}_{k}\left(\left(N \otimes \mathcal{C}^{*}\right)_{\boldsymbol{m}}\right)
$$

Let $\mathcal{C}$ be $\mathcal{S}, \mathcal{C}^{!}$be $U(L)$ and $N$ be an $L$-module. Propositions 344 give us alternative complexes for computation of $\mathrm{H}^{\bullet}(L, N), \mathrm{H}_{\bullet}(L, N)$.

Corollary 5 [39] The cohomology $\mathrm{H}^{\bullet}(L, N)$ is equal to the cohomology of the complexes $N_{c} \stackrel{\text { def }}{=} N \otimes \mathcal{S}$. The differential is a multiplication by

$$
e=\lambda^{\alpha} \boldsymbol{\theta}_{\alpha}
$$

The cohomological grading coincides with the grading of $\mathcal{S}$-factor. The total degree is preserved by $d$. The complex $N_{c}$ splits according to degree:

$$
\begin{aligned}
& N_{c m}^{\bullet}=\left(N_{m} \otimes \mathcal{S}_{0} \rightarrow N_{m+1} \otimes \mathcal{S}_{1} \rightarrow \ldots\right) \\
& (N \otimes \mathcal{S})^{m}=N^{m} \otimes \mathcal{S}_{0} \rightarrow N^{m+1} \otimes \mathcal{S}_{1} \rightarrow \cdots
\end{aligned}
$$

\footnotetext{
${ }^{6}$ To avoid a possible confusion of cohomological and internal homogeneous grading we reserve the bold index for the latter.
} 
The complex $N_{c \boldsymbol{m}}^{\bullet}$ is defined for positive and negative $\boldsymbol{m}$, we assume that $N_{m}=$ 0 if $m<m_{0}$. Then $\mathrm{H}^{k, \boldsymbol{m}}(L, N)=\mathrm{H}^{k}\left(N_{c}^{\bullet} \boldsymbol{m}\right)$.

There is also a degree decomposition in homology $\mathrm{H}_{k}(L, N)=\bigoplus_{\boldsymbol{m}} \mathrm{H}_{k, \boldsymbol{m}}(L, N)$.

Corollary 6 [39] The homology $\mathrm{H}_{\bullet}(L, N)$ is equal to the cohomology of the complex $N_{h} \stackrel{\text { def }}{=} N \otimes \mathcal{S}^{*}$. The space $\mathcal{S}^{*}=\bigoplus_{n \geq 0} \mathcal{S}_{n}^{*}$ is an $\mathcal{S}$-bimodule dual to $\mathcal{S}$. The differential is a multiplication by e (33). The homological degree coincides with the grading of $\mathcal{S}^{*}$-factor. The complex $N_{h}$ splits :

$$
\begin{aligned}
& N_{h \boldsymbol{m}}^{\bullet}=N_{m_{0}} \otimes \mathcal{S}_{m-m_{0}}^{*} \stackrel{d}{\rightarrow} \ldots N_{0} \otimes \mathcal{S}_{m}^{*} \stackrel{d}{\rightarrow} \ldots N_{m-1} \otimes \mathcal{S}_{1}^{*} \stackrel{d}{\rightarrow} N_{m} \otimes \mathcal{S}_{0}^{*} \\
& \text { and } \mathrm{H}_{k, \boldsymbol{m}}(L, N)=\mathrm{H}^{m-k}\left(N_{h \boldsymbol{m}}^{\bullet}\right) \text {. }
\end{aligned}
$$

The Propositions 213 are particular cases of more general statements formulated in terms of Hochschild cohomology and homology (see Appendix B, Propositions 30[31)

\subsection{The group $\operatorname{Spin}(10, \mathbb{C})$ and the space of pure spinors}

The complex group $\operatorname{Spin}(10, \mathbb{C})$ acts transitively on the projective space of pure spinors $\mathcal{Q}$; the stabilizer of a point is a parabolic subgroup and parabolic subgroup corresponding to different points are conjugated. To describe the Lie algebra $\mathfrak{p}$ of one of such parabolic subgroups $P$ we notice that the Lie algebra $\mathfrak{s o}(10, \mathbb{C})$ of $\mathrm{SO}(10, \mathbb{C})$ can be identified with $\Lambda^{2} V$. This is the same as a the space of antisymmetric tensors $\rho_{a b}$ where $a, b=1, \ldots, 10$, if a basis $\left(v_{1}, \ldots, v_{10}\right)$ in $V$ is fixed. The vector representation $V$ of $\mathrm{SO}(10, \mathbb{C})$ splits upon restriction to $\mathrm{GL}(5, \mathbb{C}) \subset \mathrm{SO}(10, \mathbb{C})$ into the direct sum $W+W^{\prime}$ of vector and representation $W^{\prime}$ isomorphic to covector representations $W^{*}$. We shall not make a distinction between $W^{\prime}$ and $W^{*}$. The Lie algebra of $\mathrm{SO}(10, \mathbb{C})$ can be decomposed into a sum $\Lambda^{2} W+\mathfrak{p}$ of vector spaces. Subspace $\mathfrak{p}=W \otimes W^{*}+\Lambda^{2} W^{*}$ is the Lie algebra of $P$. Suppose that $\left(v_{1}, \ldots, v_{5}\right)$ is a basis $W$ and $\left(v_{6}, \ldots, v_{10}\right)$ in $W^{*}$. Using the language of generators we can say that the Lie algebra $\mathfrak{s o}(10, \mathbb{C})$ is generated 
by skew-symmetric tensors $m_{a b}, n^{a b}$ and by $k_{a}^{b}$ where $a, b=1, \ldots, 5$. These are blocks of $\rho_{a b}$ defined by the chosen partition of the basis. The subalgebra $\mathfrak{p}$ is generated by $k_{a}^{b}$ and $n^{a b}$. Corresponding commutation relations are

$$
\begin{aligned}
& {\left[m, m^{\prime}\right]=\left[n, n^{\prime}\right]=0} \\
& {[m, n]_{a}^{b}=m_{a c} n^{c b}} \\
& {[m, k]_{a b}=m_{a c} k_{b}^{c}+m_{c b} k_{a}^{c}} \\
& {[n, k]_{a b}=n^{a c} k_{c}^{b}+n^{c b} k_{c}^{a}}
\end{aligned}
$$

Homomorphism $\operatorname{Spin}(10, \mathbb{C}) \rightarrow \mathrm{SO}(10)$ defines cover in two sheets. Let $\widetilde{P}$ be the preimage of $P$ in $\operatorname{Spin}(10, \mathbb{C})$ under this projection. The group $\widetilde{P}$ contains a double sheeted cover $\widetilde{\mathrm{GL}}(5, \mathbb{C})$ of $\mathrm{GL}(5, \mathbb{C})$. There exists one-to-one correspondence between $\operatorname{Spin}(10, \mathbb{C})$-homogeneous holomorphic vector bundles over $\mathcal{Q}$ and complex representations of $\widetilde{P}$ (see e.g. [8]) in the fiber over the $P$-fixed point. A one-dimensional representation of $\widetilde{P}$ corresponding to the line bundle $\mathcal{O}(k)$ over $\mathcal{Q}$ will be denoted $\mu_{k}$. In this representation $g \in \widetilde{\mathrm{GL}}(5, \mathbb{C})$ acts by multiplication on $\sqrt{\operatorname{det}}^{k}(g)$.

The space of spinors can be embedded into Fock space $\mathcal{F}$ (see [15] for mathematical account). This Fock space is a representation of canonical anti-commutation relations $a_{i} a_{j}+a_{j} a_{i}=0, a_{i}^{*} a_{j}^{*}+a_{j}^{*} a_{i}^{*}=0, a_{i} a_{j}^{*}+a_{j}^{*} a_{i}=\delta_{i j} i, j=1, \ldots, 5$. The cone $\mathcal{C} \backslash\{0\}$ can be realized as the orbit of Fock vacuum with respect to the action of the group of linear canonical transformations (transformations preserving anti-commutation relations), that preserve chirality. For every vector $x \in \mathcal{F}$ we consider the subspace $W^{*}(x)$ of the space $V$ of linear combinations $A=\sum \rho^{i} a_{i}+\sum \tau^{j} a_{j}^{*}$ obeying $A x=0$. For $x \in \mathcal{Q}$ the subspace $W^{*}(x)$ is fivedimensional. The subspaces $W^{*}(x)$ specify a $\operatorname{Spin}(10)$-invariant vector bundle over $\mathcal{Q}$ that will be denoted by $\mathcal{W}^{*}$; corresponding representation of $P$ will be denoted by $W^{*}$. The bundle over $\mathcal{Q}$ having fibers $V / W^{*}(x)$ will be denoted by $\mathcal{W}$; corresponding representation of $P$ will be denoted by $W$. 
Notice that $\operatorname{Spin}(10)$-representation contents of first two components of $L$ is

$$
\begin{aligned}
& L^{1}=[0,0,0,1,0] \\
& L^{2}=[1,0,0,0,0] \\
& L^{3}=[0,0,0,0,1] \\
& L^{4}=[0,1,0,0,0] \\
& \ldots
\end{aligned}
$$

And this is how they split as $\widetilde{\mathrm{GL}}(5)$-representations :

$$
\begin{aligned}
& L^{1}=\mu_{-1}+\Lambda^{2}(W) \otimes \mu_{-1}+\Lambda^{4}(W) \otimes \mu_{-1} \\
& L^{2}=W^{*}+W \\
& L^{3}=\Lambda^{4}\left(W^{*}\right) \otimes \mu_{1}+\Lambda^{2}\left(W^{*}\right) \otimes \mu_{1}+\mu_{1} \cong \\
& \cong W \otimes \mu_{-1}+\Lambda^{3}(W) \otimes \mu_{-1}+\mu_{1} \\
& L^{4}=\Lambda^{2}(W)+\Lambda^{2}\left(W^{*}\right)+W \otimes W^{*} \\
& \ldots
\end{aligned}
$$

The above formulas are written in such a way that the leftmost summand in every line is a representation of $\widetilde{P}$; the same is true for the sum of first two leftmost summands.

\section{$2.4 \quad$ Euler characteristics}

Statements formulated in Section 2.2 permit us to calculate the Euler characteristics of $\mathrm{H}^{\bullet}(L, N)$ and $\mathrm{H}_{\bullet}(L, N)$.

Suppose a complex of vector spaces $K=\sum K^{k}$ has finite number of finitedimensional cohomology groups $\mathrm{H}=\sum \mathrm{H}^{k}$. Then the Euler characteristic can be defined as $\chi=\chi(\mathrm{H})=\sum(-1)^{k} \operatorname{dim} \mathrm{H}^{k}$. If $K$ has finite number of finitedimensional components $K^{k}$ then

$$
\chi(\mathrm{H})=\sum(-1)^{k} d_{k}
$$

where $d_{k}=\operatorname{dim} K^{k}$. 
A Lie group $G$ action on complex $K^{i}$ by operators $T_{K^{i}}(g), g \in G$ descends to cohomology. The Euler characteristic $\chi_{K}(g)$ is defined as the alternating sum of characters $\sum(-1)^{k} \operatorname{tr} T_{\mathrm{H}^{k}}(g)$. The relation (47) remains correct in this more general situation after appropriate modifications, namely $d_{k}$ gets replaced by $\operatorname{tr} T_{K^{i}}(g) 7$

A bit of terminology: if a group $G$ acts on a graded vector space $A=$ $\bigoplus_{i \geq n_{0}} A_{i}$ by linear automorphisms $T_{A_{i}}(g)$ then $G$-equivariant Poincaré series is defined as a generating function of characters:

$$
A(g, t)=\sum_{i \geq n_{0}} \operatorname{tr} T_{A_{i}}(g) t^{i}
$$

(In the case of trivial group action this definition is equivalent to the standard definition: $A(t)=\sum_{i \geq n_{0}} \operatorname{dim} A_{i} t^{i}$.)

In our applications module $N$ over Lie algebra $L$ is a graded $\operatorname{Spin}(10)$ representation. The $\operatorname{Spin}(10)$-action on $N$ is compatible with the action on $L$. We will give an expression of $\chi_{\mathrm{H}^{\bullet}(L, N)}(g, t)$ and of $\chi_{\mathrm{H}_{\bullet}(L, N)}(g, t)$, in terms of Poincaré series $N(g, t), \mathcal{S}(g, t)$.

Let us sketch the derivation in more general case when $N$ is a graded module over graded Lie algebra $\mathfrak{g}$ and a group $K$ acts on $\mathfrak{g}$ and $N$ in compatible way. (The Poincaré series of $\mathfrak{g}$ and $N$ and Euler characteristic of $\mathrm{H}^{k}(\mathfrak{g}, N)$ depend on $g \in K$ and on $t$.)

The Lie algebra cohomology $\mathrm{H}^{k}(\mathfrak{g}, N)$ coincides with Hochschild cohomology $\mathrm{H}^{k}(U(\mathfrak{g}), N)$ and therefore can be computed with the normalized Hochschild complex $C^{k}(U(g), N)=\operatorname{Hom}\left(I^{\otimes k}, N\right)$ where $I$ is the set of elements of the universal enveloping $U(\mathfrak{g})$ without the constant term. Formally we can express the Euler characteristic in terms of Poincaré series for $N$ and $U(\mathfrak{g})$ :

$$
\chi_{\mathrm{H} \bullet(\mathfrak{g}, N)}=\frac{N(g, t)}{U(\mathfrak{g})\left(g^{-1}, t^{-1}\right)}
$$

In the derivation of this relation we use the formula

$$
\frac{1}{u}=\frac{1}{1+(u-1)}=\sum(-1)^{k}(u-1)^{k} .
$$

\footnotetext{
${ }^{7}$ Using the notion of virtual representations ( see Appendix I) we can define the Euler characteristic of graded representation $K=\sum K^{i}$ as an alternating sum $\chi_{K}=\sum(-1)^{i} K^{i}$.
} 
Likewise homology $\mathrm{H}_{k}(\mathfrak{g}, N)$ can be computed with the normalized Hochschild complex $C_{k}(U(g), N)=I^{\otimes k} \otimes N$ and

$$
\chi_{\mathrm{H} \cdot(\mathfrak{g}, N)}=\frac{N(g, t)}{U(\mathfrak{g})(g, t)}
$$

For us this means that

$$
\begin{aligned}
\chi_{\mathrm{H}^{\bullet}(L, N)}(g, t) & =\frac{N(g, t)}{U(L)\left(g^{-1}, t^{-1}\right)}, \\
\chi_{\mathrm{H}_{\bullet}(L, N)}(g, t) & =\frac{N(g, t)}{U(L)(g, t)} .
\end{aligned}
$$

The expression for the Poincaré series $U(L)(g, t)$ will be derived at the end of this section.

We have mentioned already that the $n$-th graded component of $\mathcal{S}$ is an irreducible representation of $\operatorname{Spin}(10)$ with Dynkin label $[0,0,0, n, 0]$. Using this fact one can express $\mathcal{S}(g, t)$ in terms of the character $V(g)$ of vector representation $V$ and the character $S(g)$ of the spinor representation $S$ labelled by $[0,0,0,1,0]$. The character of the algebra $\mathcal{S}$ is given by the formula

$$
\mathcal{S}(g, t)=\left(1-V(g) t^{2}+S\left(g^{-1}\right) t^{3}-S(g) t^{5}+V(g) t^{6}-t^{8}\right) \operatorname{Sym} S(g, t) .
$$

There are several ways to prove this important formula. The homological approach of [19] is based on construction of a resolution of $\mathcal{S}$ as SymS-module. Another way of tackling the problem is to use fixed point formula [7. Standard monomial theory (see [37 ) can be utilized to arrive at the same result. The information about $\mathcal{S}$ permits us to analyze the structure of Koszul dual algebra $U(L)$ using the following general statement.

Proposition 7 Let $A$ be a Koszul algebra, equipped with an action of a group $G$. Then the group $G$ also acts on $A^{!}$and there is an equality

$$
A(g, t) A^{!}\left(g^{-1},-t\right)=1
$$

Proof. It is a trivial adaptation of the proof [40] to the case of algebra with $G$-action. The complex $A \otimes\left(A^{!}\right)^{*}$ has trivial cohomology by the definition of Koszul algebra. It decomposes into a direct sum of acyclic complexes $K_{n}=$ 
$\bigoplus_{i+j=n} A_{i} \otimes\left(A_{j}^{!}\right)^{*}$. The generating function of Euler characteristics of $K_{n}$ is equal to the constant function 1. But it also equals to the product of the generating functions $A(g, t) A^{!}\left(g^{-1},-t\right)$. (We use here the fact that the character of the dual representation is expressed in terms of the character $\rho(g)$ of original representation as $\rho\left(g^{-1}\right)$.)

Note that $V(g)=V\left(g^{-1}\right)$, because the ten-dimensional tautological representation of $\mathrm{SO}(10)$ is self-dual. For spinors we have $S^{*}(g)=S\left(g^{-1}\right)$.

Corollary 8 The Poincaré series $U(L)(t, g)$ of the universal enveloping $U(L)$ is equal to

$$
U(L)(g, t)=\frac{\Lambda S^{*}(g, t)}{1-V\left(g^{-1}\right) t^{2}-S\left(g^{-1}\right) t^{3}+S(g) t^{5}+V(g) t^{6}-t^{8}}
$$

Proof. Apply Proposition 7 to algebras $\mathcal{S}, \mathcal{S}^{!} \cong U(L)$ and $\operatorname{Sym}(S)^{!} \cong \Lambda\left(S^{*}\right)$.

Notice that instead of working with characters we could work with virtual representations and representation rings (see Appendix I). Then the above formula looks nicer:

$$
U(L)=\frac{\lambda_{t}\left(S^{*}\right)}{1-V t^{2}-S^{*} t^{3}+S t^{5}+V t^{6}-t^{8}}
$$

\section{Infinitesimal SUSY Deformations of $\mathcal{L}_{S Y M}$}

Let us consider an infinitesimal deformation $\delta \mathcal{L}$ of a Lagrangian $\mathcal{L}$. If an infinitesimal deformation $\delta^{\prime} \mathcal{L}$ is obtained from $\delta \mathcal{L}$ by means of a field redefinition then the action functionals corresponding to infinitesimal deformations $\delta \mathcal{L}, \delta^{\prime} \mathcal{L}$ coincide on solutions of EM for $\mathcal{L}$. The converse statement is also true. Therefore we will identify infinitesimal deformations $\delta \mathcal{L}$ and $\delta^{\prime} \mathcal{L}$ of $\mathcal{L}_{S Y M}$ if $\delta^{\prime} \mathcal{L}=\delta \mathcal{L}$ on the solutions of EM for $\mathcal{L}_{S Y M} .8$

\footnotetext{
${ }^{8}$ To reach a better understanding of the above statements we will discuss a finitedimensional analogy.

Any function

$$
f: \mathbb{C}^{n} \rightarrow \mathbb{C}
$$

can be deformed by adding an arbitrary function $g$ multiplied by an infinitesimal parameter. It is not true however that the space of deformations of $f$ coincides with the space $\hat{\mathcal{O}}$ of all $g$.
} 
We will be interested in deformations of SYM that are defined simultaneously for all gauge groups $\mathrm{U}(N)$. Let us consider first the Lagrangian $\mathcal{L}_{S Y M}$ reduced to a point. The deformation of the kind we are interested in are singletrace deformations: they can be represented in the form $\operatorname{tr} \Lambda$, where $\Lambda$ is an arbitrary non-commutative polynomial in terms of the fields of the reduced theory. The fields form an array of $N \times N$ matrix variables $A_{1}, \ldots, A_{10}, \chi^{1}, \ldots, \chi^{16}$ of suitable parity in the theory with the gauge group $\mathrm{U}(N)$. Reality conditions are left out of scope of our analysis, and we simply consider fields as complex matrices, i.e. as elements of $\mathrm{Mat}_{N}$. ( This means that the gauge group $\mathrm{U}(N)$ is extended to its complexification $G L(N)$.) We are working with all of these groups simultaneously, hence we consider the fields as formal non-commuting variables, i.e. as generators of free graded associative algebra . More precisely, we consider free graded associative algebra $\mathcal{A}$ generated by symbols of fields

$$
D_{i}, \chi^{\alpha}
$$

The space of single-trace deformations can be identified with $\mathcal{A} /[\mathcal{A}, \mathcal{A}] .9(\mathrm{~A}$ non-commutative polynomial of matrix variables $A_{1}, \ldots, A_{10}, \chi^{1}, \ldots, \chi^{16}$ can be considered as an element of $\mathcal{A}$. However, the (super)trace of (super) commutator vanishes, therefore the trace of this polynomial can regarded as an element of $\mathcal{A} /[\mathcal{A}, \mathcal{A}]$.) The natural map $\mathcal{A} \rightarrow \mathcal{A} /[\mathcal{A}, \mathcal{A}]$ will be denoted by tr .) However, we should take into account that the deformations can be equivalent (related by a

The reason is that there are trivial deformations of $f$ obtained by a change of parametrization of $\mathbb{C}^{n}$. A vector field $\xi$ on $\mathbb{C}^{n}$ defines an infinitesimal change of coordinates, under which $f$ transforms to $f_{\xi}=\xi^{i} \frac{\partial f}{\partial x i}$. The space $V$ ect $f$ of functions $f_{\xi}$ forms a subspace of trivial infinitesimal deformations. The quotient $\hat{\mathcal{O}} / V$ ect $f$ is the formal tangent space to the space of nontrivial deformations of (51).

Under some conditions of regularity one can identify $\hat{\mathcal{O}} / V$ ect $f$ with the algebra of functions on the set of critical points of $f$. (If this set is considered as a scheme the conditions of regularity are not necessary.)

In field theories this identification corresponds to identification of off-shell classes of deformations of an action functional with the deformations considered on shell (on the solutions of EM).

9 We can identify this space also with the space of cyclic words in the alphabet where letters correspond to the fields. 
change of variables). As we have seen this means that the deformations coincide on shell. In other words, the space of equivalence classes of deformations can be identified with $U(Y M) /[U(Y M), U(Y M)]$ where $U(Y M)$ can be interpreted as an associative algebra generated by the fields of SYM theory reduced to a point with relations coming from the equations of motion (see Section 1 and Section 2 for more detail). 10

Similar results are true for non-reduced SYM theory. In this case we consider the Lagrangian as a gauge invariant local expression (a trace of gauge covariant local expression). We are working with all groups $\mathrm{U}(N)$ simultaneously, hence we are writing a Lagrangian in the form $\operatorname{tr} H$ where $H \in \mathrm{U}(T Y M)$ and $\operatorname{tr}$ stand for the natural homomorphism $\operatorname{tr}: \mathrm{U}(T Y M) \rightarrow[\mathrm{U}(T Y M), \mathrm{U}(T Y M)]$. Notice, that in this case the Lagrangian is defined up to a total derivative 11

The supersymmetry transformations $\theta_{\alpha}$ act in natural way on all spaces we have considered.

We are saying that infinitesimal deformation $\delta \mathcal{L}$ of reduced SYM theory is supersymmetric if $\theta_{\alpha} \delta \mathcal{L}$ vanishes on equations of motion of $\mathcal{L}_{S Y M}$. For nonreduced theory a deformation is supersymmetric if $\theta_{\alpha} \delta \mathcal{L}$ is a total derivative on equations of motion of $\mathcal{L}_{S Y M}$.

Poincaré invariance is defined in a similar way.

There exists an infinite number of infinitesimal super Poincaré invariant deformations. Most of them are given by a simple general formula below, but for the non-reduced theory there are three exceptional deformations which do not fit into this formula. The first was discussed earlier in [3].

\footnotetext{
${ }^{10}$ This space has also interpretation in terms of Hochschild homology $\mathrm{H}_{0}(U(Y M), U(Y M))$ or in terms of cyclic homology.

${ }^{11}$ We say that a function on $\mathbb{R}^{n}$ is a total derivative if it can be represented in the form $\frac{\partial}{\partial x^{i}} H^{i}$. In more invariant way one can say that the differential form of degree $n$ corresponding to this function should be exact. Saying that Lagrangian is defined up to a total derivative we have in mind that adding $\frac{\partial}{\partial x^{i}} H^{i}$ where $\mathrm{H}^{i}$ are gauge invariant local expressions we obtain an equivalent Lagrangian.
} 


$$
\begin{aligned}
& \delta \mathcal{L}_{16}(\nabla, \chi)=\operatorname{tr}\left(\frac{1}{8} F_{m n} F_{n r} F_{r s} F_{s m}-\frac{1}{32}\left(F_{m n} F_{m n}\right)^{2}\right. \\
& +i \frac{1}{4} \chi^{\alpha} \Gamma_{m \alpha \beta}\left(\nabla_{n} \chi^{\beta}\right) F_{m r} F_{r n} \\
& -i \frac{1}{8} \chi^{\alpha} \Gamma_{m n r \alpha \beta}\left(\nabla_{s} \chi^{\beta}\right) F^{m n} F_{r s} \\
& +\frac{1}{8} \chi^{\alpha} \Gamma_{\alpha \beta}^{m}\left(\nabla_{n} \chi^{\beta}\right) \chi^{\Gamma} \Gamma_{m \Gamma \delta}\left(\nabla_{n} \chi^{\delta}\right) \\
& \left.-\frac{1}{4} \chi^{\alpha} \Gamma_{\alpha \beta}^{m}\left(\nabla_{n} \chi^{\beta}\right) \chi^{\gamma} \Gamma_{n \gamma \delta}\left(\nabla_{m} \chi^{\delta}\right)\right)
\end{aligned}
$$

It is convenient to introduce a grading on the space of fields (2) . We suppose that grading is multiplicative and $\operatorname{deg}\left(\nabla_{i}\right)=2, \operatorname{deg}\left(\chi^{\alpha}\right)=3$. This grading is related to the grading with respect to $\alpha^{\prime}$, that comes from string theory, by the formula

$$
\operatorname{deg}_{\alpha^{\prime}}=\frac{\operatorname{deg}-8}{4} .
$$

Subscript in $\delta \mathcal{L}_{16}$ in the formula (53) stands for the grading of infinitesimal Lagrangian. Lagrangian $\delta \mathcal{L}_{16}$ is a super Poincaré invariant deformation of lowest possible degree 12 The next linearly independent infinitesimal super Poincaré invariant deformation (of degree 20) was found in [16]. It has the following Lagrangian

$$
\begin{aligned}
\delta \mathcal{L}_{20}(\nabla, \chi)=f^{X Y Z} & f^{V W Z}\left[2 F_{a b}{ }^{X} F_{c d}{ }^{W} \nabla_{e} F_{b c}{ }^{V} \nabla_{e} F_{a d}{ }^{Y}-2 F_{a b}{ }^{X} F_{a c}{ }^{W} \nabla_{d} F_{b e}{ }^{V} \nabla_{d} F_{c e}{ }^{Y}\right. \\
& +F_{a b}{ }^{X} F_{c d}{ }^{W} \nabla_{e} F_{a b}{ }^{V} \nabla_{e} F_{c d}{ }^{Y} \\
& -4 F_{a b}{ }^{W} \nabla_{c} F_{b d}{ }^{Y} \chi^{\alpha X} \Gamma_{\alpha \beta a} \nabla_{d} \nabla_{c} \chi^{\beta V}-4 F_{a b}{ }^{W} \nabla_{c} F_{b d}{ }^{Y} \chi^{\alpha X} \Gamma_{\alpha \beta d} \nabla_{a} \nabla_{c} \chi^{\beta V} \\
& \left.+2 F_{a b}{ }^{W} \nabla_{c} F_{d e}{ }^{Y} \chi^{\alpha X} \Gamma_{\alpha \beta a d e} \nabla_{b} \nabla_{c} \chi^{\beta V}+2 F_{a b}{ }^{W} \nabla_{c} F_{d e}{ }^{Y} \chi^{\alpha X} \Gamma_{\alpha \beta a b d} \nabla_{e} \nabla_{c} \chi^{\beta V}\right]+
\end{aligned}
$$

\footnotetext{
${ }^{12}$ We will treat the truly lowest order deformation $\delta \mathcal{L}=\mathcal{L}_{S Y M}$ as trivial.
} 


$$
\begin{aligned}
& +f^{X Y Z} f^{U V W} f^{T U X}\left[4 F_{a b}^{Y} F_{c d}^{Z} F_{a c}{ }^{V} F_{b e}{ }^{W} F_{d e}{ }^{T}+2 F_{a b}{ }^{Y} F_{c d}{ }^{Z} F_{a b}{ }^{V} F_{c e}{ }^{W} F_{d e}{ }^{T}\right. \\
& -11 F_{a b}{ }^{Y} F_{c d}{ }^{Z} F_{c d}{ }^{V} \chi^{\alpha T} \Gamma_{\alpha \beta a} \nabla_{b} \chi^{\beta W}+22 F_{a b}{ }^{Y} F_{c d}{ }^{Z} F_{a c}{ }^{V} \chi^{\alpha T} \Gamma_{\alpha \beta b} \nabla_{d} \chi^{\beta W} \\
& +18 F_{a b}{ }^{Y} F_{c d}{ }^{V} F_{a c}{ }^{W} \chi^{\alpha T} \Gamma_{\alpha \beta b} \nabla_{d} \chi^{\beta Z}+12 F_{a b}{ }^{T} F_{c d}{ }^{Y} F_{a c}{ }^{V} \chi^{\alpha Z} \Gamma_{\alpha \beta b} \nabla_{d} \chi^{\beta W} \\
& +28 F_{a b}^{T} F_{c d}{ }^{Y} F_{a c}{ }^{V} \chi^{\alpha W} \Gamma_{\alpha \beta b} \nabla_{d} \chi^{\beta Z}-24 F_{a b}{ }^{Y} F_{c d}{ }^{V} F_{a c}{ }^{T} \chi^{\alpha W} \Gamma_{\alpha \beta b} \nabla_{d} \chi^{\beta Z} \\
& +8 F_{a b}{ }^{T} F_{c d}{ }^{Y} F_{a c}{ }^{Z} \chi^{\alpha V} \Gamma_{\alpha \beta b} \nabla_{d} \chi^{\beta W}-12 F_{a b}{ }^{T} F_{a c}{ }^{Y} \nabla_{b} F_{c d}{ }^{V} \chi^{\alpha Z} \Gamma_{\alpha \beta d} \chi^{\beta W} \\
& -8 F_{a b}{ }^{Y} F_{a c}{ }^{T} \nabla_{b} F_{c d}{ }^{V} \chi^{\alpha Z} \Gamma_{\alpha \beta d} \chi^{\beta W}+22 F_{a b}{ }^{V} F_{a c}{ }^{Y} \nabla_{b} F_{c d}{ }^{T} \chi^{\alpha Z} \Gamma_{\alpha \beta d} \chi^{\beta W} \\
& -4 F_{a b}{ }^{Y} F_{c d}{ }^{T} \nabla_{e} F_{a c}{ }^{V} \chi^{\alpha Z} \Gamma_{\alpha \beta b d e} \chi^{\beta W}+4 F_{a b}{ }^{Y} F_{a c}{ }^{T} \nabla_{c} F_{d e}{ }^{V} \chi^{\alpha Z} \Gamma_{\alpha \beta b d e} \chi^{\beta W} \\
& +4 F_{a b}{ }^{T} F_{c d}{ }^{Y} F_{c e}{ }^{V} \chi^{\alpha Z} \Gamma_{\alpha \beta a b d} \nabla_{e} \chi^{\beta W}-8 F_{a b}{ }^{Y} F_{c d}{ }^{T} F_{c e}{ }^{V} \chi^{\alpha Z} \Gamma_{\alpha \beta a b d} \nabla_{e} \chi^{\beta W} \\
& +6 F_{a b}{ }^{V} F_{c d}{ }^{Y} F_{c e}{ }^{W} \chi^{\alpha Z} \Gamma_{\alpha \beta a b d} \nabla_{e} \chi^{\beta T}+5 F_{a b}{ }^{V} F_{c d}{ }^{W} F_{c e}{ }^{Y} \chi^{\alpha Z} \Gamma_{\alpha \beta a b d} \nabla_{e} \chi^{\beta T} \\
& +6 F_{a b}{ }^{Y} F_{a c}{ }^{T} F_{d e}{ }^{V} \chi^{\alpha Z} \Gamma_{\alpha \beta b c d} \nabla_{e} \chi^{\beta W}-2 F_{a b}{ }^{Y} F_{a c}{ }^{T} F_{d e}{ }^{Z} \chi^{\alpha V} \Gamma_{\alpha \beta b c d} \nabla_{e} \chi^{\beta W} \\
& +4 F_{a b}{ }^{Y} F_{a c}{ }^{V} F_{d e}{ }^{Z} \chi^{\alpha W} \Gamma_{\alpha \beta b c d} \nabla_{e} \chi^{\beta T}+4 F_{a b}{ }^{T} F_{c d}{ }^{V} F_{c e}{ }^{Y} \chi^{\alpha Z} \Gamma_{\alpha \beta a b d} \nabla_{e} \chi^{\beta W} \\
& -4 F_{a b}{ }^{Y} F_{c d}{ }^{V} F_{c e}{ }^{W} \chi^{\alpha Z} \Gamma_{\alpha \beta a b d} \nabla_{e} \chi^{\beta T} \\
& \left.+\frac{1}{2} F_{a b}{ }^{Y} F_{c d}{ }^{T} F_{e f}{ }^{V} \chi^{\alpha Z} \Gamma_{\alpha \beta a b c d e} \nabla_{f} \chi^{\beta W}+\frac{1}{2} F_{a b}{ }^{Y} F_{c d}^{T} f_{e f}^{Z} \chi^{\alpha V} \Gamma_{\alpha \beta a b c d e} \nabla_{f} \chi^{\beta W}\right] .
\end{aligned}
$$

In these formulas capital Roman letters are Lie algebra indices, $f^{X Y Z}$ are structure constants of the gauge group Lie algebra.

The way to get the formula (55) will be described below.

One can construct a SUSY-invariant deformation of SYM theory reduced to a point by the formula:

$$
\delta \mathcal{L}=A \operatorname{tr} G
$$

where the operator $A$ is given by

$$
A=\theta_{1} \ldots \theta_{16}
$$

Here $\operatorname{tr} G$ is a gauge invariant expression (we can consider $G$ as an element of $U(Y M)$ ). If $G$ is $\operatorname{Spin}(10)$-invariant the deformation $\delta \mathcal{L}=A \operatorname{tr} G$ is super Poincaré invariant.

Notice that supersymmetry transformations $\theta_{\alpha}$ commute with $\operatorname{tr}$, hence $A \operatorname{tr} G=\operatorname{tr} A G$. 
If $G$ is a gauge invariant local expression (an element of $U(T Y M)$ ) the formula (56) specifies a supersymmetric deformation of non-reduced SYM theory.

Let us prove these statements. It is sufficient to check that in non-reduced case $\theta_{\alpha} \delta \mathcal{L}$ is a total derivative on shell, i.e. $\theta_{\alpha} \delta \mathcal{L}=\frac{\partial}{\partial x^{2}} H^{i}$ on the equations of motion of $\mathcal{L}_{S Y M}$. It will follow that in the reduced case $\theta_{\alpha} \delta \mathcal{L}$ vanishes on shell.

To prove this fact we notice that the anti-commutator $\left[\theta_{\alpha}, \theta_{\beta}\right]$ is a total covariant derivative as follows from (7). It follows from the same formula that $\theta_{\alpha}^{2}$ is a total covariant derivative. Calculating $\theta_{\alpha} A \operatorname{tr} G$ we are moving $\theta_{\alpha}$ using (77) until we reach $\theta$ with the same index. Then we use a formula for $\theta_{\alpha}^{2}$ :

$$
\begin{aligned}
& \theta_{\alpha} \delta \mathcal{L}=\operatorname{tr}\left(\theta_{\alpha} \theta_{1} \cdots \theta_{16} G\right)= \\
& =\sum_{\gamma=1}^{\alpha-1}(-1)^{\gamma} \Gamma_{\alpha \gamma}^{k} \operatorname{tr}\left(\theta_{1} \cdots \theta_{\gamma-1} D_{k} \theta_{\gamma+1} \cdots \theta_{16} G\right)+ \\
& +\frac{1}{2} \sum_{\gamma=1}^{\alpha-1}(-1)^{\alpha} \Gamma_{\alpha \alpha}^{k} \operatorname{tr}\left(\theta_{1} \cdots \theta_{\alpha-1} D_{k} \theta_{\alpha+1} \cdots \theta_{16} G\right) .
\end{aligned}
$$

Expressions $\operatorname{tr}\left(D_{k} \theta_{\alpha} \cdots \theta_{16} G\right)=\frac{\partial}{\partial x_{k}} \operatorname{tr}\left(\theta_{\alpha} \cdots \theta_{16} G\right)$ are total derivatives. Expressions $\operatorname{tr}\left(\theta_{1} \cdots \theta_{\gamma-1} D_{k} \theta_{\gamma+1} \cdots \theta_{16} G\right)$ are multiple supersymmetry transformations of total derivatives. Hence due to equation (8) $A \operatorname{tr} G$ is also a total derivative on the equations of motion for $\mathcal{L}_{S Y M}$.

The reader will recognize in (57) a 10-dimensional analog of $\theta$-integration in theories admitting superspace formulation with manifest supersymmetries.

The above considerations can be used to describe all infinitesimal deformations of YM theory reduced to a point. Namely we have the following theorem.

Theorem 9 Every infinitesimal super Poincaré-invariant deformation of $\mathcal{L}_{S Y M}$ reduced to a point is a linear combination of $\mathcal{L}_{16}$ and a deformation having a form $\operatorname{Atr}(G)$, where $G$ is an arbitrary $\operatorname{Spin}(10)$-invariant combination of products of $A_{i}$ and $\chi^{\alpha}$.

To formulate the corresponding statement in the case of unreduced $\mathcal{L}_{S Y} M$ we should generalize the above consideration a little bit. We notice that infinitesimal deformation of $\mathcal{L}_{S Y M}$ descends to a deformation of reduced $\mathcal{L}_{S Y M}$ 
(we formally replace $\nabla_{i}$ by constant matrix $A_{i}$ and matrix function $\chi$ by a constant matrix). It is not true that all Lagrangians in the reduced theory can be lifted a Lagrangian in ten-dimensional theory (in other words not every Lagrangian of reduced theory descends from a Lagrangian of non-reduced theory). For example an expression $\operatorname{tr} A_{i} A_{i}$ defines a Lagrangian of reduced theory, but

$$
\Delta=\operatorname{tr} \nabla_{i} \nabla_{i}
$$

does not make sense as a ten-dimensional Lagrangian.

Of course, if $G$ itself is a gauge-covariant local expression, the expression $A G$ is also local and, as we noticed already, specifies a supersymmetric deformation. However there are situations when $G$ is not of this kind but still $A G$ after adding commutator terms, total derivatives and performing field redefinition becomes a gauge-invariant local expression; then this expression can be considered as a Lagrangian of a deformation of ten-dimensional SYM Lagrangian $\mathcal{L}_{S Y M}$. We are saying that in this case the supersymmetric deformation of reduced theory can be lifted to a deformation of non-reduced theory.

Our homological computations [36] show that the number of linearly independent Poincaré invariant deformations of reduced SYM theory that do not have the form $A \operatorname{tr}(G)$ where $G \in U(T Y M)$, but can be lifted to ten-dimensional theory is equal to two.

To construct the first one we take $G$ to be the "Laplacian" (59).

We have

$$
A \operatorname{tr}(\Delta)=2 \operatorname{tr}\left(\left(A \nabla_{i}\right) \nabla_{i}\right)+\cdots
$$

where the dots represent gauge-invariant local terms. This follows from formula (5). It remains to prove we that $\left.\left(A \nabla_{i}\right) \nabla_{i}\right)$ is equivalent to a gauge-covariant local expression (recall that we allow field redefinition and adding commutator terms). It follows from the remarks at the beginning of the section that instead of working with $\left(A \nabla_{i}\right) \nabla_{i}$ we can work with $\left(A \boldsymbol{D}_{i}\right) \boldsymbol{D}_{i}$ considered as an element of $U(Y M) /[U(Y M), U(Y M)]$. Moreover, it is convenient to work in the algebra $U(L)$ generated by $\boldsymbol{\theta}_{\alpha}$ obeying $\Gamma_{i_{1}, \ldots, i_{5}}^{\alpha \beta}\left[\boldsymbol{\theta}_{\alpha}, \boldsymbol{\theta}_{\beta}\right]=0$ (see Section 2). 
The commutators with $\boldsymbol{\theta}_{\alpha}$ act on $U(Y M)$ as supersymmetries $\theta_{\alpha}$.

In the algebra $U(L)$ we can represent $A\left(\boldsymbol{D}_{i}\right)$ as the multiple commutator:

$$
A\left(\boldsymbol{D}_{i}\right)=\left[\boldsymbol{\theta}_{1}, \ldots,\left[\boldsymbol{\theta}_{16}, \boldsymbol{D}_{i}\right] \ldots\right] .
$$

We have more then four $\boldsymbol{\theta}$ 's in a row applied to $\boldsymbol{\theta}_{\beta}$. We see that $A\left(\boldsymbol{D}_{i}\right)$ is a commutator

$$
A\left(\boldsymbol{D}_{i}\right)=\left[\boldsymbol{D}_{k}, \psi_{k i}\right]+\left[\chi^{\alpha}, \psi_{\alpha i}\right]
$$

in $U(Y M)$, where $\psi_{k i}, \psi_{\alpha i}$ are gauge-covariant local expressions. We have the following line of identities where we can neglect commutator terms:

$A\left(\boldsymbol{D}_{i}\right) \boldsymbol{D}_{i}=\left[\boldsymbol{D}_{k}, \psi_{k i}\right] \boldsymbol{D}_{i}+\left[\chi^{\alpha}, \psi_{\alpha i}\right] \boldsymbol{D}_{i}=-\psi_{k i}\left[\boldsymbol{D}_{k} \boldsymbol{D}_{i}\right]+\left[\boldsymbol{D}_{k}, \psi_{k i} \boldsymbol{D}_{i}\right]-\psi_{\alpha i}\left[\boldsymbol{D}_{i}, \boldsymbol{\chi}^{\alpha}\right]+\left[\chi^{\alpha}, \psi_{\alpha i} D_{i}\right]$.

We obtain that $\operatorname{tr}\left(A\left(\boldsymbol{D}_{i}\right) \boldsymbol{D}_{i}\right)=\operatorname{tr}\left(\psi_{k i}\left[\boldsymbol{D}_{k} \boldsymbol{D}_{i}\right]\right)-\operatorname{tr}\left(\psi_{\alpha i}\left[\boldsymbol{D}_{i}, \chi^{\alpha}\right]\right)$.

One can check that supersymmetric deformation obtained from $\Delta=G_{1}$ is equivalent to (55).

One can prove that similar considerations can be applied to

$G_{2}=a \operatorname{tr}\left(F_{i_{2} i_{3}} F_{i_{2} i_{3}} D_{i_{1}} D_{i_{1}}\right)+b \operatorname{tr}\left(\Gamma_{\alpha \beta}^{i_{2}}\left[D_{i_{1}}, \chi^{\alpha}\right] \chi^{\beta} D_{i_{2}} D_{i_{1}}\right)+c \operatorname{tr}\left(\Gamma_{\alpha \beta}^{i_{1} i_{2} i_{3}} F_{i_{2} i_{3}} \chi^{\alpha} \chi^{\beta} D_{i_{1}}\right)$

for an appropriate choice of constants $a, b, c$. Corresponding deformation will be denoted by $\delta \mathcal{L}_{28}$.

In the Appendix $\mathrm{F}$ we describe a general way to obtain formulas for supersymmetric deformations. In particular, we give another expression for $G_{2}$ that does not contain indeterminate constants.

Theorem 10 Every deformation of Lagrangian $\mathcal{L}_{S Y M}$ that can be obtained by means of lifting of super Poincaré invariant deformation of reduced SYM theory is a linear combination of $\delta \mathcal{L}_{16}$ given by the formula (53) $, \delta \mathcal{L}_{20}=\operatorname{tr} H_{1}, \delta \mathcal{L}_{28}=$ $\operatorname{tr} H_{2}$ and a deformation of a form $\operatorname{tr}(A G)$ where $G$ is an arbitrary Poincaréinvariant combination of products of covariant derivatives of curvature $F_{i j}$ and spinors $\chi^{\alpha}$. Here $H_{1}$ can be obtained from $A G_{1}$ and $H_{2}$ can be obtained from $A G_{2}$ by adding commutator terms, total derivatives and terms coming from field redefinition. ( $G_{1}$ is defined by the formula (59) and $G_{2}$ by the formula (62)) 
There is a finer decomposition of the linear space of equivalence classes of Lagrangians. Any Lagrangian $\mathcal{L}$ under consideration has the form $\mathcal{L}=\operatorname{tr} Y(\nabla, \chi)$, where $Y$ is some non-commutative polynomial in $\nabla_{i}$ and $\chi^{\alpha}$. Let the noncommutative polynomial $Y$ be a linear combination of commutators. Then of course $\operatorname{tr} Y \equiv 0$, however if $Y, Y^{\prime}$ are commutators then

$$
\operatorname{tr} Y Y^{\prime}
$$

could be nonzero. The grading $\operatorname{deg}_{[]}$of a Lagrangian of the form $\operatorname{tr} Y Y^{\prime}$ by definition is equal to two (to the number of commutators in the product under the trace in (63) 13 For example the basic Lagrangian $\mathcal{L}_{S Y M}$ has degree $\left.\operatorname{deg}_{[}\right]$ equal to two. Likewise we can define Lagrangians of arbitrary degree $\operatorname{deg}_{[]}$. The equations of motion of YM theory are compatible with classification of Lagrangians by $\operatorname{deg}_{[]}$in the sense that Lagrangians of different degree are not equivalent.

The following table is a result of classification of linearly independent onshell supersymmetric Lagrangians of low degree. The numbers in the body of the table represent dimensions of spaces of super Poincaré invariant Lagrangians of degrees $\left(\operatorname{deg}_{[]}, \operatorname{deg}_{\alpha^{\prime}}\right)$.

\begin{tabular}{|c|c|c|c|c|c|c|c|c|c} 
& 1 & 2 & 3 & 4 & 5 & 6 & 7 & 8 & $k=\operatorname{deg}_{\alpha^{\prime}}$ \\
\hline 2 & & & 1 & & 1 & 3 & 18 & 172 & $\ldots$ \\
\hline 3 & & & & & & & 13 & 281 & $\ldots$ \\
\hline 4 & & 1 & & & 1 & 2 & 20 & 267 & $\ldots$ \\
\hline 5 & & & & & & & 1 & 68 & $\ldots$ \\
\hline 6 & & & & & & & 1 & 17 & $\ldots$ \\
\hline 7 & & & & & & & & & $\ldots$ \\
\hline$p=\operatorname{deg}_{[]}$ & $\ldots$ & $\ldots$ & $\ldots$ & $\ldots$ & $\ldots$ & $\ldots$ & $\ldots$ & $\ldots$ & $\ldots$
\end{tabular}

The entry in the second column corresponds to the Lagrangian (53), the entry in the third column corresponds to the Lagrangian (55).

\footnotetext{
${ }^{13}$ Lagrangians of this kind make sense not only for the gauge group $\mathrm{U}(N)$, but also for an arbitrary compact gauge group $G$ because they can be written intrinsically in terms of the commutator and the invariant inner product of the Lie algebra of $G$.
} 


\section{The Homological Approach to Infinitesimal Deformations}

In this section we shall describe a reduction of the problem of infinitesimal SUSY deformations of SYM to a homological problem. A general way to give homological formulation of a problem of classification of deformations will be described in Section 6 and in Appendix $\mathrm{A}$ the relation of this way to the approach of present section will be studied in Appendix C.

First of all we consider infinitesimal deformations of SYM reduced to a point. As we have seen, this theory can be expressed in terms of algebra $U(Y M)$. We shall regard the deformations of this theory as deformations of algebra $U(Y M)$. In other words we think about deformation as of family of multiplications on linear space $U(Y M)$ depending smoothly on parameter $\epsilon$. In the case of infinitesimal deformations we assume that $\epsilon^{2}=0$ (i.e. we neglect higher order terms with respect to $\epsilon$ ). We say that deformation is supersymmetric if it is possible to deform the SUSY algebra action on $U(Y M)$ in such a way that it consists of derivations of the deformed multiplication.

Theorem 11 Every cohomology class $\lambda \in \mathrm{H}^{2}(L, U(Y M))=\mathrm{H}^{2}(L, \operatorname{Sym}(Y M))$ specifies an infinitesimal supersymmetric deformation of $U(Y M)$.

We consider here $U(Y M)$ as a representation of Lie algebra L. Due to Poincaré-Birkhoff-Witt theorem this representation is isomorphic to $\operatorname{Sym}(Y M)$.

We shall start with general statement about deformations of associative algebra $A$. The multiplication in this algebra can be considered as a bilinear map $m: A \otimes A \rightarrow A$. An infinitesimal deformation $m+\delta m$ of this map specifies an associative multiplication if

$$
\delta m(a, b) c+\delta m(a b, c)=a \delta m(b, c)+\delta m(a, b c) .
$$

This condition means that $\delta m$ is a two-dimensional Hochschild cocycle with coefficients in $A$ (see Appendix A). Identifying equivalent deformations we obtain that infinitesimal deformations of associative algebra are labeled by the elements 
of Hochschild cohomology $\mathrm{HH}^{2}(A, A)$. ( Two deformations are equivalent if they are related by linear transformation of $A$.)

Applying this statement to the algebra $U(Y M)$ we obtain that the infinitesimal deformations of this algebra are labeled by the elements of $\mathrm{HH}^{2}(U(Y M), U(Y M))$.

Let us consider now the Hochschild cohomology $\mathrm{HH}^{2}(U(L), U(Y M)$ ). (Notice that $U(Y M)$ is an ideal in $U(L)$, hence it can be regarded as a $U(L)$ bimodule.) We can consider the natural restriction map $\mathrm{HH}^{2}(U(L), U(Y M)) \rightarrow$ $\mathrm{HH}^{2}(U(Y M), U(Y M))$; we shall check that the image of this map consists of supersymmetric deformations. Let us notice first of all that $L=L^{1}+Y M$ and the derivations $\gamma_{a}$ corresponding to the elements $a \in L^{1}$ act on $Y M$ as supersymmetries; this action can be extended to $U(Y M)$ and specifies an action on Hochschild cohomology, in particular, on the space of deformations $\mathrm{HH}^{2}(U(Y M), U(Y M))$. (The derivation $\gamma_{a}$ is defined by the formula $\gamma_{a}(x)=$ $[a, x]$.) On $L$ one can consider $\gamma_{a}$ as an inner derivation, hence its action on the cohomology $\mathrm{HH}^{2}(U(L), U(Y M))$ is trivial. (This follows from well known results, see, for example, 31.) This means that supersymmetry transformations act trivially on the image of $\mathrm{HH}^{2}(U(L), U(Y M))$ in $\mathrm{HH}^{2}(U(Y M), U(Y M)$ ) (in the space of deformations).

To obtain the statement of the theorem it is sufficient to notice that the Hochschild cohomology of the enveloping algebra of Lie algebra can be expressed in terms of Lie algebra cohomology (see (131) ).

Theorem 11 gives a homological description of supersymmetric deformations of the equations of motion. We can use homological methods to answer the question: when the deformed EM come from a Lagrangian. As we have seen in Section 3 the space of infinitesimal Lagrangian deformations of SYM theory reduced to a point can identified with $U(Y M) /[U(Y M), U(Y M)]=$ $\mathrm{HH}_{0}(U(Y M), U(Y M))$. Lagrangian deformation generates a deformation of EM, hence there exists a map $U(Y M) /[U(Y M), U(Y M)] \rightarrow \mathrm{HH}^{2}(U(Y M), U(Y M))=$ $\mathrm{H}^{2}(Y M, U(Y M))$. It turns out (see [33] and [36] ) that the image of this map has a finite codimension in $\mathrm{H}^{2}(Y M, U(Y M))$ and it is onto for $\operatorname{Spin}(10)$-invariant elements. This means that all Poincaré invariant infinitesimal deformations of 
EM are Lagrangian deformations.

Let us consider now deformations of supersymmetric deformations of supersymmetric YM theory in ten-dimensional case (SYM theory). The description of these deformations is similar to reduced case.

Theorem 12 Every element $\lambda \in \mathrm{H}^{2}(L, U(T Y M))$ specifies a supersymmetric deformation of SUSY YM.

The group Spin(10) acts on cohomology; Poincaré invariant deformations are identified with Spin(10)-invariant cohomology classes.

In the proof we interpret the deformations of SYM theory as deformations of the algebra $U(T Y M)$ and identify infinitesimal deformations with elements of Hochschild homology $\mathrm{HH}_{0}(U(T Y M), U(T Y M))$. However, the proof is more complicated; it is based on results of Section 6 and Appendix C It is shown in Appendix $\mathrm{C}$ that the elements of higher cohomology groups also correspond to supersymmetric deformations, however only elements of $\mathrm{H}^{2}$ give non-trivial super-Poincaré invariant infinitesimal deformations of equations of motion.

The next section is devoted to the calculation of cohomology entering the formulation of the theorems 11 and 12. In present section we will describe the solution of simpler problem of calculation of corresponding Euler characteristics. The Euler characteristics are especially interesting, because the contribution of infinitesimal deformations given by the general formula (57) with $G \in Y M$ and $G \in T Y M$ cancels in Euler characteristic.

To get more information about homology we calculate Euler characteristics of homology groups considered as graded Spin(10)- modules (as representations of Spin(10)). Recall that $L$ is graded; this gives a grading on homology. However, there is also another grading coming from Poincaré-Birkhoff-Witt identification of $\mathfrak{g}$-modules $U(\mathfrak{g})=\sum \operatorname{Sym}^{k} \mathfrak{g}$. As explained in the Section 2.4 the Poincaré series of graded $\operatorname{Spin}(10)$-modules $U(Y M), U(T Y M), U(L)$ and Euler characteristics $\mathrm{H}^{\bullet}(L, U(Y M)), \mathrm{H}^{\bullet}(L, U(T Y M))$ can be considered as functions of $g \in \operatorname{Spin}(10)$ and series with respect $t$ (we disregard the the second grading for a moment). It follows from (48) that Euler characteristics can be expressed 
in terms of Poincare series:

$$
\begin{aligned}
\chi_{\mathrm{H} \bullet(L, U(Y M))}(g, t) & =\frac{U(Y M)(g, t)}{U(L)\left(g^{-1}, t^{-1}\right)} \\
\chi_{\mathrm{H}_{\bullet}(L, U(Y M))}(g, t) & =\frac{U(Y M)(g, t)}{U(L)(g, t)}
\end{aligned}
$$

The factor-algebra $L / T Y M$ can be identified with the super Lie algebra of supersymmetries (16 odd generators transforming according spinor representation $S, 10$ even generators transforming according vector representation $V$ ). Similarly, the factor algebra $L / Y M$ has 16 odd generators with trivial anticommutation relations; they transform as spinors.

This remark (together with the formula for $U(L)(g, t)$ given in Corollary 8) permits us to prove the following formulas

$$
\begin{aligned}
& U(Y M)(g, t)=U(L)(g, t) \operatorname{Sym} S^{*}(g,-t)= \\
& =\frac{1}{1-V\left(g^{-1}\right) t^{2}-S\left(g^{-1}\right) t^{3}+S(g) t^{5}+V(g) t^{6}-t^{8}} \\
& U(T Y M)(g, t)=U(L)(g, t) \operatorname{Sym} S^{*}(g,-t) \Lambda V(g,-t)= \\
& =\frac{\Lambda V\left(g,-t^{2}\right)}{1-V\left(g^{-1}\right) t^{2}-S\left(g^{-1}\right) t^{3}+S(g) t^{5}+V(g) t^{6}-t^{8}}
\end{aligned}
$$

We can use these formulas together with (65) to prove that

$$
\begin{gathered}
\chi_{\mathrm{H} \bullet(L, U(Y M))}(g, t)=-\frac{1}{t^{8}} \operatorname{Sym} S\left(g,-t^{-1}\right) \\
\chi_{\mathrm{H} \bullet(L, U(Y M))}(g, t)=\operatorname{Sym}^{*}(g,-t) \\
\chi_{\mathrm{H} \bullet(L, U(T Y M))}(g, t)=-\frac{1}{t^{8}} \operatorname{Sym} S\left(g,-t^{-1}\right) \Lambda V\left(g,-t^{-2}\right) \\
\chi_{\mathrm{H} \bullet(L, U(T Y M))}(g, t)=\operatorname{Sym}^{*}(g,-t) \Lambda V\left(g,-t^{2}\right)
\end{gathered}
$$

To take into account the second grading we should use formulas

$$
\begin{aligned}
\sum \chi_{\mathrm{H}_{(}\left(L, \mathrm{Sym}^{j} T Y M\right)}(g, t) z^{j} & =\frac{U(T Y M)(g, t, z)}{U(L)\left(g^{-1}, t^{-1}\right)} \\
\sum \chi_{\mathrm{H}_{\bullet}\left(L, \mathrm{Sym}^{j} T Y M\right)}(g, t) z^{j} & =\frac{U(T Y M)(g, t, z)}{U(L)(g, t)} \\
\sum \chi_{\mathrm{H}^{\bullet}\left(L, \mathrm{Sym}^{j} Y M\right)}(g, t) z^{j} & =\frac{U(Y M)(g, t, z)}{U(L)\left(g^{-1}, t^{-1}\right)} \\
\sum \chi_{\mathrm{H}_{\bullet}\left(L, \mathrm{Sym}^{j} Y M\right)}(g, t) z^{j} & =\frac{U(Y M)(g, t, z)}{U(L)(g, t)}
\end{aligned}
$$


that also follow from (48) (we have replaced the numerator in (65) by the Poincaré series with respect to the double grading).

To calculate $U(Y M)(g, t, z)$ and $U(T Y M)(g, t, z)$ we notice that in general knowing the Poincaré series of Lie algebra $\mathfrak{g}$ we can calculate the Poincaré series of $U(\mathfrak{g})$ that takes into account the additional grading coming from the Poincaré-Birkhoff-Witt isomorphism $U(\mathfrak{g}) \cong \operatorname{Sym}[\mathfrak{g}]$. 14 From the other side the knowledge of the Poincaré series of $U(\mathfrak{g})$ defined without additional grading is sufficient to find the Poincaré series $\mathfrak{g}(t)$ of $\mathfrak{g}$.

If we neglect the action of a group we obtain

$$
\mathfrak{g}(t)=\sum_{n \geq 1} \frac{\mu(n)}{n} \ln U(\mathfrak{g})\left(-(-t)^{n}\right)
$$

where $\mu$ stands for the Möbius function.

We derive from it that

$$
U(\mathfrak{g})(t, z)=\exp \left(\sum_{n, l \geq 1} \frac{\mu(l)}{n l} z^{n} \ln U(\mathfrak{g})\left(-(-t)^{n l}\right)\right)
$$

There is a more general formula that incorporates the group action

$$
U(\mathfrak{g})(g, t, z)=\exp \left(\sum_{n, l \geq 1} \frac{\mu(l)}{n l} z^{n} \ln U(\mathfrak{g})\left(g^{n l},-(-t)^{n l}\right)\right)
$$

It can be obtained from the formula (172) in Appendix [

\section{Calculation of the Cohomology}

The cohomology governing infinitesimal deformations of ten-dimensional SYM and its reductions to a point were calculated in [36. In present section we use the approach of [36] to justify the statements of Theorems 9 and 10. The calculation will be based on Corollaries 516 (Section 2.2). We mentioned in Section 2.1 that the algebra $\mathcal{S}$ is related to the manifold of pure spinors $\mathcal{C}$ and to the corresponding compact manifold $\mathcal{Q}$. Namely $\mathcal{S}_{k}$ can be interpreted as a

\footnotetext{
${ }^{14}$ In the notations of Appendix [ we should calculate the character of $\sigma_{t}(\mathfrak{g})$.
} 
space of holomorphic sections of line bundle $\mathcal{O}(k)$ over $\mathcal{Q}$. In other words

$$
\mathcal{S}_{k}=\mathrm{H}^{0}(\mathcal{Q}, \mathcal{O}(k)) \text { for } k \geq 0
$$

One can prove that all other cohomology groups $\mathrm{H}^{i}(\mathcal{Q}, \mathcal{O}(k))$ of $\mathcal{Q}$ with coefficients in line bundles $\mathcal{O}(k)$ are zero except

$$
\mathrm{H}^{10}(\mathcal{Q}, \mathcal{O}(k))=\mathcal{S}_{-k-8}^{*} \text { for } k \leq-8 .
$$

The proof is based on Borel-Weil-Bott theorem 15

The relation between $\mathcal{S}$ and $\mathcal{Q}$ can be used to express cohomology of a graded $L$-module $N=\bigoplus_{m \geq m_{0}} N_{m}$ in terms of cohomology groups related to $\mathcal{Q}$. Recall that Corollary 5 permits us to reduce the calculation of the cohomology at hand to the calculation of the cohomology of the complex (of differential module)

$$
N_{c \boldsymbol{m}}^{\bullet}=\left(N_{m} \otimes \mathcal{S}_{0} \rightarrow N_{m+1} \otimes \mathcal{S}_{1} \rightarrow \ldots\right)
$$

We can construct a differential vector bundle (a complex of holomorphic vector bundles $\mathcal{N}^{\bullet}$ ) over $\mathcal{Q}$ in such a way that one obtains the above complex

\footnotetext{
15 Borel-Weil-Bott theory deals with calculation of the cohomology of $G / P$ with coefficients in $G$-invariant holomorphic vector bundles over $G / P$. Here $G / P$ is a compact homogeneous space, $P$ is a complex subgroup of complex Lie group $G$. These bundles correspond to complex representations of the subgroup $P$; more precisely, the total space of vector bundle $\mathcal{E}$ corresponding to $P$-module $E$ ( to a representation of $P$ in the space $E$ ) can be obtained from $E \times G$ by means of factorization with respect to the action of $P$.

Usually Borel-Weil -Bott theorem is applied in the case when the representation of $P$ is one-dimensional (in the case of line bundles); it describes the cohomology as a representation of the group $G$. However, more general case also can be treated [8].

We suppose that the group $G$ is connected and the homogeneous space $G / P$ is simply connected; then $G / P$ can be represented as $M / P \bigcap M$ where $M$ is a compact Lie group and $G$ is a complexification of $M$. If $E$ is a complex $P$-module then
}

$$
\mathrm{H}^{\bullet}(G / P, \mathcal{E})=\sum K \otimes \mathrm{H}^{\bullet}(\mathfrak{p}, \mathfrak{v}, \operatorname{Hom}(K, E))
$$

where $K$ ranges over irreducible $M$-modules. This formula gives an expression of cohomology with coefficients in vector bundle in terms of relative Lie algebra cohomology, $\mathfrak{p}$ stands for real Lie algebra of $P$ and $\mathfrak{v}$ stands for Lie algebra of $P \bigcap M$. 
of modules considering holomorphic sections of vector bundles:

$$
\begin{aligned}
& \mathcal{N}_{c}^{\bullet} \boldsymbol{l}=\left(\cdots \rightarrow N_{l-1} \otimes \mathcal{O}(-1) \rightarrow N_{l} \otimes \mathcal{O}(0) \rightarrow N_{l+1} \otimes \mathcal{O}(1) \rightarrow \ldots\right)= \\
& =\left(\cdots \rightarrow N_{l-1}(-1) \rightarrow N_{l}(0) \rightarrow N_{l+1}(1) \rightarrow \ldots\right) .
\end{aligned}
$$

We use here the notation $N(k)=N \otimes \mathcal{O}(k)$. Notice, that the construction of the complex of vector bundles depends on the choice of index $l$, but this dependence is very simple: $\mathcal{N}_{c}^{\bullet} \boldsymbol{l + 1}=\mathcal{N}_{c}^{\bullet} \boldsymbol{l}(-1)$. The differential $d_{e}$ is a multiplication by

$$
e=\lambda^{\alpha} \boldsymbol{\theta}_{\alpha}
$$

Let us assume that the modules $N_{i}$ are also Spin(10)-modules (more precisely, $N$ is a module with respect of semidirect product of $L$ and $\operatorname{Spin}(10)$ ). Then vector bundles in the complex (70) are Spin(10)-invariant; corresponding complex $N_{P}$ of $P$-modules has the form

$$
N_{P}=\left(\cdots \rightarrow N_{l-1} \otimes \mu_{-1} \rightarrow N_{l} \otimes \mu_{0} \rightarrow N_{l+1} \otimes \mu_{1} \rightarrow \ldots\right)
$$

(Recall that $\mathcal{Q}=\mathrm{SO}(10) / \mathrm{U}(5)$ can be obtained also by means of taking quotient of complex spinor group $\operatorname{Spin}(10, \mathbb{C})$ with respect to the subgroup $P$ defined as a stabilizer of a point $\lambda_{0} \in \mathcal{Q}$; see Section 2.3. The complex of $P$-modules comes from consideration of the complex of fibers over $\lambda_{0}$. )

Let us consider hypercohomology of $\mathcal{Q}$ with the coefficients in the complex $\mathcal{N}_{\boldsymbol{l}}^{\bullet}=\mathcal{N}_{c}^{\bullet} \boldsymbol{l}$. These hypercohomology can be expressed in terms of the Dolbeault cohomology of $\mathcal{N}_{\boldsymbol{l}}^{\bullet}$. Namely we should consider the bicomplex $\Omega^{\bullet}\left(\mathcal{N}_{\boldsymbol{l}}^{\bullet}\right)$ of smooth sections of the bundle of $(0, p)$ - forms with coefficients in $\mathcal{N}_{l}^{q}$. Two differentials are $\bar{\partial}$ and $d_{e}$. Hypercohomology $\mathbb{H}^{i}\left(\mathcal{Q}, \mathcal{N}_{l}\right)$ can be identified with cohomology of the total differential $\bar{\partial}+d_{e}$ in $\Omega^{\bullet}\left(\mathcal{N}_{\boldsymbol{l}}^{\bullet}\right)$.

As usual we can analyze cohomology of the total differential by means of two spectral sequences whose $E_{2}$ terms are equal to $\mathrm{H}^{i}\left(\mathrm{H}^{j}\left(\Omega\left(\mathcal{N}_{l}\right), \bar{\partial}\right), d_{e}\right)$ and $\mathrm{H}^{i}\left(\mathrm{H}^{j}\left(\Omega\left(\mathcal{N}_{l}\right), d_{e}\right), \bar{\partial}\right)$.

Proposition 13 There is a long exact sequence of cohomology

$$
\begin{aligned}
& \cdots \rightarrow \mathrm{H}^{i}\left(N_{c l}\right) \rightarrow \mathbb{H}^{i}\left(\mathcal{Q}, \mathcal{N}_{l}\right) \rightarrow \mathrm{H}^{i-10}\left(N_{h-8-l}\right) \stackrel{\delta}{\rightarrow} \\
& \stackrel{\delta}{\rightarrow} \mathrm{H}^{i+1}\left(N_{c} \iota\right) \rightarrow \ldots
\end{aligned}
$$


Proof. It follows readily from equalities (68, 69) that nontrivial rows in $E_{2}$ of the first spectral sequence are $\left(\mathrm{H}^{0}\left(\Omega\left(\mathcal{N}_{\boldsymbol{l}}^{\bullet}\right), \bar{\partial}\right)=N_{c} \boldsymbol{l}\right.$ and $\left(\mathrm{H}^{10}\left(\Omega\left(\mathcal{N}_{\boldsymbol{l}}^{\bullet}\right), \bar{\partial}\right)=\right.$ $N_{h-8-l}$. ( We use the notations of Corollaries 5 and 6.) The operator $\delta$ is the differential in $E_{2}$. To complete the proof we notice that this is the only non-vanishing differential in the spectral sequence.

We shall be interested in graded $L$ module $N=Y M$; the corresponding graded differential vector bundle (complex of vector bundles) is denoted by $\mathcal{Y} \mathcal{M}$. Notice, that this bundle is Spin(10)-invariant; it corresponds to the following representation of the group $P$ :

$$
L^{2}+L^{3} \otimes \mu_{1}+L^{4} \otimes \mu_{2}+\ldots
$$

( As we have noticed there is a freedom in the construction of complex of vector bundle; the above formula corresponds to $l=2$.)

Similarly starting with $L$ module $T Y M$ one can define graded differential vector bundle $\mathcal{T} \mathcal{Y M}$; it corresponds to the representation

$$
L^{3} \otimes \mu_{1}+L^{4} \otimes \mu_{2}+\ldots
$$

of the group $P$.

More generally, we can consider the module

$$
N=\bigoplus_{k \geq 0} N^{k}=\operatorname{Sym}^{j} Y M=\bigoplus_{k \geq 0} \operatorname{Sym}^{j} Y M^{k}
$$

equipped with adjoint action of $L$. Corresponding complexes of vector bundles are denoted $\mathrm{Sym}^{j} \mathcal{Y} \mathcal{M}$. Symmetric algebra Sym is understood in the graded sense.

Similarly, we can define complexes of vector bundles $\operatorname{Sym}^{j} \mathcal{T Y \mathcal { M }}$.

Let $\mathcal{W}^{*}$ be the vector bundle on $\mathcal{Q}$ induced from the representation $W^{*}$ of $P$. It follows from (43) that there is an embedding $W^{*} \subset L^{2}=Y M^{2} \subset Y M$. From this we conclude that there is an embedding $\mathcal{W}^{*} \rightarrow \mathcal{Y M}^{\bullet}$, where we consider $\mathcal{W}^{*}$ as a graded vector bundle with one graded component $W^{*}$ in grading 2 and zero differential (as one-term complex). 
Proposition 14 The embedding $\mathcal{W}^{*} \rightarrow \mathcal{Y} \mathcal{M}^{\bullet}$ is a quasi-isomorphism.

We relegate the proof to the Appendix E

Corollary 15 The embedding of $\operatorname{Sym}^{i}\left(\mathcal{W}^{*}\right)$ into $\operatorname{Sym}^{i}(\mathcal{Y} \mathcal{M})$ is a quasi-isomorphism.

Here $\operatorname{Sym}^{i}\left(\mathcal{W}^{*}\right)$ is considered as graded vector bundle with grading $2 i$. To deduce the corollary we use Künneth theorem.

We can reformulate Proposition 14 saying that the induced map of hypercohomology $\mathbb{H}^{\bullet}\left(\mathcal{W}^{*}\right) \rightarrow \mathbb{H} \bullet\left(\mathcal{Q}, \mathcal{Y} \mathcal{M}_{\mathbf{0}}^{\bullet}\right)$ is an isomorphism. Similarly, the map $\mathbb{H}^{\bullet}\left(\mathcal{Q}, \operatorname{Sym}^{i}\left(\mathcal{W}^{*}\right)(l)\right) \rightarrow \mathbb{H}^{\bullet}\left(\operatorname{Sym}^{i} \mathcal{Y} \mathcal{M}_{\boldsymbol{l}}\right)$ is an isomorphism.

Using this statement and (72) we obtain

Corollary 16 There is a long exact sequence of cohomology

$$
\begin{aligned}
& \quad \cdots \rightarrow \mathrm{H}^{i}\left(\operatorname{Sym}^{j} Y M_{c} \boldsymbol{l}\right) \rightarrow \mathrm{H}^{i+l-2 j}\left(\mathcal{Q}, \operatorname{Sym}^{j}\left(\mathcal{W}^{*}\right)(2 j-l)\right) \rightarrow \mathrm{H}^{i-10}\left(\operatorname{Sym}^{j} Y M_{h-8-l}\right) \stackrel{\delta}{\rightarrow} \\
& \stackrel{\delta}{\rightarrow} \mathrm{H}^{i+1}\left(\operatorname{Sym}^{j} Y M_{c} \boldsymbol{l}\right) \rightarrow \ldots
\end{aligned}
$$

Using Corollary [6 we can identify the cohomology $\mathrm{H}^{\bullet}\left(\operatorname{Sym}^{j} Y M_{h}\right)$ with homology $\mathrm{H}_{\bullet}(L, Y M)$. Likewise $\mathrm{H}^{\bullet}\left(\operatorname{Sym}^{j} Y M_{c}\right)$ is isomorphic to $\mathrm{H}^{\bullet}(L, \operatorname{Sym}(Y M))$. This means that we can formulate (75) as a long exact sequence

$$
\begin{aligned}
& \cdots \rightarrow \mathrm{H}^{i, \boldsymbol{l}}\left(L, \operatorname{Sym}^{j} Y M\right) \rightarrow \mathrm{H}^{i+\boldsymbol{l}-2 j}\left(\mathcal{Q}, \operatorname{Sym}^{j}\left(\mathcal{W}^{*}\right)(2 j-\boldsymbol{l})\right) \rightarrow \\
& \rightarrow \mathrm{H}_{2-i, \boldsymbol{l}-\mathbf{8}}\left(L, \operatorname{Sym}^{j} Y M\right) \stackrel{\delta}{\rightarrow} \mathrm{H}^{i+1, \boldsymbol{l}}\left(L, \operatorname{Sym}^{j} Y M\right) \rightarrow \ldots
\end{aligned}
$$

The hypercohomology $\mathbb{H}^{\bullet}\left(\mathcal{Q}, \operatorname{Sym}^{i}\left(\mathcal{W}^{*}\right)(l)\right)$ it is equal up to a shift in grading to the ordinary cohomology of the $\mathcal{Q}$ vector bundle $\operatorname{Sym}^{i}\left(\mathcal{W}^{*}\right)(l)$. Such cohomology can be computed via Borel-Weil-Bott theory.

\section{Proposition 17}

$$
\begin{aligned}
& \mathrm{H}^{0}\left(\mathcal{Q}, \operatorname{Sym}^{j}\left(\mathcal{W}^{*}\right)(l)\right)=[0,0,0, j, l-j], j, l-j \geq 0 \\
& \mathrm{H}^{4}\left(\mathcal{Q}, \operatorname{Sym}^{j}\left(\mathcal{W}^{*}\right)(l)\right)=[j-3,0,0, l+2,0], l \geq-2, j \geq 3 \\
& \mathrm{H}^{10}\left(\mathcal{Q}, \operatorname{Sym}^{j}\left(\mathcal{W}^{*}\right)(l)\right)=[j, 0,0,-8-l, 0], l \leq-8, j \geq 0
\end{aligned}
$$


Straightforward inspection of the cohomology groups shows that the following groups are generated by $\operatorname{Spin}(10)$ - invariant elements : $\langle e\rangle=\mathrm{H}^{0}\left(\mathcal{Q}, \operatorname{Sym}^{0}\left(\mathcal{W}^{*}\right)(0)\right)$, $\langle c\rangle=\mathrm{H}^{4}\left(\mathcal{Q}, \operatorname{Sym}^{3}\left(\mathcal{W}^{*}\right)(-2)\right),\left\langle e^{\prime}\right\rangle=\mathrm{H}^{10}\left(\mathcal{Q}, \operatorname{Sym}^{0}\left(\mathcal{W}^{*}\right)(-8)\right)$.

To analyze the super Poincaré invariant deformations we use Spin(10)-invariant part of exact sequence (76). It is easy to check that Spin(10)-invariant elements of hypercohomology listed above are mapped into zero in this long exact sequence. This means that this long exact sequence splits into short exact sequences

$$
\begin{aligned}
& \text { if } i=11, j=0, l=8 \text { then } \\
& 0 \rightarrow \mathrm{H}^{i+l-2 j-1}\left(\mathcal{Q}, \operatorname{Sym}^{j}\left(\mathcal{W}^{*}\right)(2 j-l)\right)^{\operatorname{Spin}(10)} \rightarrow \\
& \rightarrow \mathrm{H}_{3-i, l-8}\left(L, \operatorname{Sym}^{j} Y M\right)^{\operatorname{Spin}(10) \stackrel{\delta}{\rightarrow}} \mathrm{H}^{i, \boldsymbol{l}}\left(L, \operatorname{Sym}^{j} Y M\right)^{\operatorname{Spin}(10)} \rightarrow 0 \\
& \text { otherwise } \\
& 0 \rightarrow \mathrm{H}_{3-i, l-8}\left(L, \operatorname{Sym}^{j} Y M\right)^{\operatorname{Spin}(10)} \stackrel{\delta}{\rightarrow} \mathrm{H}^{i, \boldsymbol{l}}\left(L, \operatorname{Sym}^{j} Y M\right)^{\operatorname{Spin}(10)} \rightarrow \\
& \rightarrow \mathrm{H}^{i+l-2 j}\left(\mathcal{Q}, \operatorname{Sym}^{j}\left(\mathcal{W}^{*}\right)(2 j-l)\right)^{\operatorname{Spin}(10)} \rightarrow 0
\end{aligned}
$$

We see that that Spin(10)-invariant elements of hypercohomology $e, c, e^{\prime}$ contribute to (co)homology $\mathrm{H}^{0,0}(L, \mathbb{C}), \mathrm{H}^{2, \boldsymbol{8}}\left(L, \operatorname{Sym}^{3} Y M\right)^{\operatorname{Spin}(10)}$ and $\mathrm{H}_{0, \mathbf{0}}(L, \mathbb{C})$. The only non- trivial contribution corresponds to $c$ and gives the infinitesimal deformation $\delta \mathcal{L}_{16}$ (53).

\section{Proof of Theorem 9}

We shall give a proof of this theorem assuming that all infinitesimal supersymmetric deformations are given by Theorem 11, The key moment in the proof is the use of short exact sequence (77).

The operator $\delta$ in exact sequence (76) defines a map

$$
\delta: \mathrm{H}_{1}(L, \operatorname{Sym}(Y M)) \rightarrow \mathrm{H}^{2}(L, \operatorname{Sym}(Y M))
$$

whose kernel and cokernel are controlled by the exact sequence. We conclude that the space $\delta\left(\mathrm{H}_{1}(L, \operatorname{Sym}(Y M))^{\operatorname{Spin}(10)}\right)$ has codimension one in $\mathrm{H}^{2}(L, \operatorname{Sym}(Y M))^{\operatorname{Spin}(10)}$. We shall prove that the space of super Poincaré invariant deformations of equations of motion given by the formula (56) has the same codimension in 
$\mathrm{H}^{2}(L, \operatorname{Sym}(Y M))^{\operatorname{Spin}(10)}$ as $\delta\left(\mathrm{H}_{1}(L, \operatorname{Sym}(Y M))^{\operatorname{Spin}(10)}\right)$; this gives a proof of the theorem 9] (The formula (56) specifies a supersymmetric deformation of Lagrangian. However, a deformation of Lagrangian function produces a deformation of equations of motions; this manifests in a map

$$
\text { var }: \mathrm{H}_{0}(Y M, U(Y M)) \rightarrow \mathrm{H}^{2}(Y M, U(Y M)) .
$$

See Section 4 for more detail.)

Supersymmetry transformations $\boldsymbol{\theta}_{\alpha}$ act by derivations on Lie algebra $Y M$. From this we conclude $\boldsymbol{\theta}_{\alpha}$ induce operators that act on objects constructed naturally (functorially) from $Y M$. In particular they act on

$$
\mathrm{H}^{i}(Y M, U(Y M)) \stackrel{P}{\cong} \mathrm{H}_{3-i}(Y M, U(Y M))
$$

Here $P$ denotes the Poincaré isomorphism (see Appendix A). The composition $\boldsymbol{\theta}_{1} \cdots \boldsymbol{\theta}_{16}$ defines an operator in homology. We shall use the notation $A_{k}$ for this operator acting on $k$-dimensional homology:

$$
A_{k}: \mathrm{H}_{k}(Y M, U(Y M)) \stackrel{A}{\rightarrow} \mathrm{H}_{k}(Y M, U(Y M))
$$

In Section 3 we have interpreted the linear space $\mathrm{H}_{0}(Y M, U(Y M)) \cong \mathrm{H}_{0}(Y M$, $\operatorname{Sym}(Y M))$ as a linear space of infinitesimal deformations of action functions in the reduced theory. Obviously the operator $A_{0}$ coincides with $A$ defined in (56).

The maps $A_{k}$ have an alternative description. Let $N$ be an $L$-module. It is also an $Y M$-module. Since homology is a covariant functor with respect to the Lie algebra argument there is a map $\left(i_{*}\right)_{k}: \mathrm{H}_{k}(Y M, N) \rightarrow \mathrm{H}_{k}(L, N)$. Likewise there is a map in opposite direction on cohomology $\left(i^{*}\right)_{k}: \mathrm{H}^{k}(L, N) \rightarrow$ $\mathrm{H}^{k}(Y M, N)$. These observations enable us to define composition maps

$T_{k}: \mathrm{H}_{k}(Y M, U(Y M)) \stackrel{\left(i_{*}\right)_{k}}{\rightarrow} \mathrm{H}_{k}(L, U(Y M)) \stackrel{\delta}{\rightarrow} \mathrm{H}^{3-k}(L, U(Y M)) \stackrel{\left(i^{*}\right)_{3-k}}{\rightarrow} \mathrm{H}^{3-k}(Y M, U(Y M)) \stackrel{P}{\rightarrow} \mathrm{H}_{k}(Y M, U(Y M$ Notice, that the map $i_{2}^{*}$ acts from $\mathrm{H}^{2}(L, U(Y M))$ into $\mathrm{H}^{2}(Y M, U(Y M))$; we have shown in Section 4 that the elements in the image of this map correspond to supersymmetric deformations. The same arguments can be applied to the map $i_{k}^{*}$; they lead to the conclusion that

$$
T_{k}: \mathrm{H}_{k}(Y M, U(Y M)) \rightarrow \mathrm{H}_{k}(Y M, U(Y M))^{\text {sus } \mathfrak{y}}
$$


(in other words, the image of $T_{k}$ consists of supersymmetric elements). The map $A_{k}$ obviously has the same feature, therefore it is natural to conjecture that the maps $A_{k}$ and $T_{k}$ coincide. To prove this conjecture we notice that the operators $A_{k}$ and $T_{k}$ can be defined for arbitrary $L$-module $N$ as operators

$$
\mathrm{H}_{k}(Y M, N) \rightarrow \mathrm{H}_{k}(Y M, N)^{\mathfrak{s u s y}}
$$

- Using free resolutions one can reduce the proof to the consideration of the module $N \cong U(L)$ where $L$ acts on $U(L)$ by left multiplication (see [36] for details).

In general it is not easy to describe maps $i_{*}$ and $i^{*}$. It is easier to analyze their restrictions to Spin(10)-invariant elements. Let us consider maps $i_{* 1}$ : $\mathrm{H}_{1}(Y M, U(Y M))^{\mathrm{Spin}(10)} \rightarrow \mathrm{H}_{1}(L, U(Y M))^{\mathrm{Spin}(10)}$ and $i_{2}^{*}: \mathrm{H}^{2}(L, U(Y M))^{\mathfrak{s o}(10)} \rightarrow$ $\mathrm{H}^{2}(Y M, U(Y M))^{\mathfrak{s o}(10) \ltimes \mathfrak{s u s y}} \subset \mathrm{H}^{2}(Y M, U(Y M))^{\operatorname{Spin}(10)}$. One can prove the following

Lemma 18 The maps $i_{* 1}, i_{2}^{*}$ are surjective.

If we take this Lemma for granted we conclude that $A_{1}: \mathrm{H}_{1}(Y M, U(Y M))^{\mathfrak{s o}(10)} \rightarrow$ $\mathrm{H}_{1}(Y M, U(Y M))^{\mathfrak{s o}(10) \ltimes \mathfrak{s u s y}}$ has one-dimensional co-kernel (of the same dimension as the co-kernel of the map $\delta$ ).

The rather technical proof of the lemma (see [36]) is based on analysis of Serre-Hochschild spectral sequences associated with extension $Y M \subset L$ :

$$
\begin{aligned}
& \mathrm{H}_{i}(Y M, \operatorname{Sym} Y M) \otimes \operatorname{Sym}^{j} S^{*} \Rightarrow \mathrm{H}_{i+j}(L, \operatorname{Sym} Y M) \\
& \mathrm{H}^{i}(Y M, \operatorname{Sym} Y M) \otimes \operatorname{Sym}^{j} S \Rightarrow \mathrm{H}^{i+j}(L, \operatorname{Sym} Y M) .
\end{aligned}
$$

Notice that the surjectivity of $i_{2}^{*}$ has clear physical meaning: it can be interpreted as a statement that all super Poincaré invariant deformations in the sense of Section 4 are described by Theorem 10.

The above considerations gave us the information about the codimension of the image of the operator $A_{1}$. To prove Theorem 9 we need information about the codimension of the image of $A_{0}$. This information can be obtained from the results about operator $A_{1}$ by means of Connes differential

$$
B: \mathrm{H}_{k}(Y M, U(Y M)) \rightarrow \mathrm{H}_{k+1}(Y M, U(Y M))
$$


(see Appendix (A). Using the fact that supersymmetries commute with the Connes differential we obtain that

$$
A_{k+1} B=B A_{k}
$$

, in particular, $A_{1} B=B A_{0}$.

We need the following

Lemma 19 The map $B$ defines a surjective map $\mathrm{H}_{0}(Y M, U(Y M))^{\mathfrak{s o}(10)} \rightarrow$ $\mathrm{H}_{1}(Y M, U(Y M))^{\mathfrak{s o}(10)}$ with one-dimensional kernel generated by constants.

Proof. The proof (see [33] and [36]) is based on a general theorem (see [32]) which asserts that the cohomology of $B$ in $\mathrm{H}_{i}(\mathfrak{g}, U(\mathfrak{g}))$ for positively graded $\mathfrak{g}$ is trivial and generated by constants $\mathbb{C} \subset \mathrm{H}_{0}(\mathfrak{g}, U(\mathfrak{g}))$. The rest follows from the information about homology of $Y M$ with coefficients in $U(Y M)$ (see 133).

The proof of the statement that co-dimension of $\operatorname{Im}\left(A_{0}\right)$ in the space of $\mathfrak{s u s y}$ invariant elements in $\mathrm{H}_{0}(Y M, U(Y M))$ is equal to one easily follows from this lemma. We know that the image of map $A_{1}$ has co- dimension one in the space of $\mathfrak{s u s y}$-invariants. The operator $B$ preserves $\mathfrak{s o}(10) \ltimes \mathfrak{s u s y}$-invariant subspaces. If we write $\mathrm{H}_{0}(Y M, U(Y M))=\mathbb{C}+\underline{\mathrm{H}}_{0}(Y M, U(Y M))$, the operator $B$ admits the inverse: $B^{-1}: \mathrm{H}_{1}(Y M, U(Y M))^{\operatorname{Spin}(10)} \rightarrow \underline{\mathrm{H}}_{0}(Y M, U(Y M))^{\operatorname{Spin}(10)}$. The identity $A_{1} B=B A_{0}$ implies that $A_{0}$ is equal to $B^{-1} A_{1} B$, when restricted on $\underline{\mathrm{H}}_{0}(Y M, U(Y M))^{\mathrm{Spin}(10)}$. The claim follows from the corresponding statement for $A_{1}$.

The reader should consult for missing details the references [33] and [36].

We have analyzed the case of reduced SYM theory. Very similar considerations can be applied to the unreduced case.

First of all we should formulate the analog of Proposition 14 Let us notice that it follows from (35) that $W \otimes \mu_{-1} \subset L^{3}$, hence $W \subset L^{3} \otimes \mu_{1}$. Using (74) we conclude that there is an embedding $\mathcal{W} \rightarrow \mathcal{T Y M}^{\bullet}$, where we consider $\mathcal{W}$ as a graded vector bundle with one graded component in grading 3 that corresponds to $P$-module $W$ and has zero differential.

Proposition 20 The embedding of $\mathcal{W}$ into $\mathcal{T Y M}^{\bullet}$ is a quasi-isomorphism. 
The proof will be given in Appendix E

Using this proposition we can write down an exact sequence analogous to (76).

Corollary 21 There is a long exact sequence connecting $\mathrm{H}^{k}(L, U(T Y M)), \mathrm{H}_{k}(L, U(T Y M))$ and hypercohomology:

$$
\begin{aligned}
& \cdots \rightarrow \mathrm{H}_{3-i, a-8}\left(L, \operatorname{Sym}^{j}(T Y M)\right) \stackrel{\delta}{\rightarrow} \mathrm{H}^{i, a}\left(L, \operatorname{Sym}^{j}(T Y M)\right) \rightarrow \\
& \rightarrow \mathrm{H}^{i+a-3 j}\left(\mathcal{Q}, \Lambda^{j}(\mathcal{W})(3 j-a)\right) \stackrel{\iota}{\rightarrow} \mathrm{H}_{2-i, a-8}\left(L, \operatorname{Sym}^{j}(T Y M)\right) \rightarrow \ldots
\end{aligned}
$$

Again using Borel-Weil-Bott theorem we can calculate the cohomology of $\mathcal{Q}$ with coefficients in vector bundles that enter this sequence.

\section{Proposition 22}

$$
\begin{aligned}
& i \geq 0 \\
& \mathrm{H}^{0}(\mathcal{Q}, \mathcal{O}(i))=[0,0,0,0, i], \quad \mathrm{H}^{10}(\mathcal{Q}, \mathcal{O}(-8-i))=[0,0,0, i, 0], \\
& \mathrm{H}^{0}(\mathcal{Q}, \mathcal{W}(i+1))=[1,0,0,0, i], \quad \mathrm{H}^{10}(\mathcal{Q}, \mathcal{W}(-8-i))=[0,0,0, i, 1], \\
& \mathrm{H}^{0}\left(\mathcal{Q}, \Lambda^{2}(\mathcal{W})(2+i)\right)=[0,1,0,0, i], \quad \mathrm{H}^{10}\left(\mathcal{Q}, \Lambda^{2}(\mathcal{W})(-8-i)\right)=[0,0,1, i, 0], \\
& \mathrm{H}^{9}\left(\mathcal{Q}, \Lambda^{2}(\mathcal{W})(-6)\right)=[0,0,0,0,0] \\
& \mathrm{H}^{0}\left(\mathcal{Q}, \Lambda^{3}(\mathcal{W})(3+i)\right)=[0,0,1,0, i], \quad \mathrm{H}^{10}\left(\mathcal{Q}, \Lambda^{3}(\mathcal{W})(-7-i)\right)=[0,1,0, i, 0], \\
& \mathrm{H}^{1}\left(\mathcal{Q}, \Lambda^{3}(\mathcal{W})(1)\right)=[0,0,0,0,0] \\
& \mathrm{H}^{0}\left(\mathcal{Q}, \Lambda^{4}(\mathcal{W})(3+i)\right)=[0,0,0,1, i], \quad \mathrm{H}^{10}\left(\mathcal{Q}, \Lambda^{4}(\mathcal{W})(-6-i)\right)=[1,0,0, i, 0], \\
& \mathrm{H}^{0}\left(\mathcal{Q}, \Lambda^{5}(\mathcal{W})(3+i)\right)=[0,0,0,0, i], \quad \mathrm{H}^{10}\left(\mathcal{Q}, \Lambda^{5}(\mathcal{W})(-5-i)\right)=[0,0,0, i, 0],
\end{aligned}
$$

To analyze super Poincaré invariant deformations of unreduced theory we should study Spin(10)-invariant part of long exact sequence (79). As in reduced case Spin(10)-part of the exact sequence splits into short exact sequences. More precisely if the indices $(i, j, \boldsymbol{a})$ belong to the set $\{(3,0,8),(4,2,12),(6,5,20)\}$ then we have the splitting 


$$
\begin{aligned}
& 0 \rightarrow \mathrm{H}^{i+a-3 j-1}\left(\mathcal{Q}, \Lambda^{j}(\mathcal{W})(3 j-a)\right)^{\operatorname{Spin}(10)} \rightarrow \mathrm{H}_{3-i, a-8}\left(L, \operatorname{Sym}^{j}(T Y M)\right)^{\operatorname{Spin}(10)} \stackrel{\delta}{\rightarrow} \\
& \stackrel{\delta}{\rightarrow} \mathrm{H}^{i, a}\left(L, \operatorname{Sym}^{j}(T Y M)\right)^{\operatorname{Spin}(10)} \rightarrow 0 \\
& \quad \text { If }(i, j, \boldsymbol{a}) \in\{(0,0,0)(2,3,8)(3,5,12)\} \\
& 0 \rightarrow \mathrm{H}_{3-i, a-8}\left(L, \operatorname{Sym}^{j}(T Y M)\right)^{\operatorname{Spin}(10)} \stackrel{\delta}{\rightarrow} \mathrm{H}^{i, a}\left(L, \operatorname{Sym}^{j}(T Y M)\right)^{\operatorname{Spin}(10)} \rightarrow \\
& \rightarrow \mathrm{H}^{i+a-3 j}\left(\mathcal{Q}, \Lambda^{j}(\mathcal{W})(3 j-a)\right)^{\operatorname{Spin}(10)} \rightarrow 0
\end{aligned}
$$

and for all other $(i, j, \boldsymbol{a})$

$\mathrm{H}_{3-i, a-8}\left(L, \operatorname{Sym}^{j}(T Y M)\right)^{\operatorname{Spin}(10)} \stackrel{\delta}{\cong} \mathrm{H}^{i, a}\left(L, \operatorname{Sym}^{j}(T Y M)\right)^{\operatorname{Spin}(10)}$

The Spin(10)-invariant part of hypercohomology is six-dimensional, but only three-dimensional part of it, as the reader can see in (81), gives a contribution to the cohomology $\mathrm{H}^{0}, \mathrm{H}^{2}$ and $\mathrm{H}^{3}$. The contribution to $\mathrm{H}^{0}$ is not interesting; the contribution to $\mathrm{H}^{2}$ gives the deformation $\delta \mathcal{L}_{16}$ and the contribution to $\mathrm{H}^{3}$ is trivial at the level of infinitesimal deformations of equations of motion (but it gives a non-trivial deformation of $\mathrm{L}_{\infty}$ action of supersymmetry, hence the construction of Section 7 can give a non-trivial formal deformation).

The analogs of operators $A_{k}$ and $T_{k}$ can be defined in the situation at hand; again $A_{k}=T_{k}$.

The most technical part of the proof is hidden in the verification of the analog of Lemma 18

Lemma 23 The co-kernels of the maps $i_{* 1}: H_{1}(Y M, U(T Y M))^{\operatorname{Spin}(10)} \rightarrow$ $\mathrm{H}_{1}(L, U(T Y M))^{\operatorname{Spin}(10)}$ and $i_{2}^{*}: H^{2}(L, U(T Y M))^{\operatorname{Spin}(10)} \rightarrow \mathrm{H}^{2}(Y M, U(T Y M))^{\operatorname{Spin}(10)}$ have dimensions two and zero respectively.

The rest of the proof follows along the lines of the proof in reduced case. In particular one should use the analog of Lemma 19 


\section{The BV formalism: a geometric approach}

Our considerations will be based on the Batalin-Vilkovisky (BV) formalism. In this formalism a classical system is represented by an action functional $S$ defined on an odd symplectic manifold $M$ and obeying the classical Master equation

$$
\{S, S\}=0 .
$$

where $\{\cdot, \cdot\}$ stands for the odd Poisson bracket. Using an odd symplectic form $\omega=d x^{i} \omega_{i j} d x^{j}$ we assign to every even functional $F$ an odd vector field $\xi=\xi_{F}$ defined by the formula

$$
\xi^{i} \omega_{i j}=\frac{\partial F}{\partial z^{j}}
$$

(Hamiltonian vector field corresponding to the functional $F$ ). The form $\omega$ is invariant with respect to $\xi_{F}$. In particular we may consider an odd vector field $Q=\xi_{S}$; this field obeys $[Q, Q]=0$. Here $[\cdot, \cdot]$ stands for supercommutator. The solutions to the equations of motion (EM) are identified with zero locus of $Q$.

In an equivalent formulation of BV we start with an odd vector field $Q$ obeying $[Q, Q]=0$. We require the existence of $Q$-invariant odd non-degenerate closed two- form $\omega$ (odd symplectic form). Then we can restore the action functional from $Q^{i} \omega_{i j}=\frac{\partial S}{\partial z^{j}}$.

Sometimes is convenient to drop the condition of non-degeneracy of the form $\omega$. In this case one cannot define the Poisson bracket, but the definition of Hamiltonian vector field still makes sense.

We say that in BV-formalism a classical system is defined by means of an odd vector field $Q$ obeying $[Q, Q]=0$. In geometric language we are saying that a classical system is a $Q$-manifold. Fixing a vector field $Q$ we specify equations of motion of our system, but we do not require that EM come from an action functional. If there exists a $Q$-invariant odd symplectic form we can say that our system comes from action functional $S$ obeying classical Master equation $\{S, S\}=0$. In this case we say that we are dealing with a Lagrangian system. In geometric language we can identify it with an odd symplectic $Q$-manifold.

Infinitesimal deformation of a classical system corresponds to a vector field $\xi$ 
obeying $[Q, \xi]=0$ (then $[Q+\xi, Q+\xi]=0$ in the first order with respect to $\xi)$. An infinitesimal deformation $\xi$ is trivial if $\xi=[Q, \eta]$ because such a deformation corresponds to a change of variables (field redefinition) $x^{i} \rightarrow x^{i}+\eta^{i}$. The operator $\xi \rightarrow[Q, \xi]$ defines a cohomological differential on $\operatorname{Vect}(M)$ which we by abuse of notations denote by the same letter $Q$. The deformations of a classical system corresponding to vector field $Q$ are labeled by $Q$-cohomology of the space of vector fields $V e c t(M)$.

The algebra of smooth functions $C^{\infty}(M)$ on $M$ can be considered as super commutative differential graded algebra with differential $Q^{i} \frac{\partial}{\partial x^{i}}$, which we as in case of the space of vector fields denote by $Q$. The cohomology $\mathrm{H}\left(C^{\infty}(M), Q\right)$ can be identified with classical observables. In other words a classical observable is defined as a function $O$ obeying $Q(O)=0$. Two classical observables $O_{1}, O_{2}$ are identified if $O_{1}-O_{2}=Q\left(O^{\prime}\right)$ for some $O^{\prime} \in C^{\infty}(M)$. Classical observables label infinitesimal deformations of solutions to the classical master equation which are the same as deformations of classical Lagrangian system in BV formalism. This follows from the remark that the equation $\{S+\sigma, S+\sigma\}=0$ where $S$ is the solution of master equation and $\sigma$ is infinitesimally small is equivalent to the equation $Q(\sigma)=0$.

In the space $S o l$ of solutions to EM (in the zero locus of $Q$ ) we should identify solutions $x$ with $x+\delta x$ where $\delta x^{i}=Q^{i}(x+\delta)-Q^{i}(x)$, where $\delta$ is infinitesimally small. The space obtained by means of this identification is denoted by $\mathrm{Sol} / \sim$. 16

A classical system has many equivalent descriptions in BV-formalism. The simplest way to see this is to notice that a system with coordinates $\left(y^{1}, \ldots, y^{n}, \xi_{1}, \ldots, \xi_{n}\right)$, symplectic form $d y^{i} d \xi_{i}$ and action functional $a_{i j} y^{i} y^{j}$ is physically trivial. Here $\xi_{i}$ and $y^{i}$ have opposite parities and the matrix $a_{i j}$ is nondegenerate.

\footnotetext{
${ }^{16}$ More geometrically we can say that on the zero locus $\operatorname{Sol}$ of $Q$ there exists a foliation $\mathcal{F}_{Q}$. The tangent vectors to $S o l$ can be identified with the kernel $\operatorname{Ker} \frac{\delta Q^{i}}{\delta x^{j}}$ of $Q$; the leaves of $\mathcal{F}_{Q}$ are tangent to the image $\operatorname{Im} \frac{\delta Q^{i}}{\delta z^{j}}$. We identify two solutions belonging to the same leaf, hence $\mathrm{Sol} / \sim$ can be considered as the space of leaves of the foliation. Notice that in most cases the foliation on the space of solutions is singular (the dimension of the kernel varies).
} 
Consider two Q-manifolds $(M, Q)$ and $\left(M^{\prime}, Q^{\prime}\right)$. A map $f: M \rightarrow M^{\prime}$ is called a $Q$-map if it agrees with action of $Q$ 's (i.e. $Q f^{*}=f^{*} Q$ where $f^{*}$ is the homomorphism $C^{\infty}\left(M^{\prime}\right) \rightarrow C^{\infty}(M)$ induced by the map $\left.f\right)$. Such a map induces a map of observables (a homomorphism of cohomology groups $\left.\mathrm{H}\left(C^{\infty}\left(M^{\prime}\right), Q^{\prime}\right) \rightarrow \mathrm{H}\left(C^{\infty}(M), Q\right)\right)$. If $f$ defines an isomorphism between spaces of observables we say that $f$ is a quasi-isomorphism. Under some additional requirements this isomorphism implies isomorphism of spaces of solutions $\mathrm{Sol} / \sim$. Quasi-isomorphism should be considered as isomorphism of classical physical systems. However for Lagrangian systems one should modify the definition of physical equivalence, requiring that quasi-isomorphism is compatible with symplectic structure in some sense.

Let us consider the Taylor series decomposition

$$
Q^{a}(x)=\sum_{b_{1}, \ldots, b_{n}} Q_{b_{1}, \ldots, b_{n}}^{a} x_{1}^{b_{1}} \ldots x_{n}^{b_{n}}
$$

of the coefficients of the vector field $Q=\sum Q^{a} \frac{\partial}{\partial x^{a}}$ in the neighborhood of the critical point. Here $x^{i}$ are local coordinates in the patch, the critical point is located at $x=0$. The coefficient $Q_{b_{1}, \ldots, b_{n}}^{a}$ of this expansion specifies an algebraic $n$-ary operation $\psi_{n}\left(s_{1}, \ldots, s_{n}\right)$ on $\Pi T_{0}$ (on the tangent space with reversed parity at $x=0)$. A set of some quadratic relations on $\psi_{n}$ is a corollary of Master equation $[Q, Q]=0$. A collection $\left\{\psi_{n}\right\}_{n=1}^{\infty}$ that satisfies these quadratic relations specify a structure of $\mathrm{L}_{\infty}$ algebra on $T_{0}$ (see Appendix $\mathrm{A}$ for more detail). One can say that $\mathrm{L}_{\infty}$ algebra is a formal $Q$-manifold 17

In the case when the only nonzero coefficients are $Q_{b}^{a}$ and $Q_{b_{1}, b_{2}}^{a}$ the corresponding $\mathrm{L}_{\infty}$ algebra can be identified with a differential graded Lie algebra. The tensor $Q_{b}^{a}$ corresponds to the differential and $Q_{b_{1}, b_{2}}^{a}$ to the bracket.

An $\mathrm{L}_{\infty}$ homomorphism of $\mathrm{L}_{\infty}$ algebras is defined as a $Q$-equivariant map

\footnotetext{
${ }^{17}$ We define a formal manifold saying that functions on it are power series with respect to $n$ commuting and $m$ anticommuting variables. Notice that this definition is not standard; other terms for the same notion are "germ of a manifold" and "infinitesimal manifold". From the other side Sullivan suggested to use the term "formal manifold" in completely different setting.
} 
between the corresponding formal $Q$-manifolds. We can use this notion to define an $\mathrm{L}_{\infty}$ action of a Lie algebra on a $Q$-manifold $M$. Conventional action of a Lie algebra is a homomorphism of this Lie algebra into Lie algebra $\operatorname{Vect}(M)$ of vector fields on $M . \mathrm{L}_{\infty}$ action of a Lie algebra $\mathfrak{g}$ on $(M, Q)$ is an $\mathrm{L}_{\infty}$ homomorphism of $\mathfrak{g}$ to the differential graded algebra $(\operatorname{Vect}(M), Q)$. This definition is rather inexplicit to say the least. A more direct definition shall be introduced presently.

The action of a Lie algebra is specified by vector fields $q_{\alpha}$, corresponding to generators $e_{\alpha}$ of $\mathfrak{g}$. The generators obey relations $\left[e_{\alpha}, e_{\beta}\right]=f_{\alpha \beta}^{\gamma} e_{\gamma}$, where $f_{\alpha \beta}^{\gamma}$ are the structure constants of $\mathfrak{g}$ in the basis $e_{\alpha}$. A weak action of $\mathfrak{g}$ requires that this relation is valid up to $Q$-exact terms:

$$
\left[q_{\alpha}, q_{\beta}\right]=f_{\alpha \beta}^{\gamma} q_{\gamma}+\left[Q, q_{\alpha \beta}\right]
$$

Even for a weak action we have a genuine Lie algebra action on observables and on $\mathrm{Sol} / \sim$.

To define an $\mathrm{L}_{\infty}$ action of Lie algebra $\mathfrak{g}$ we need not only $q_{\alpha}, q_{\alpha \beta}$, but also their higher analogs $q_{\alpha_{1} \ldots \alpha_{i}}$ obeying the relations similar to (84). This can be formalised as follows. One can consider $q_{\alpha_{1} \ldots \alpha_{i}}$ as components of linear maps

$$
q^{i}: \operatorname{Sym}^{i}(\Pi \mathfrak{g}) \rightarrow \Pi V e c t(M)
$$

They can be assembled into a vector field $q$ on $\Pi \mathfrak{g} \times M$. A choice of a basis in $\mathfrak{g}$ defines coordinates on $\Pi \mathfrak{g}$. In such coordinates the $i$-th Taylor coefficient coincides with the map $q^{i}$. The coordinates $c^{\alpha}$ on $\Pi \mathfrak{g}$ can be identified with ghost variables of the Lie algebra $\mathfrak{g}$. One can consider $q$ as a vector field on $M$ depending on ghost variables.

Let us introduce a super-commutative differential algebra $C^{\bullet}(\mathfrak{g})$ as the algebra of polynomial functions in ghost variables $c^{\alpha}$ with the differential

$$
d_{\mathfrak{g}}=\frac{1}{2} f_{\alpha \beta}^{\gamma} c^{\alpha} c^{\beta} \frac{\partial}{\partial c^{\gamma}} .
$$

Odd ghosts correspond to even generators, even ghosts correspond to odd generators. The Lie group cohomology with trivial coefficients is defined as the 
cohomology of $d_{\mathfrak{g}}$.

The collection (85) defines a $\mathrm{L}_{\infty}$ action if the ghost dependent vector field $q$ satisfies

$$
d_{\mathfrak{g}} q+[Q, q]+\frac{1}{2}[q, q]=0 .
$$

Notice, that instead of $q$ we can consider ghost dependent vector field $\tilde{q}=$ $Q+q ;$ in terms of this field (165) takes the form

$$
d_{\mathfrak{g}} \tilde{q}+\frac{1}{2}[\tilde{q}, \tilde{q}]=0
$$

The notion of $\mathrm{L}_{\infty}$ action is a particular case of the notion of $\mathrm{L}_{\infty}$ module. Recall that a $\mathfrak{g}$-module where $\mathfrak{g}$ is a Lie algebra can be defined as as a homomorphism of $\mathfrak{g}$ in the Lie algebra of linear operators acting on vector space $N$. (In other words a $\mathfrak{g}$-module is the same as linear representation of $\mathfrak{g}$.) If $N$ is a complex the space of linear operators on $N$ is a differential Lie algebra. A structure of $\mathrm{L}_{\infty} \mathfrak{g}$ module on $N$ is an $\mathrm{L}_{\infty}$ homomorphism of $\mathfrak{g}$ into this differential Lie algebra. This structure can be described as a polynomial function $q$ of ghosts $c^{\alpha}$ taking values in the space of linear operators on $N$ and obeying relation:

$$
d_{\mathfrak{g}} q+[Q, q]+\frac{1}{2}[q, q]=0 .
$$

As usual we denote by $Q$ the differential in $N$ (cf. (165)).

We can define cohomology $\mathrm{H}_{\mathfrak{g}}^{\bullet}(N)=\mathrm{H}^{\bullet}(\mathfrak{g}, N)$ of the Lie algebra $\mathfrak{g}$ with coefficients in $\mathrm{L}_{\infty} \mathfrak{g}$-module $N$ to be the cohomology of the differential

$$
d_{c}=d_{\mathfrak{g}}+q+Q=\frac{1}{2} f_{\alpha \beta}^{\gamma} c^{\alpha} c^{\beta} \frac{\partial}{\partial c^{\gamma}}+\sum_{k} \frac{1}{k !} q_{\alpha_{1}, \ldots, \alpha_{k}} c^{\alpha_{1}} \cdots c^{\alpha_{k}}+Q .
$$

acting on the space of $N$-valued functions of ghosts (i.e. on the tensor product $\left.C^{\bullet}(\mathfrak{g}) \otimes N\right)$. It follows immediately from (89) that $d_{c}$ is a differential. Conversely, if the expression (90) is a differential then $q$ specifies an $\mathrm{L}_{\infty}$ action.

To define homology of the Lie algebra $\mathfrak{g}$ with coefficients in $\mathrm{L}_{\infty}$ module $N$ we use the differential $d_{h}$ acting on $N$-valued polynomial functions of ghost variables $c_{\alpha}$ (on the tensor product $\mathrm{Sym} \Pi \mathfrak{g} \otimes N$ ). This differential can be obtained from 
$d_{c}$ by means of formal Fourier transform in ghost variables, i.e. a substitution of the derivation with respect to $c_{\alpha}$ instead of multiplication by $c^{\alpha}$ and of the multiplication by $c_{\alpha}$ instead of derivation with respect to $c^{\alpha}$ :

$$
d_{h}=\frac{1}{2} f_{\alpha \beta}^{\gamma} c_{\gamma} \frac{\partial}{\partial c_{\alpha}} \frac{\partial}{\partial c_{\beta}}+\sum_{k} \frac{1}{k !} q_{\alpha_{1}, \ldots, \alpha_{k}} \frac{\partial}{\partial c_{\alpha_{1}}} \cdots \frac{\partial}{\partial c_{\alpha_{k}}}+Q
$$

In light of this discussion the definition of a Hamiltonian $\mathrm{L}_{\infty}$ action on odd symplectic manifold $M$ is obvious. In the formula (165) we replace the vector field $q$ by a function and the commutator by the Poisson bracket. A Hamiltonian $\mathrm{L}_{\infty}$ symmetry of classical BV action functional $S$ can be specified by a function of ghosts and fields (i.e. by an element $\sigma \in C^{\bullet}(\mathfrak{g}) \otimes C^{\infty}(M)$ ). This element should obey the equation

$$
d_{\mathfrak{g}} \sigma+\{S, \sigma\}+\frac{1}{2}\{\sigma, \sigma\}=0 .
$$

Introducing a function $\hat{S}=\sigma+S$ we can rewrite (92) in the form

$$
d_{\mathfrak{g}} \hat{S}+\frac{1}{2}\{\hat{S}, \hat{S}\}=0
$$

Mathematically it is very natural to let both ingredients of a BV package $(Q, \omega)$ to be ghost dependent. Such ghost dependent pair $(\hat{Q}, \hat{\omega})$ satisfies a block of axions and give rise to constructions analogous to ghost independent setup:

$$
\hat{Q}^{i} \hat{\omega}_{i j}=\frac{\partial \hat{S}}{\partial x^{i}}
$$

In this formula ghost variables are parameters. The condition $Q \omega=0$ extends to the condition $d_{c} \hat{\omega}=0$ where in $q_{\alpha_{1}, \ldots, \alpha_{k}}$ and $Q$ in (90) act on $\hat{\omega}$ by Lie derivatives; the operator $d_{\mathfrak{g}}$ acts only on ghost parameters. In addition we assume that ghost coefficients of $\hat{\omega}$ are de Rham closed and and ghost independent term is nondegenerate. We shall call ghost dependent two-forms, that satisfy last two conditions closed and nondegenerate respectively.

In many interesting situations an action of a Lie algebra on shell (on the space $S o l / \sim)$ can be lifted to an $\mathrm{L}_{\infty}$ action off shell. Conversely any $\mathrm{L}_{\infty}$ action of Lie algebra (or, more generally, any weak action) on a Q-manifold (off- shell action) generates ordinary Lie algebra action on shell. 
18

Let us come back to the general theory of deformations in BV-formalism. Recall that infinitesimal deformations of solution to the classical Master equation (82) (of classical Lagrangian system in BV formalism) are labeled by observables (by cohomology $\mathrm{H}\left(C^{\infty}(M), Q\right)$ of $Q$ in the space $C^{\infty}(M)$ ). Every deformation of action $S$ induces a deformation of $Q$ and we have a homomorphism of corresponding cohomology groups $\mathrm{H}\left(C^{\infty}(M), Q\right) \rightarrow \mathrm{H}(V e c t(M), Q)$.

Let us analyze classification of infinitesimal deformations of a classical system compatible with symmetries of the system. We assume that the system is described by an odd vector field $Q$ obeying $[Q, Q]=0$ on a supermanifold $M$ and the Lie algebra of symmetries $\mathfrak{g}$ acts in $\mathrm{L}_{\infty}$ fashion on $M$. Then the complex $(V \operatorname{ect}(M), Q)$ as $\mathrm{L}_{\infty} \mathfrak{g}$-module. We would like to deform simultaneously the vector field $Q$ and the $\mathrm{L}_{\infty}$ action specified by the ghost dependent vector field $q$. We shall show that the infinitesimal deformations are classified by elements of cohomology $\mathrm{H}^{\bullet}(\mathfrak{g},(\operatorname{Vect}(M), Q))$ of Lie algebra $\mathfrak{g}$ with coefficients in differential $L_{\infty} \mathfrak{g}$-module $(\operatorname{Vect}(M), Q)$. To prove this statement we notice that $Q$ and $q$ are combined in the ghost dependent vector field $\tilde{q}$, hence the deformation we are interested in can be considered as the deformation of $\tilde{q}$ that preserves the relation (88). In other words this deformation should obey

$$
d_{\mathfrak{g}} \delta \tilde{q}+[\tilde{q}, \delta \tilde{q}]=0
$$

This condition means that $\delta \tilde{q}$ is a $d_{c}$-cocycle (90)). It is easy to see that cohomologous cocycles specify equivalent deformations and $\mathrm{H}^{\bullet}(\mathfrak{g},(\operatorname{Vect}(M), Q))$ is in one-to-one correspondence with the infinitesimal deformations of our objects.

\footnotetext{
${ }^{18}$ More generally, we can consider $\mathrm{L}_{\infty} \mathfrak{g}$-module $N$. We say that the structure of $\mathrm{L}_{\infty}$ module on $N$ is Hamiltonian if $N$ can be equipped with $\mathfrak{g}$-invariant inner product. (We say that the inner product is $\mathfrak{g}$-invariant if $q$ specifying $\mathrm{L}_{\infty} \mathfrak{g}$-action takes values in the Lie algebra of linear operators on $N$ that preserve the inner product.) If we have an odd symplectic $Q$ - manifold $M$ we can take as $N$ the $\mathrm{L}_{\infty}$ algebra constructed as the Taylor decomposition of $Q$ in Darboux coordinates in the neighborhood of a point belonging to the zero locus of $Q$. This algebra is equipped with odd inner product coming from the odd symplectic form. A Hamiltonian $\mathrm{L}_{\infty}$ action on $M$ generates a Hamiltonian $\mathrm{L}_{\infty}$ action on $N$.
} 
It is important to emphasize that commutation relations of the new symmetry generators are deformed (even if we have started with genuine action of $\mathfrak{g}$ we can obtain a weak action after the deformation). Nevertheless on shell, i.e. after restriction to $\mathrm{Sol} / \sim$, commutation relations do not change.

As we have said the cohomology $\mathrm{H}^{\bullet}(\mathfrak{g},(\operatorname{Vect}(M), Q))$ describes $\mathrm{L}_{\infty}$ deformations of $Q$ and $\mathrm{L}_{\infty}$ action. Notice, that two different $\mathrm{L}_{\infty}$ actions can induce the same Lie algebra action on shell. It is easy to see that only the ghost number one components enter in the expressions for generators of Lie algebra symmetries on shell.

It is important to emphasize that analyzing deformations in BV formulation we can chose any of physically equivalent classical systems ( the cohomology we should calculate is invariant with respect to quasi-isomorphism).

We have analyzed the deformations of $Q$ (of equations of motion) preserving the symmetry. Very similar consideration can be applied in Lagrangian formalism. In this case we start with the functional $\hat{S}=\sigma+S$ combining the classical BV functional $S$ and Hamiltonian $\mathrm{L}_{\infty}$ symmetry. We should deform this functional preserving the relation (93). We keep symplectic form undeformed. We see that that the infinitesimal deformation obeys

$$
d_{\mathfrak{g}} \delta \hat{S}+\{\hat{S}, \delta \hat{S}\}=0
$$

Interpreting this equation as a cocycle condition we obtain the following statement.

Proposition 24 Let us consider a BV action functional $S$ on an odd symplectic manifold $M$ together with Hamiltonian $L_{\infty}$ action of Lie algebra $\mathfrak{g}$ (i.e. with a functional $\sigma$ of fields and ghosts such that $\hat{S}=\sigma+S$ obeys (93)). Then the algebra $C^{\infty}(M)$ can be considered as a differential $L_{\infty} \mathfrak{g}$-module (the differential is defined as a Poisson bracket with $S$ ). Infinitesimal deformations of $B V$ action functional and Hamiltonian $L_{\infty}$ action are governed by the Lie algebra cohomology of $\mathfrak{g}$ with coefficients in this module. 
Proposition 25 Let us consider a supermanifold $M$ together with an odd vector field $Q$ obeying $[Q, Q]=0$ and with $L_{\infty}$ action of a Lie algebra $\mathfrak{g}$ on $(M, Q)$ (in other words we have a classical system with symmetry Lie algebra $\mathfrak{g}$ ). Let us assume that the manifold $M$ is equipped with a non-degenerate ghost-dependent closed two-form $\hat{\omega}$ that is compatible with the $L_{\infty}$ action(i.e. $d_{c} \hat{\omega}=0$ ). Then the algebra $C^{\infty}(M)$ is an $L_{\infty} \mathfrak{g}$-module. The infinitesimal deformations of $Q$ together with Hamiltonian $L_{\infty}$ action preserving $\hat{\omega}$ are governed by the Lie algebra cohomology of $\mathfrak{g}$ with coefficients in this module.

Last two propositions are related because all nondegenerate ghost-dependent closed odd two-forms are diffeomorphic, if we allow ghost-dependent diffeomorphisms. Such diffeomorphism can be used to eliminate ghost dependence of the form.

Our experience with pure spinor formalism dictates a need for extension of above theorem to manifolds with a degenerate two-form. As in the strictly symplectic variant of this definition every hamiltonian vector field $\xi$ ( a vector field preserving the form $\omega$ ) defines a Hamiltonian $F$ by the formula (83) (of course, the Hamiltonian is defined up to an additive constant).This construction of the Hamiltonian defines a linear map from the algebra of hamiltonian vector fields $H a m$ to the space of functions $C^{\infty}(M) / \mathbb{C}$ :

$$
H: H a m \rightarrow C^{\infty}(M) / \mathbb{C}
$$

For degenerate form $\omega$ the map $H$ is not invertible and we do not expect to have a Poisson structure on the algebra of functions. The commutator with $Q$ defines a differential on Ham. Infinitesimal deformations of $Q$ are in bijections with cohomology classes of the complex $(\operatorname{Ham}, Q)$. A closed two-form $\omega$ is homologically non-degenerate if it defines a nondegenerate pairing on cohomology $\mathrm{H}\left(T_{x}, Q\right)$ of the tangent complex $\left(T_{x}, Q\right)$ at each critical point $x$ of $\left.Q\right)$. In this case we expect that $H$ is a quasiisomorphism (i.e. it induces an isomorphism $\mathrm{H}(\operatorname{Ham}, Q) \rightarrow \mathrm{H}\left(C^{\infty}(M) / \mathbb{C}, Q\right)$, which enables us to transfer the Lie algebra structure onto $\left.\mathrm{H}\left(C^{\infty}(M) / \mathbb{C}, Q\right)\right)$. The reason to believe in this statement is 
a validity of a local statement at a critical point $x$ of $Q$. More precisely, the statement is correct if we replace $M$ by a germ of $M$ at a critical point . As it has already been pointed out this framework is equivalent to the framework of $L_{\infty}$-algebras with odd inner product). Hence in homologically non-degenerate case deformations are classified by $\mathrm{H}\left(C^{\infty}(M)\right)$ (at least for formal $Q$-manifolds).

These statements can be generalized to a $\mathfrak{g}$-equivariant setup . We say that a two-form $\hat{\omega}$ homologically non-degenerate if its ghost-free term is homologically non-degenerate. The proposition 25 is still valid if the closed two-form $\hat{\omega}$ on a formal $Q$-manifold is homologically non-degenerate. Identifying formal $Q$ manifolds with $L_{\infty}$-algebras we can use proposition 25 to classify deformations of $L_{\infty}$ algebras with homologically non-degenerate odd inner product (i.e. with inner product that is non-degenerate on homology).

Let us describe some formulations of 10D SUSY YM in BV formalism. For simplicity we shall restrict ourselves to the theory reduced to a point.

In component formalism besides fields $A_{i}, \chi^{\alpha}$, antifields $A_{i}^{*}, \chi_{\alpha}^{*}$ we have ghosts $c$ and anti-fields for ghosts $c^{*}$ (all of them are $n \times n$ matrices). The $\mathrm{BV}$ action functional has the form

$$
\mathcal{L}_{B V S Y M}=\operatorname{tr}\left(\frac{1}{4} F_{i j} F_{i j}+\frac{1}{2} \Gamma_{\alpha \beta}^{i} \chi^{\alpha} \nabla_{i} \chi^{\beta}+\nabla_{i} c A_{i}^{*}+\chi^{\alpha}\left[c, \chi_{\alpha}^{*}\right]+\frac{1}{2}[c, c] c^{*}\right)
$$

The corresponding vector field $Q$ is given

$$
\begin{aligned}
& Q\left(A_{i}\right)=-\nabla_{i} c \\
& Q\left(\psi^{\alpha}\right)=\left[c, \psi^{\alpha}\right] \\
& Q(c)=\frac{1}{2}[c, c] \\
& Q\left(c^{*}\right)=\sum_{i=1}^{10} \nabla_{i} A^{* i}+\sum_{\alpha}\left[\psi^{\alpha}, \psi_{\alpha}^{*}\right]+\left[c, c^{*}\right] \\
& Q\left(A^{* m}\right)=-\sum_{i=1}^{10} \nabla_{i} F_{i m}+\frac{1}{2} \sum_{\alpha \beta} \Gamma_{\alpha \beta}^{m}\left[\psi^{\alpha}, \psi^{\beta}\right]-\left[c, A^{* m}\right] \\
& Q\left(\psi_{\alpha}^{*}\right)=-\sum_{i=1}^{10} \sum_{\beta} \Gamma_{\alpha \beta}^{i} \nabla_{i} \psi^{\beta}-\left[c, \psi_{\alpha}^{*}\right]
\end{aligned}
$$

Another possibility is to work in the formalism of pure spinors. 
Let $\mathrm{S}=\mathbb{C}^{16}$ be a 16 -dimensional complex vector space with coordinates $\lambda^{1}, \ldots, \lambda^{16}$. Denote by $\mathcal{C}$ a cone of pure spinors in $\mathrm{S}$ defined by equation

$$
\Gamma_{\alpha \beta}^{i} \lambda^{\alpha} \lambda^{\beta}=0
$$

and by $\mathcal{S}=\mathbb{C}\left[\lambda^{1}, \ldots, \lambda^{16}\right] / \Gamma_{\alpha \beta}^{i} \lambda^{\alpha} \lambda^{\beta}$ the space of polynomial functions on $\mathcal{C}$.

The fields in this formulation are elements $A(\lambda, \theta) \in \mathcal{S} \otimes \Lambda\left[\theta^{1}, \ldots, \theta^{16}\right] \otimes$ Mat $_{N}$; they can be considered as Mat ${ }_{N}$-valued polynomial super-functions on $C \mathcal{Q} \times \Pi S$. We define differential $d$ acting on these fields by the formula $d=$ $\lambda^{\alpha} \frac{\partial}{\partial \theta^{\alpha}}$. Using the terminology of Section 2.1 we can identify the space of fields with tensor product of reduced Berkovits algebra $B_{0}$ and Mat $_{N}$.

The vector field $Q$ on the space of fields is given by the formula

$$
\delta_{Q} A=d A+\frac{1}{2}\{A, A\} .
$$

This vector field specifies a classical system quasi-isomorphic to the classical system corresponding to the action functional ( 96) (see [34]).

An odd two- form on the space of fields is given by the formula:

$$
\omega\left(\delta A_{1}, \delta A_{2}\right)=\operatorname{tr}\left(\delta A_{1} \delta A_{2}\right)
$$

The trace tr is nontrivial only on $\mathcal{S}_{3} \otimes \Lambda^{5}\left[\theta^{1}, \ldots, \theta^{16}\right]$. Denote $\Gamma$ be the only $\operatorname{Spin}(10)$ invariant element in $\mathcal{S}_{3} \otimes \Lambda^{5}\left[\theta^{1}, \ldots, \theta^{16}\right]$. Let $p$ be the only $\operatorname{Spin}(10)$ invariant projection on the span $\langle\Gamma\rangle$. Then $p(a)=\operatorname{tr}(a) \Gamma$. This definition fixes tr up to a constant. (The trace at hand was introduced in [4, where more explicit formula was given.) Notice that $\omega$ is a degenerate closed two-form. However, it is homologically nondegenerate; this allows us to study infinitesimal Lagrangian deformations using a generalization of proposition 25 ,

In BV-formalism equations of motion can be obtained from the action functional

$$
S(A)=\operatorname{tr}\left(A d A+\frac{2}{3} A^{3}\right),
$$

obeying the classical Master equation $\{S, S\}=0$ (recall that we factorize the space of fields with respect to Ker $\omega$ and $S$ descends to the quotient). The vector field $Q$ specified by the formula (99) corresponds to this action functional. 
The BV formulation of unreduced SYM theory in terms of pure spinors is similar. The basic field $A(x, \lambda, \theta)$ where $x$ is a ten-dimensional vector is matrixvalued. The differential $d$ is defined by the formula $d=\lambda^{\alpha}\left(\frac{\partial}{\partial \theta^{\alpha}}+\Gamma_{\alpha \beta}^{i} \theta^{\beta} \frac{\partial}{\partial x^{i}}\right)$. In the terminology of Section 2.1 the space of fields is a tensor product of Berkovits algebra $B$ and Mat $_{N}$. The expressions for action functional and odd symplectic form remain the same, but tr includes integration over ten-dimensional space.

19

Notice that that in component version of BV formalism the standard supersymmetry algebra acts on shell, but off shell we have weak action of this algebra (commutation relations are satisfied up to $Q$-trivial terms). In pure spinor formalism we have genuine action of supersymmetry algebra, but the form $\omega$ is not invariant with respect to supersymmetry transformations. (However, the corrections to this form are $Q$-trivial.)

We shall show that the weak action of supersymmetry algebra can be extended to $\mathrm{L}_{\infty}$ action (Appendix $\mathrm{C}$ ). Moreover, in Appendix D we shall prove that for appropriate choice of this action it will be compatible with odd symplectic structure.

Let us apply our general considerations to 10D SUSY YM reduced to a point. In Section 3 we described SUSY deformations of this action functional in component formalism. Now we shall rewrite these deformations in BV formalism. Moreover, we shall be able to write down also the deformed supersymmetry.

Let us start with BV description of the theory based on the Lagrangian (96). A vector field $\xi$ on the underlying space is completely characterized by

\footnotetext{
${ }^{19}$ To establish the relation to the superspace formalism we recall that in $(10 \mid 16)$ dimensional superspace $\left(x^{n}, \theta^{\alpha}\right)$ SYM equations together with constraints can be represented in the form$$
F_{\alpha \beta}=0
$$

where $F_{\alpha \beta}=\left\{\nabla_{\alpha}, \nabla_{\beta}\right\}-\Gamma_{\alpha \beta}^{i} \nabla_{i}, \nabla_{\alpha}=D_{\alpha}+A_{\alpha}, D_{\alpha}=\frac{\partial}{\partial \theta^{\alpha}}+\Gamma_{\alpha \beta}^{i} \theta^{\beta} \frac{\partial}{\partial x^{i}}$. It follows from these equations that the covariant derivatives $\nabla(\lambda)=\lambda^{\alpha} \nabla_{\alpha}$ obey $[\nabla(\lambda), \nabla(\lambda)]=0$ if $\lambda$ is a pure spinor. This allows us interpret Yang-Mills fields as degree one components of $A(x, \theta, \lambda)$. Degree zero components of $A(x, \theta, \lambda)$ correspond to ghosts. Degree two components correspond to antifields, degree three to antifields for ghosts. Components of higher degree belong to the kernel of $\omega$ and can be disregarded (see [4] and [34 for detail).
} 
the values on the generators of the algebra of functions. We shall refer to these values as to components. As the generators can be naturally combined in matrices, the components of the vector fields are also matrix-valued. The vector fields of sypersymmetries $\theta_{\alpha}$ in the matrix space description have the following components (we omit matrix indices ):

$$
\begin{gathered}
\theta_{\alpha} A^{i}=\Gamma_{\alpha \beta}^{i} \chi^{\beta} \\
\theta_{\alpha} \chi^{\beta}=\Gamma_{\alpha}^{\beta i j}\left[A_{i}, A_{j}\right] .
\end{gathered}
$$

The component description of the vector fields $D_{i}$ and $G_{\alpha}$ is

$$
D_{i} A_{j}=\left[A_{i}, A_{j}\right], \quad D_{i} \chi^{\alpha}=\left[A_{i}, \chi^{\alpha}\right]
$$

and

$$
G_{\alpha} A_{j}=\left[\chi_{\alpha}, A_{j}\right], \quad G^{\alpha} \chi^{\beta}=\left[\chi^{\alpha}, \chi^{\beta}\right] .
$$

In this setup we have the identities

$$
\begin{aligned}
& {\left[\theta_{\alpha}, \theta_{\beta}\right]-\Gamma_{\alpha \beta} D_{i}=\left[Q, \eta_{\alpha \beta}\right],} \\
& {\left[\theta_{\alpha}, D_{i}\right]-\Gamma_{\alpha \beta i} G_{\chi^{\beta}}=\left[Q, \eta_{\alpha i}\right]}
\end{aligned}
$$

Here

$$
\begin{gathered}
\eta_{\alpha \beta} \chi^{\gamma}=2 P_{\alpha \beta}^{\gamma \delta} \chi_{\delta}^{*}, \\
\eta_{\alpha i} A_{j}=C_{\alpha i}^{\beta j} \chi_{\beta}^{*}, \quad \eta_{\alpha i} \chi^{\beta}=-C_{\alpha i}^{\beta j} A_{j}^{*}
\end{gathered}
$$

The tensors $P_{\alpha \beta}^{\gamma \delta}$ and $C_{\alpha i}^{\beta j}$ are proportional to $\Gamma_{\alpha \beta}^{i_{1}, \ldots, i_{5}} \Gamma_{i_{1}, \ldots, i_{5}}^{\gamma \delta}$ and to $\Gamma_{\alpha i}^{\beta j}$ respectively. We have described in Section 3 an infinite family of SUSY deformations (56). It is easy to write down the terms $q$ and $q_{\alpha}$ in the corresponding cocycle. It is obvious that $q$ is a Hamiltonian vector field corresponding to the functional $\delta \mathcal{L}$ given by the formula (56). To find the functional $\sigma_{\alpha}$ generating the Hamiltonian vector field $q_{\alpha} c^{\alpha}$ we should calculate $\theta_{\alpha} \delta \mathcal{L}$ and use (23). The calculation of $\theta_{\alpha} \delta \mathcal{L}$ repeats the proof of the supersymmetry of the deformation 
(9) and leads to the following result:

$$
\begin{aligned}
& \theta_{\alpha} \delta \mathcal{L}=\operatorname{tr}\left(\theta_{\alpha} \theta_{1} \ldots \theta_{16} G\right)= \\
& \operatorname{tr}\left(\sum_{\gamma=1}^{\alpha-1}(-1)^{\gamma-1} \Gamma_{\alpha \gamma}^{k} \theta_{1} \ldots \theta_{\gamma-1} \widetilde{Q}\left(\eta_{\alpha \gamma}\right) \theta_{\gamma+1} \ldots \theta_{16} G\right)+ \\
& +\operatorname{tr}\left(\sum_{\gamma=1}^{\alpha-1}(-1)^{\gamma-1} \Gamma_{\alpha \gamma}^{k} \theta_{1} \ldots \theta_{\gamma-1} D_{k} \theta_{\gamma+1} \ldots \theta_{16} G\right)+ \\
& +\frac{1}{2} \operatorname{tr}\left((-1)^{\alpha-1} \Gamma_{\alpha \alpha}^{k} \theta_{1} \ldots \theta_{\alpha-1} \widetilde{Q}\left(\eta_{\alpha \gamma}\right) \theta_{\alpha+1} \ldots \theta_{16} G\right)+ \\
& +\frac{1}{2} \operatorname{tr}\left((-1)^{\alpha-1} \Gamma_{\alpha \alpha}^{k} \theta_{1} \ldots \theta_{\alpha-1} D_{k} \theta_{\alpha+1} \ldots \theta_{16} G\right)= \\
& =Q \sum_{\gamma=1}^{\alpha-1} \Gamma_{\alpha \gamma}^{k} \operatorname{tr}\left(\theta_{1} \ldots \theta_{\gamma-1} \eta_{\alpha \gamma} \theta_{\gamma+1} \ldots \theta_{16} G\right)+ \\
& +Q \frac{1}{2} \Gamma_{\alpha \alpha}^{k} \operatorname{tr}\left(\theta_{1} \ldots \theta_{\alpha-1} \eta_{\alpha \alpha} \theta_{\alpha+1} \ldots \theta_{16} G\right)+ \\
& +Q \sum_{\gamma=1}^{\alpha-1} \sum_{\gamma^{\prime}=1}^{\gamma-1}(-1)^{\gamma+\gamma^{\prime}} \Gamma_{\alpha \gamma}^{k} \operatorname{tr}\left(\theta_{1} \ldots \theta_{\gamma^{\prime}-1} \eta_{\alpha k} \theta_{\gamma^{\prime}+1} \ldots \hat{\theta}_{\gamma} \ldots \theta_{16} G\right)+ \\
& +Q \frac{1}{2} \sum_{\gamma=1}^{\alpha-1}(-1)^{\alpha+\gamma} \Gamma_{\alpha \alpha}^{k} \operatorname{tr}\left(\theta_{1} \ldots \theta_{\gamma-1} \eta_{\alpha k} \theta_{\gamma+1} \ldots \hat{\theta}_{\alpha} \ldots \theta_{16} G\right)+ \\
& +\sum_{\gamma=1}^{\alpha-1} \frac{\partial}{\partial x^{k}} \operatorname{tr}\left((-1)^{\gamma-1} \Gamma_{\alpha \gamma}^{k} \theta_{1} \ldots \hat{\theta}_{\gamma} \ldots \theta_{16} G\right)+ \\
& +\frac{1}{2} \frac{\partial}{\partial x^{k}} \operatorname{tr}\left((-1)^{\alpha-1} \Gamma_{\alpha \alpha}^{k} \theta_{1} \ldots \hat{\theta}_{\alpha} \ldots \theta_{16} G\right) .
\end{aligned}
$$

As usual " " marks the symbol that should be omitted in the formula. If subscript in $\theta_{\gamma}$ is out of range $[1,16]$ then $\theta_{\gamma}$ must be omitted. From this computation we conclude that for Hamiltonian of the vector field $q_{\alpha}$ as a function of $G$ is

$$
\begin{aligned}
& \sum_{\gamma=1}^{\alpha-1} \Gamma_{\alpha \gamma}^{k} \operatorname{tr}\left(\theta_{1} \ldots \theta_{\gamma-1} \eta_{\alpha \gamma} \theta_{\gamma+1} \ldots \theta_{16} G\right)+ \\
& +\frac{1}{2} \Gamma_{\alpha \alpha}^{k} \operatorname{tr}\left(\theta_{1} \ldots \theta_{\alpha-1} \eta_{\alpha \alpha} \theta_{\alpha+1} \ldots \theta_{16} G\right)+ \\
& +\sum_{\gamma=1}^{\alpha-1} \sum_{\gamma^{\prime}=1}^{\gamma-1}(-1)^{\gamma+\gamma^{\prime}} \Gamma_{\alpha \gamma}^{k} \operatorname{tr}\left(\theta_{1} \ldots \theta_{\gamma^{\prime}-1} \eta_{\alpha k} \theta_{\gamma^{\prime}+1} \ldots \hat{\theta}_{\gamma} \ldots \theta_{16} G\right)+ \\
& +\frac{1}{2} \sum_{\gamma=1}^{\alpha-1}(-1)^{\alpha} \Gamma_{\alpha \alpha}^{k} \operatorname{tr}\left(\theta_{1} \ldots \theta_{\gamma-1} \eta_{\alpha k} \theta_{\gamma+1} \ldots \hat{\theta}_{\alpha} \ldots \theta_{16} G\right)
\end{aligned}
$$




\section{Formal SUSY deformations}

We have analyzed infinitesimal SUSY deformations of reduced and unreduced SUSY YM theory. One can prove that all of these deformations can be extended to formal deformations (i.e. there exist SUSY deformations represented as formal series with respect to parameter $\epsilon$ and giving an arbitrary infinitesimal deformation in the first order with respect to $\epsilon$ ). We shall sketch the proof of this fact in present section.

We have seen in Section [6 that there is a large class of infinitesimal supersymmetric deformations that have a form $\theta_{1} \ldots \theta_{16} G$. We shall start with the proof that all these infinitesimal deformations can be extended to formal deformations.

We shall consider more general situation when we have any action functional in $\mathrm{BV}$ formalism that is invariant with respect to $\mathrm{L}_{\infty}$ action of SUSY. As follows from Appendix D our considerations can be applied to ten- dimensional SYM theory.

The SUSY Lie algebra has $m$ even commuting generators $X_{1}, \ldots, X_{m}$ and $n$ odd generators $\tau_{1}, \ldots, \tau_{n}$ obeying relations

$$
\left[\tau_{\alpha}, \tau_{\beta}\right]=\Gamma_{\alpha \beta}^{i} X_{i}
$$

In the definition of $\mathrm{L}_{\infty}$-action of $\mathfrak{g}$ we use the algebra $C^{\bullet}(\mathfrak{g})$ of functions of corresponding ghosts. In our case this algebra is the algebra

$$
K=\mathbb{C}\left[\left[t^{1}, \ldots, t^{n}\right]\right] \otimes \Lambda\left[\xi^{1}, \ldots, \xi^{m}\right] .
$$

The odd variables $\xi^{1}, \ldots, \xi^{m}$ are the ghosts for even generators (space-time translations), the even variables are the ghosts for odd generators. The algebra $K$ is equipped with the differential

$$
d=\Gamma_{\alpha \beta}^{i} t^{\alpha} t^{\beta} \frac{\partial}{\partial \xi_{i}}
$$

where $\Gamma_{\alpha \beta}^{i}$ are the structure constants of the supersymmetry algebra. 
The $\mathrm{L}_{\infty}$ action can be described by an element $\hat{S} \in A=K \otimes C^{\infty}(M)$, where $M$ is the space of fields (in other words $\hat{S}$ is a function of ghost variables $t^{i}, \xi^{\alpha}$ and fields).

The equation (93) for $\hat{S}$ takes the form

$$
d \hat{S}+\frac{1}{2}\{\hat{S}, \hat{S}\}=0 .
$$

A solution to this equation gives us a solution $S$ to the BV Master equation (obtained if we assume that ghost variables are equal to zero) and $\mathrm{L}_{\infty}$ action of supersymmetries preserving $S$. We would like to construct a formal deformation of such a solution, i.e. we would like to construct a formal power series $\hat{S}(\epsilon)$ with respect to $\epsilon$ obeying the equation (106) and giving the original solution for $\epsilon=0$. We shall start with a construction of the solution of the equation for infinitesimal deformation

$$
d H+\{\hat{S}, H\}=0
$$

If we know the solution of the equation (107) for every $\hat{S}$ we can find the deformation solving the equation

$$
\frac{d \hat{S}(\epsilon)}{d \epsilon}=H(\hat{S}(\epsilon))
$$

To solve the equation (107) we construct a family of functions $F^{k}$ defined by inductive formula

$$
F^{k+1}=\frac{1}{t_{k+1}}\left(d_{k+1} F^{k}+\left\{\hat{S}^{k}, F^{k}\right\}\right) .
$$

where $d_{k}$ is defined as $\sum_{\alpha \beta \leq k} \Gamma_{\alpha \beta}^{i} t^{\alpha} t^{\beta} \frac{\partial}{\partial \xi_{i}}$. We assume that $F^{k}$ and $\hat{S}^{k}$ do not depend on $t^{k+1}, \ldots, t^{n}$ and $\hat{S}^{k}$ coincides with $\hat{S}$ if $t^{k+1}=\cdots=t^{n}=0$. We impose also an initial condition $F^{0}$ obeying $\left\{\hat{S}_{i}, F^{0}\right\}=0$ where $\hat{S}_{i}=\left.\frac{\partial \hat{S}}{\partial \xi_{i}}\right|_{0}$. We shall see that $F^{n}$ is a solution of the equation (107); this allows us to take $H=F^{n}$. To prove this fact we should give geometric interpretation of (109). First of all we notice that the solutions of (107) are cocycles of the differential $d_{S}=d+\{\hat{S}, \cdot\}$ acting on the algebra $A$ of functions of ghosts $t^{1}, \ldots, t^{n}, \xi^{1}, \ldots, \xi^{m}$ and fields. We consider differential ideal $I_{k}$ of this algebra defined as set of 
functions that vanish if $t^{1}=\cdots=t^{k}=0$ (in other words, $I_{k}$ is generated by $\left.t^{1}, \ldots, t^{k}\right)$ and the quotient $A_{k}$ of the algebra $A$ with respect to this ideal. The differential algebra $A_{k}$ can be interpreted as the algebra of functions depending on ghosts $t^{1}, \ldots, t^{k}, \xi^{1}, \ldots, \xi^{m}$ and fields. The inductive formula (109) gives a map of $A_{k}$ into $A_{k+1}$ that descends to cohomology. To construct this map we notice that the embedding $I_{k+1} \subset I_{k}$ generates a short exact sequence

$$
0 \rightarrow I_{k} / I_{k+1} \rightarrow A_{k+1} \rightarrow A_{k} \rightarrow 0
$$

The ideal $I_{k} / I_{k+1}$ of the algebra $A_{k+1}$ is generated by one element $t^{k+1}$. This means we can rewrite the exact sequence in the form

$$
0 \rightarrow A_{k+1} \rightarrow A_{k+1} \rightarrow A_{k} \rightarrow 0
$$

where the map $A_{k+1} \rightarrow A_{k+1}$ is a multiplication by $t^{k+1}$. The boundary map in the corresponding exact cohomology sequence gives (109). The condition imposed on $F^{0}$ means that $F^{0}$ is a cocycle in $A_{0}$.

For every admissible $F^{0}$ we have constructed $H(S)$ as a solution of (107); we have used this solution to construct formal deformation by means of (108).

This fairly simple description of supersymmetric deformations has one obvious shortcoming. The Poincaré invariance is hopelessly lost in the the formula (109) even if we start with Poincaré invariant $F^{0}$. This can be fixed if we work in the euclidean signature. The algebra $A$ contains a subalgebra $A_{\mathrm{SO}(m)}$ of $\mathrm{SO}(m)$-invariant elements. The vector field $H\left(F^{0}\right)$, restricted on $A_{\mathrm{SO}(m)}$ can be replaced by

$$
H^{\mathrm{SO}(m)}=\frac{1}{\operatorname{vol}(\mathrm{SO}(m))} \int_{\mathrm{SO}(m)} H^{g} d g
$$

- the average of the $g$-rotated element $H$ over $\mathrm{SO}(m)$. It can be proved by other means that $H_{F^{0}}^{\mathrm{SO}(m)}$ is nonzero if $F^{0}$ is Poincaré-invariant. The above prescription can be formulated also in more algebraic form where Euclidean signature is unnecessary. We decompose $A$ into direct sum of irreducible representations of $\mathrm{SO}(m)$ and leave only $\mathrm{SO}(m)$ invariant part of $H$.

Let us make a connection with Section 3 . 
We start with identifications. The odd symplectic manifold $M$ coincides with the space of fields in the maximally super- symmetric Yang-Mills theory in Batalin-Vilkovisky formalism (we can consider both reduced case when $n=$ $16, m=0$ and unreduced case when $n=16, m=10$ ). It can be shown that the supersymmetry action can be extended to an $\mathrm{L}_{\infty}$ action, whose generating function satisfies equation (106); see Appendix D,

Let us start with a Poincaré invariant $F^{0}=G$ as described in Section 3. The l'Hôpital's rule applied to $H=F^{n}$ shows that its leading term coincides with (56). This means that infinitesimal SUSY deformations of the form $\operatorname{tr} \theta_{1} \cdots \theta_{16} G$ can be extended to formal deformations. In reduced case this logic can be applied to arbitrary Poincaré invariant $G$, in unreduced case we should consider local gauge covariant $G$ to obtain SUSY deformation.

There exists only one infinitesimal deformation $\delta \mathcal{L}_{16}$ that does not have the form $\operatorname{tr} \theta_{1} \cdots \theta_{16} G$ (Theorem 9 and Theorem 10). 20 One can prove that this deformation also can be extended to formal deformation together with $\mathrm{L}_{\infty}$ action of SUSY algebra (7) 21 Constructing formal deformations of this formal deformation we obtain that in the reduced case all infinitesimal deformations can be extended to formal ones.

We have noticed in Section 3 that for $G$ of the form $\Delta=\nabla_{i} \nabla_{i}$ the expression $\operatorname{tr} \theta_{1} \cdots \theta_{16} G$ generates a SUSY infinitesimal deformation of unreduced YM action functional. One can prove that this deformation also can be extended to formal deformation, however, the above construction of formal deformation does not work in this case. The proof is based on the remark that infinitesimal deformation $A \operatorname{tr} \Delta$ can be applied to a formal deformation we constructed and it remains local.

\footnotetext{
${ }^{20}$ It is better to say that every infinitesimal deformation can be represented as linear combination of $\delta \mathcal{L}_{16}$ and $\operatorname{tr} \theta_{1} \cdots \theta_{16} G$.

${ }^{21}$ Notice that superstring theory gives a formal SUSY deformation of SYM theory that corresponds to infinitesimal deformation represented as linear combination of $\delta \mathcal{L}_{16}$ (with nonzero coefficient) and $\operatorname{tr} \theta_{1} \cdots \theta_{16} G$. If we were able to prove that that SUSY action extends to $\mathrm{L}_{\infty}$ action we could use this deformation to extend all infinitesimal deformations in reduced case.
} 


\section{Appendices}

\section{A $\quad \mathbf{L}_{\infty}$ and $\mathbf{A}_{\infty}$ algebras}

Let us consider a supermanifold equipped with an odd vector field $Q$ obeying $[Q, Q]=0$ (a $Q$-manifold). Let us introduce a coordinate system in a neighborhood of a point of $Q$-manifold belonging to zero locus $Q$. Then the vector field $Q$ considered as a derivation of the algebra of formal power series can be specified by its action on the coordinate functions $z^{A}$ :

$$
Q\left(z^{A}\right)=\sum_{n} \sum \pm \mu_{B_{1}, \ldots, B_{n}}^{A} z^{B_{1}} \ldots z^{B_{n}}
$$

We can use tensors $\mu_{n}=\mu_{B_{1}, \ldots, B_{n}}^{A}$ to define a series of operations. The operation $\mu_{n}$ has $n$ arguments; it can be considered as a linear map $V^{\otimes n} \rightarrow V$ (here $V$ stands for the tangent space at $x=0$ ). However, it is convenient to change parity of $V$ and consider $\mu_{n}$ as a symmetric map $(\Pi V)^{\otimes n} \rightarrow \Pi V$. It is convenient to add some signs in the definition of $\mu_{n}$. With appropriate choice of signs we obtain that operations $\mu_{n}$ obey some quadratic relations; by definition the operators $\mu_{n}$ obeying these relations specify a structure of $\mathrm{L}_{\infty}$ algebra on $W=\Pi V$. We see that a point of zero locus of the field $Q$ specifies an $\mathrm{L}_{\infty}$ algebra; geometrically one can say that $\mathrm{L}_{\infty}$ algebra is a formal $Q$-manifold. (A formal manifold is a space whose algebra of functions can be identified with the algebra of formal power series. If the algebra is equipped with odd derivation $Q$, such that $\{Q, Q\}=0$ we have a structure of formal $Q$ manifold.) The considerations of our paper are formal. This means that we can interpret all functions of fields at hand as formal power series. Therefore instead of working with $Q$-manifolds we can work with $\mathrm{L}_{\infty}$ algebras.

On a $Q$-manifold with odd symplectic structure we can choose the coordinates $z^{1}, \ldots, z^{n}$ as Darboux coordinates,i.e. we can assume that the coefficients of symplectic form do not depend on $z$. Then the $\mathrm{L}_{\infty}$ algebra is equipped with invariant odd inner product. 
Hence we can say that $\mathrm{L}_{\infty}$ algebra specifies a classical system and $\mathrm{L}_{\infty}$ algebra with invariant odd inner product specifies a Lagrangian classical system.

It is often important to consider $\mathbb{Z}$-graded $\mathrm{L}_{\infty}$-algebras (in $\mathrm{BV}$-formalism this corresponds to the case when the fields are classified according to ghost number). We assume in this case that the derivation $Q$ raises the grading (the ghost number) by one.

An $\mathrm{L}_{\infty}$ algebra where all operations $\mu_{n}$ with $n \geq 3$ vanish can be identified with differential graded Lie algebra (the operation $\mu_{1}$ is the differential, $\mu_{2}$ is the bracket). An $\mathrm{L}_{\infty}$ algebra corresponding to Lie algebra with zero differential is $\mathbb{Z}$-graded.

For $\mathrm{L}_{\infty}$ algebra $\mathfrak{g}=\left(W, \mu_{n}\right)$ one can define a notion of cohomology generalizing the standard notion of cohomology of Lie algebra. For example, in the case of trivial coefficients we can consider cohomology of $Q$ acting as a derivation of the algebra $\widehat{\operatorname{Sym}}\left(W^{*}\right)$ of formal functions on $W$ (of the algebra of formal series). In the case when the $\mathrm{L}_{\infty}$ algebra corresponds to differential Lie algebra $\mathfrak{g}$ this cohomology coincides with Lie algebra cohomology $\mathrm{H}(\mathfrak{g}, \mathbb{C})$ (cohomology with trivial coefficients). Considering cohomology of $Q$ acting on the space of vector fields (space of derivations of the algebra of functions) we get a notion generalizing the notion of cohomology $\mathrm{H}(\mathfrak{g}, \mathfrak{g})$ ( cohomology with coefficients in adjoint representation).

Notice, that to every $\mathrm{L}_{\infty}$ algebra $\mathfrak{g}=\left(W, \mu_{n}\right)$ we can assign a supercommutative differential algebra $\left(\widehat{\operatorname{Sym}}\left(W^{*}\right), Q\right)$ that is in some sense dual to the original $\mathrm{L}_{\infty}$-algebra. If only a finite number of operations $\mu_{n}$ does not vanish the operator $Q$ transforms a polynomial function into a polynomial function, hence we can consider also a free supercommutative differential algebra $(\operatorname{Sym}(W), Q)$ where $\operatorname{Sym}(W)$ stands for the algebra of polynomials on $W$. We shall use the notations $\left(\operatorname{Sym}\left(W^{*}\right), Q\right)=C^{\bullet}(\mathfrak{g}),\left(\widehat{\operatorname{Sym}}\left(W^{*}\right), Q\right)=\hat{C}^{\bullet}(\mathfrak{g})$ and the notations $\mathrm{H}(\mathfrak{g}, \mathbb{C}), \hat{\mathrm{H}}(\mathfrak{g}, \mathbb{C})$ for corresponding cohomology. 23 Similarly for the cohomology

\footnotetext{
22 Usually the definition of Lie algebra cohomology is based on the consideration of polynomial functions of ghosts; using formal series we obtain a completion of cohomology.

${ }^{23}$ In the case of Lie algebra the functor $C^{\bullet}$ coincides with Cartan-Eilenberg construction of
} 
in the space of derivations we use the notations $\mathrm{H}(\mathfrak{g}, \mathfrak{g}), \hat{\mathrm{H}}(\mathfrak{g}, \mathfrak{g})$.

In the case when $\mathrm{L}_{\infty}$ algebra is $\mathbb{Z}$-graded the cohomology $\mathrm{H}(\mathfrak{g}, \mathbb{C})$ and $\mathrm{H}(\mathfrak{g}, \mathfrak{g})$ are also $\mathbb{Z}$-graded.

One can consider intrinsic cohomology of an $\mathrm{L}_{\infty}$ algebra. They are defined as $\operatorname{Ker} \mu_{1} / \operatorname{Im} \mu_{1}$. One says that an $\mathrm{L}_{\infty}$ homomorphism, which is the same as $Q$-map in the language of $Q$-manifold 24 , is a quasi-isomorphism if it induces an isomorphism of intrinsic cohomology. Notice, that in the case of $\mathbb{Z}$-graded $\mathrm{L}_{\infty}$ algebras $\mathrm{L}_{\infty}$ homomorphism should respect $\mathbb{Z}$ grading.

Every $\mathbb{Z}$-graded $\mathrm{L}_{\infty}$ algebra is quasi-isomorphic to $\mathrm{L}_{\infty}$ with $\mu_{1}=0$. (In other words every $\mathrm{L}_{\infty}$ algebra has a minimal model). Moreover, every $\mathbb{Z}$-graded $\mathrm{L}_{\infty}$ algebra algebra is quasi-isomorphic to direct product of minimal $\mathrm{L}_{\infty}$ algebra and a trivial one. (We say that $\mathrm{L}_{\infty}$ algebra is trivial if it can be regarded as differential abelian Lie algebra with zero cohomology.)

The role of zero locus of $Q$ is played by the space of solutions of MaurerCartan (MC) equation:

$$
\sum_{n} \frac{1}{n !} \mu_{n}(a, \ldots, a)=0 .
$$

To obtain a space of solutions $\mathrm{Sol} / \sim$ we should factorize space of solutions $\mathrm{Sol}$ of MC in appropriate way or work with a minimal model of $A$.

Our main interest lies in gauge theories. We consider these theories for all groups $\mathrm{U}(n)$ at the same time. To analyze these theories it is more convenient to work with $\mathrm{A}_{\infty}$ instead of $\mathrm{L}_{\infty}$ algebras.

An $\mathrm{A}_{\infty}$ algebra can be defined as a formal non-commutative $Q$-manifold. In other words we consider an algebra of power series of several variables which do not satisfy any relations (some of them are even, some are odd). An $\mathrm{A}_{\infty}$ algebra is defined as an odd derivation $Q$ of this algebra which satisfies $[Q, Q]=0$.

More precisely we consider a $\mathbb{Z}_{2}$-graded vector space $W$ with coordinates $z^{A}$. The algebra of formal noncommutative power series $\mathbb{C}\left\langle\left\langle z^{A}\right\rangle\right\rangle$ is a completion $\hat{T}\left(W^{*}\right)$ of the tensor algebra $T\left(W^{*}\right)$ (of the algebra of formal noncommutative

differential algebra giving Lie algebra cohomology.

${ }^{24}$ Recall, that a map of $Q$-manifolds is a $Q$-map if it is compatible with $Q$. 
polynomials). The derivation is specified by the action on $z^{A}$ :

$$
Q\left(z^{A}\right)=\sum_{n} \sum \pm \mu_{B_{1}, \ldots, B_{n}}^{A} z^{B_{1}} \ldots z^{B_{n}}
$$

We can use $\mu_{B_{1}, \ldots, B_{n}}^{A}$ to specify a series of operations $\mu_{n}$ on the space $\Pi W$ as in $\mathrm{L}_{\infty}$ case. (In the case when $W$ is $\mathbb{Z}$-graded instead parity reversal $\Pi$ we should consider the shift of the grading by 1.) If $Q$ defines an $\mathrm{A}_{\infty}$ algebra then the condition $[Q, Q]=0$ leads to quadratic relations between operations; these relations can be used to give an alternative definition of $\mathrm{A}_{\infty}$ algebra. In this definition an $\mathrm{A}_{\infty}$ algebra is a $\mathbb{Z}_{2}$-graded or $\mathbb{Z}$ - graded linear space, equipped with a series of maps $\mu_{n}: A^{\otimes n} \rightarrow A, n \geq 1$ of degree $2-n$ that satisfy quadratic relations:

$$
\begin{aligned}
& \sum_{i+j=n+1} \sum_{0 \leq l \leq i} \epsilon(l, j) \times \\
& \mu_{i}\left(a_{0}, \ldots, a_{l-1}, \mu_{j}\left(a_{l}, \ldots, a_{l+j-1}\right), a_{l+j}, \ldots, a_{n}\right)=0
\end{aligned}
$$

where $a_{m} \in A$, and

$$
\epsilon(l, j)=(-1)^{j \sum_{0 \leq s \leq l-1} \operatorname{deg}\left(a_{s}\right)+l(j-1)+j(i-1)} .
$$

In particular, $\mu_{1}^{2}=0$.

Notice that in the case when only finite number of operations $\mu_{n}$ do not vanish (the RHS of (111) is a polynomial) we can work with polynomial functions instead of power series. We obtain in this case a differential on the tensor algebra $\left(T\left(\Pi W^{*}\right), Q\right)$. The transition from $\mathrm{A}_{\infty}$ algebra $A=\left(W, \mu_{n}\right)$ to a differential algebra cobar $A=\left(T\left(\Pi W^{*}\right), Q\right)$ is known as co- bar construction. If we consider instead of tensor algebra its completion (the algebra of formal power series) we obtain the differential algebra $\left(\hat{T}\left(\Pi W^{*}\right), Q\right)$ as a completed co-bar construction $\widehat{\operatorname{cobar}} A$.

The cohomology of differential algebra $\left(T\left(\Pi W^{*}\right), Q\right)=\operatorname{cobar} A$ are called Hochschild cohomology of $A$ with coefficients in trivial module $\mathbb{C}$; they are denoted by $\mathrm{HH}(A, \mathbb{C})$. Using the completed co-bar construction we can give another definition of Hochschild cohomology of $\mathrm{A}_{\infty}$ algebra as the cohomology of the differential algebra $\left(\hat{T}\left(\Pi W^{*}\right), Q\right)=\widehat{\operatorname{cobar}} A$; this cohomology can be defined also in 
the case when we have infinite number of operations. It will be denoted by $\widehat{\mathrm{HH}}(A, \mathbb{C})$. Under some mild conditions (for example, if the differential is equal to zero) one can prove that $\widehat{\mathrm{HH}}(A, \mathbb{C})$ is a completion of $\mathrm{HH}(A, \mathbb{C})$; in the case when $\operatorname{HH}(A, \mathbb{C})$ is finite-dimensional this means that the definitions coincide. We shall always assume that $\widehat{\mathrm{HH}}(A, \mathbb{C})$ is a completion of $\mathrm{HH}(A, \mathbb{C})$.

The theory of $\mathrm{A}_{\infty}$ algebras is very similar to the theory of $\mathrm{L}_{\infty}$ algebras. In particular $\mu_{1}$ is a differential: $\mu_{1}^{2}=0$. It can be used to define intrinsic cohomology of $\mathrm{A}_{\infty}$ algebra. If $\mu_{n}=0$ for $n \geq 3$ then operations $\mu_{1}, \mu_{2}$ define a structure of differential associative algebra on $W$.

The role of equations of motion is played by so called $\mathrm{MC}$ equation

$$
\sum_{n \geq 1} \mu_{n}(a, \ldots, a)=0
$$

Again to get a space of solutions $\mathrm{Sol} / \sim$ we should factorize solutions of MC equation in appropriate way or to work in a framework of minimal models, i.e. we should use the $A_{\infty}$ algebra that is quasi-isomorphic to the original algebra and has $\mu_{1}=0$. (Every $\mathbb{Z}$-graded $\mathrm{A}_{\infty}$ algebra has a minimal model.)

We say that 1 is the unit element of $\mathrm{A}_{\infty}$ algebra if $\mu_{2}(1, a)=\mu_{2}(a, 1)=a$ (i.e. 1 is the unit for binary operation) and all other operations with 1 as one of arguments give zero. For every $\mathrm{A}_{\infty}$ algebra $A$ we construct a new $\mathrm{A}_{\infty}$ algebra $\tilde{A}$ adjoining a unit element. 25

Having an $\mathrm{A}_{\infty}$ algebra $A$ we can construct a series $L_{N}(A)$ of $\mathrm{L}_{\infty}$ algebras. If $N=1$ it is easy to describe the corresponding $\mathrm{L}_{\infty}$ algebra in geometric language. There is a map from noncommutative formal functions on $\Pi A$ to ordinary (super)commutative formal functions on the same space. Algebraically it corresponds to imposing (super) commutativity relations among generators. Derivation $Q$ is compatible with such modification. It results in $L_{1}(A)$. By definition $L_{N}(A)=L_{1}\left(A \otimes \operatorname{Mat}_{N}\right)$.

\footnotetext{
25 Notice, that in our definition of Hochschild cohomology we should work with non-unital algebras; otherwise the result for the cohomology with coefficients in $\mathbb{C}$ would be trivial. In more standard approach one defines Hochschild cohomology of unital algebra using the augmentation ideal.
} 
If $A$ is an ordinary associative algebra, then $L_{1}(A)$ is in fact a Lie algebra- it has the same space and the operation is equal to the commutator $[a, b]=a b-b a$.

The use of $\mathrm{A}_{\infty}$ algebras in the YM theory is based on the remark that one can construct an $\mathrm{A}_{\infty}$ algebra $\mathcal{A}$ with inner product such that for every $N$ the algebra $L_{N}(\tilde{\mathcal{A}})$ specifies $\mathrm{YM}$ theory with matrices of size $N \times N$ in $B V$ formalism. (Recall, that we construct $\tilde{\mathcal{A}}$ adjoining unit element to $\mathcal{A}$.) The construction of the $\mathrm{A}_{\infty}$ algebra $\tilde{\mathcal{A}}$ is very simple: in the formula for $Q$ in BV-formalism of YM theory in component formalism we replace matrices with free variables. The operator $Q$ obtained in this way specifies also differential algebras cobar $\tilde{\mathcal{A}}$ and $\widehat{\operatorname{cobar}} \tilde{\mathcal{A}}$. To construct the $\mathrm{A}_{\infty}$ algebra $\mathcal{A}$ in the case of reduced YM theory we notice that the elements of the basis of $\tilde{\mathcal{A}}$ correspond to the fields of the theory; the element corresponding to the ghost field $c$ is the unit; remaining elements of the basis generate the algebra $\mathcal{A}$. In the case of reduced theory the differential algebra $\operatorname{cobar} \mathcal{A}$ can be obtained from $\operatorname{cobar} \tilde{\mathcal{A}}$ by means of factorization with respect to the ghost field $c$; we denote this algebra by $B V_{0}$ and the original algebra $\mathcal{A}$ will be denoted by $b v_{0}$. The construction in unreduced case is similar. In this case the ghost field (as all other fields) is a function on ten-dimensional space; to obtain $\operatorname{cobar} \mathcal{A}$ (that will be denoted later by $B V$ ) we factorize cobar $\tilde{\mathcal{A}}$ with respect to the ideal generated by the constant ghost field $c$. We shall use the notation $b v$ for the algebra $\mathcal{A}$ in unreduced case.

Instead of working with component fields we can use pure spinors. Then instead of the algebra $b v_{0}$ we should work with reduced Berkovits algebra $B_{0}$ that is quasi-isomorphic to $b v_{0}$; the algebra $B V_{0}$ is quasi-isomorphic to $U(Y M)$. In unreduced case we work with Berkovits algebra $B$ that is quasi-isomorphic to $b v$ and with the algebra $U(T Y M)$ quasi-isomorphic to $B V$ (see Section 6 , [34] and 35] for more detail).

Notice, that the quasi-isomorphisms we have described are useful for calculation of homology. For example, as we have seen in Section [ the space of fields in pure spinor formalism can be equipped with odd symplectic form (100) that vanishes if the sum of ghost numbers of arguments is $>3$; the space of fields should be factorized with respect to the kernel of this form. It follows that 
homology and cohomology of $U(Y M)$ with coefficients in any module vanish in dimensions $>3$. From the other side the form (100) can be used to establish Poincaré duality in the cohomology of $U(Y M)$.

It is easy to reduce classification of deformations of $\mathrm{A}_{\infty}$ algebra $A$ to a homological problem (see [38]). Namely it is clear that an infinitesimal deformation of $Q$ obeying $[Q, Q]=0$ is an odd derivation $q$ obeying $[Q, q]=0$. The operator $Q$ specifies a differential on the space of all derivations by the formula

$$
\widetilde{Q} q=[Q, q]
$$

We see that infinitesimal deformations correspond to cocycles of this differential. It is easy to see that two infinitesimal deformations belonging to the same cohomology class are equivalent (if $q=[Q, v]$ where $v$ is a derivation then we can eliminate $q$ by a change of variables $\exp (v)$ ). We see that the classes of infinitesimal deformations can be identified with homology $\mathrm{H}(V e c t(\mathbb{V}), d)$ of the space of vector fields. (Vector fields on $\mathbb{V}$ are even and odd derivations of $\mathbb{Z}_{2^{-}}$ graded algebra of formal power series.) If the number of operations is finite we can restrict ourselves to polynomial vector fields (in other words, we can replace $V e c t(\mathbb{V})$ with $\operatorname{cobar} A \otimes A)$.

The above construction is another particular case of Hochschild cohomology ( the cohomology with coefficients in coefficients in $\mathbb{C}$ was defined in terms of cobar construction. ) We denote it by $\widehat{\mathrm{HH}}(A, A)$ (if we are working with formal power series) or by $\operatorname{HH}(A, A)$ (if we are working with polynomials). Notice that these cohomologies have a structure of (super) Lie algebra induced by commutator of vector fields.

We shall give a definition of Hochschild cohomology of differential graded associative algebra $\left(A, d_{A}\right)$

$$
A=\bigoplus_{i \geq 0} A_{i}
$$

with coefficients in a differential bimodule $\left(M, d_{M}\right)$

$$
M=\bigoplus_{i} M_{i}
$$


in terms of Hochschild cochains (multilinear functionals on $A$ with values in $M$ ).

We use the standard notation for the degree $\bar{a}=i$ of a homogeneous element $a \in A_{i}$.

We first associate with the pair $(A, M)$ a bicomplex $\left(C^{n, m}, D_{I}, D_{I I}\right), n \geq 0$, $D_{I}: C^{n, m} \rightarrow C^{n+1, m}, D_{I I}: C^{n, m} \rightarrow C^{n, m+1}$ as follows:

$$
C^{n . m}(A, M)=\prod_{i_{1}, \ldots, i_{n}} \operatorname{Hom}\left(A_{i_{1}} \otimes \cdots \otimes A_{i_{n}}, M_{m+i_{1}+\cdots i_{n}}\right)
$$

and for $c \in C^{n, m}$

$$
\begin{aligned}
& D_{I} c=a_{0} c\left(a_{1}, \ldots, a_{n}\right)+\sum_{i=0}^{n-1}(-1)^{i+1} c\left(a_{0}, \ldots, a_{i} a_{i+1}, \ldots, a_{n}\right)+(-1)^{m \bar{a}_{n}+n} c\left(a_{0}, \ldots, a_{n-1}\right) a_{n} \\
& D_{I I} c=\sum_{i=1}^{n}(-1)^{1+\bar{a}_{1}+\cdots a_{i-1}} c\left(a_{1}, \ldots, d_{A}\left(a_{i}\right), \ldots, a_{n}\right)+(-1)^{k} d_{M} c\left(a_{1}, \ldots, a_{n}\right)
\end{aligned}
$$

Clearly

$$
D_{I}^{2}=0, D_{I I}^{2}=0, D_{I} D_{I I}+D_{I I} D_{I}=0
$$

We define the space of Hochschild $i$-th cochains as

$$
\widehat{C}^{i}(A, M)=\prod_{n+m=i} C^{n, m}(A, M)^{\natural} .
$$

Then $\widehat{C}^{\bullet}(A, M)$ is the complex $\left(\prod C^{i}(A, M), D\right)$ with $D=D_{I}+D_{I I}$. The operator $D$ can also be considered as a differential on the direct sum $C(A, M)=$ $\bigoplus_{i} C^{i}(A, M)$ with direct products in (115[117) replaced by the direct sums (on the space of non-commutative polynomials on $\Pi A$ with values in $M$ ). Similarly $\widehat{C}(A, M)$ gets interpreted as the space of formal power series on $A$ with values in $M$. We define the Hochschild cohomology $\operatorname{HH}(A, M)$ and $\widehat{\mathrm{HH}}(A, M)$ as the cohomology of this differential. Again under certain mild conditions that will be assumed in our consideration the second group is a completion of the first one; the groups coincide if $\operatorname{HH}(A, M)$ is finite-dimensional.

Notice that $C(A, M)$ can be identified with the tensor product $\operatorname{cobar} A \otimes M$ with a differential defined by the formula

$$
D(c \otimes m)=\left(d_{\text {cobar }}+d_{M}\right) c \otimes m+[e, c \otimes m]
$$


where $e$ is the tensor of the identity map id $\in \operatorname{End}(A) \cong \Pi A^{*} \otimes A \subset \operatorname{cobar}(A) \otimes A$

A similar statement is true for $\hat{C}(A, M)$.

Notice that we can define the total grading of Hochschild cohomology $\operatorname{HH}^{i}(A, M)$ where $i$ stands for the total grading defined in terms of $A, M$ and the ghost number (the number of arguments).

In the case when $M$ is the algebra $A$ considered as a bimodule the elements of $\operatorname{HH}^{2}(A, A)$ label infinitesimal deformations of associative algebra $A$ and the elements of $\mathrm{HH}^{\bullet}(A, A)$ label infinitesimal deformations of $A$ into $\mathrm{A}_{\infty}$ algebra. Derivations of $A$ specify elements of $\operatorname{HH}^{1}(A, A)$ (more precisely, a derivation can be considered as one-dimensional Hochschild cocycle; inner derivations are homologous to zero).

We can define Hochschild homology HH• considering Hochschild chains (elements of $A \otimes \ldots \otimes A \otimes M$ ). If $A$ and $M$ are finite-dimensional (or graded with finite-dimensional components) we can define homology by means of dualization of cohomology

$$
\mathrm{HH}_{i}(A, M)=\mathrm{HH}^{i}\left(A, M^{*}\right)^{*}
$$

Let us assume that the differential bimodule $M$ is equipped with bilinear inner product of degree $n 26$ that descends to non-degenerate inner product on homology. This product generates a quasi-isomorphism $M \rightarrow M^{*}$ and therefore an isomorphism between $\mathrm{HH}_{i}(A, M)$ and $\mathrm{HH}^{n-i}(A, M)$ (Poincaré isomorphism). Let us suppose now that $A_{\infty}$ has Lie algebra of symmetries $\mathfrak{g}$ and we are interested in deformations of this algebra preserving the symmetries.

This problem appears if we consider YM theory for all groups $\mathrm{U}(n)$ at the same time and we would like to deform the equations of motion preserving the symmetries of the original theory (however we do not require that the deformed equations come from an action functional).

When we are talking about symmetries of $\mathrm{A}_{\infty}$ algebra $A$ we have in mind derivations of the algebra $\widehat{\operatorname{cobar}} A=\left(\hat{T}\left(W^{*}\right), Q\right)$ (vector fields on a formal non-

\footnotetext{
${ }^{26}$ This means that the inner product does not vanish only if the sum of degrees of arguments is equal to $n$. For example, the odd bilinear form in pure spinor formalism of SYM can be considered as inner product of degree 3 .
} 
commutative manifold) that commute with $Q$; see equation (111). We say that symmetries $q_{1}, \ldots, q_{k}$ form Lie algebra $\mathfrak{g}$ if they satisfy commutation relations of $\mathfrak{g}$ up to $Q$-exact terms. These symmetries determine a homomorphism of Lie algebra $\mathfrak{g}$ into Lie algebra $\widehat{\mathrm{HH}}(A, A)$. We shall say that this homomorphism specifies weak action of $\mathfrak{g}$ on $A$.

In the case when $A_{\infty}$ algebra is $\mathbb{Z}$-graded we can impose the condition that the symmetry is compatible with the grading.

Another way to define symmetries of $A_{\infty}$ algebra is to identify them with $\mathrm{L}_{\infty}$ actions of Lie algebra $\mathfrak{g}$ on this algebra, i.e. with $\mathrm{L}_{\infty}$ homomorphisms of $\mathfrak{g}$ into differential Lie algebra of derivations $V$ ect of the algebra $\widehat{\operatorname{cobar}} A$ (the differential acts on $V$ ect as (super)commutator with $Q$ ). More explicitly $\mathrm{L}_{\infty}$ action is defined as a linear map

$$
q: \mathrm{Sym} \Pi \mathfrak{g} \rightarrow \Pi V e c t
$$

or as an element of odd degree

$$
q \in C^{\bullet}(\mathfrak{g}) \otimes V e c t
$$

obeying

$$
d_{\mathfrak{g}} q+[Q, q]+\frac{1}{2}[q, q]=0 .
$$

where $d_{\mathfrak{g}}$ is a differential entering the definition of Lie algebra cohomology. We can write $q$ in the form

$$
q=\sum \frac{1}{r !} q_{\alpha_{1}, \ldots, \alpha_{r}} c^{\alpha_{1}} \cdots c^{\alpha_{r}}
$$

where $c^{\alpha}$ are ghosts of the Lie algebra; here $d_{\mathfrak{g}}=\frac{1}{2} f_{\beta \gamma}^{\alpha} c^{\beta} c^{\gamma} \frac{\partial}{\partial c^{\alpha}}$ where $f_{\alpha \beta}^{\gamma}$ denote structure constants of $\mathfrak{g}$.

One can represent (121) as an infinite sequence of equations for the coefficients; the first of these equations has the form

$$
\left[q_{\alpha}, q_{\beta}\right]=f_{\alpha \beta}^{\gamma} q_{\gamma}+\left[Q, q_{\alpha \beta}\right]
$$

We see that $q_{\alpha}$ satisfy commutation relations of $\mathfrak{g}$ up to $Q$-exact terms (as we have said this means that they specify a weak action of $\mathfrak{g}$ on $A$ and a homomorphism $\mathfrak{g} \rightarrow \widehat{\mathrm{HH}}(A, A))$. 
In the remaining part of this section we use the notation $\mathrm{HH}$ instead of $\widehat{\mathrm{HH}}$.

Let us consider now an $\mathrm{A}_{\infty}$ algebra $A$ equipped with $\mathrm{L}_{\infty}$ action of Lie algebra $\mathfrak{g}$. To describe infinitesimal deformations of $A$ preserving the Lie algebra of symmetries we should find solutions of equations (121) and $[Q, Q]=0$ where $Q$ is replaced by $Q+\delta Q$ and $q$ by $q+\delta q$. After appropriate identifications these solutions can be described by elements of cohomology group that will be denoted by $\operatorname{HH}_{\mathfrak{g}}(A, A)$. To define this group we introduce ghosts $c^{\alpha}$. In other words we multiply $V e c t(\mathbb{V})$ by $\Lambda\left(\Pi \mathfrak{g}^{*}\right)$ and define the differential by the formula

$$
d=\widetilde{Q}+\frac{1}{2} f_{\beta \gamma}^{\alpha} c^{\beta} c^{\gamma} \frac{\partial}{\partial c^{\alpha}}+q_{\alpha} c^{\alpha}+\ldots
$$

The dots denote the terms having higher order with respect to $c^{\alpha}$. They should be included to satisfy $d^{2}=0$ if $q_{\alpha}$ obey commutations of $\mathfrak{g}$ up to $Q$-exact term. They can be expressed in terms of $q_{\alpha_{1}, \ldots, \alpha_{r}}$ :

$$
d=\widetilde{Q}+\frac{1}{2} f_{\beta \gamma}^{\alpha} c^{\beta} c^{\gamma} \frac{\partial}{\partial c^{\alpha}}+\sum_{r \geq 1} \frac{1}{r !} c^{\alpha_{1}} \cdots c^{\alpha_{r}} q_{\alpha_{1}, \ldots, \alpha_{r}}
$$

In the terminology introduced in Section $6 \mathrm{HH}_{\mathfrak{g}}(A, A)$ is the Lie algebra cohomology of $\mathfrak{g}$ with coefficients in the $\mathrm{L}_{\infty}$ differential $\mathfrak{g}$-module $(\operatorname{Vect}(\mathbb{V}), \widetilde{Q})$ :

$$
\mathrm{HH}_{\mathfrak{g}}(A, A)=H(\mathfrak{g},(\operatorname{Vect}(\mathbb{V}), \widetilde{Q}))
$$

From other side in the case of trivial $\mathfrak{g}$ we obtain Hochschild cohomology. Therefore we shall use the term Lie- Hochschild cohomology for the group (124).

Every deformation of $\mathrm{A}_{\infty}$ algebra $A$ induces a deformation of the algebra $\tilde{A}$ and of the corresponding $\mathrm{L}_{\infty}$ algebra $L_{N}(\tilde{A})$; if $\mathrm{A}_{\infty}$ algebra has Lie algebra of symmetries $\mathfrak{g}$ then the same is true for this $\mathrm{L}_{\infty}$ algebra. Deformations of $A_{\infty}$ algebra preserving the symmetry algebra $\mathfrak{g}$ induce symmetry preserving deformations of the $\mathrm{L}_{\infty}$ algebra 27. This remark permits us to say that the calculations of symmetry preserving deformations of $\mathrm{A}_{\infty}$ algebra $A$ corresponding to YM theory induces symmetry preserving deformations of EM for YM theories with gauge group $\mathrm{U}(N)$ for all $N$.

\footnotetext{
${ }^{27}$ At the level of cohomology groups it means that we have a series of homomorphisms $\mathrm{HH}_{\mathfrak{g}}(A, A) \rightarrow \mathrm{H}_{\mathfrak{g}}\left(L_{N}(\tilde{A}), L_{n}(\tilde{A})\right)$
} 
The calculation of cohomology groups $\operatorname{HH}_{\mathfrak{s u s y}}(Y M, Y M)$ permits us to describe SUSY-invariant deformations of EM. However we would like also to characterize Lagrangian deformations of EM. This problem also can be formulated in terms of homology. Namely we should consider $\mathrm{A}_{\infty}$ algebras with invariant inner product and their deformations. We say that $\mathrm{A}_{\infty}$ algebra $A$ is equipped with odd invariant nondegenerate inner product $\langle.,$.$\rangle if \left\langle a_{0}, \mu_{n}\left(a_{1}, \ldots, a_{n}\right)\right\rangle=$ $(-1)^{n+1}\left\langle a_{n}, \mu_{n}\left(a_{0}, \ldots, a_{n-1}\right)\right\rangle$. It is obvious that the corresponding $\mathrm{L}_{\infty}$ algebras $L_{N}(A)$ are equipped with odd invariant inner product. Therefore the corresponding vector field $Q$ comes from a solution of a Master equation $\{S, S\}=0$ (i.e. we have Lagrangian equations of motion). We shall check that the deformations of $\mathrm{A}_{\infty}$ algebra preserving invariant inner product are labeled by cyclic cohomology of the algebra 38 .

As we have seen the deformations of $\mathrm{A}_{\infty}$ algebra are labeled by Hochschild cohomology cocycles of differential $\widetilde{Q}$ (see formula (114)) acting on the space of derivations $\operatorname{Vect}(\mathbb{V})$.

A derivation $\rho$ is uniquely defined by its values on generators of the basis of vector space $W^{*}$ ( on generators of algebra $\left.\hat{T}\left(W^{*}\right)\right)$. Let us introduce notations $\rho\left(z^{i}\right)=\rho^{i}\left(z^{1}, \ldots, z^{n}\right)$. The condition that $\rho$ specifies a cocycle of $d$ means that it specifies a Hochschild cocycle with coefficients in $A$. The condition that $\rho$ preserves the invariant inner product is equivalent the cyclicity condition on $\rho_{i_{0}, i_{1} \ldots i_{n}}$, where $\rho_{i_{0}}\left(z^{1}, \ldots, z^{n}\right)=\sum \rho_{i_{0}, i_{1} \ldots i_{k}} z^{i_{1}} \ldots z^{i_{k}}$. (We lower the the upper index in $\rho$ using the invariant inner product.) The cyclicity condition has the form

$$
\rho_{i_{0}, i_{1} \ldots i_{k}}=(-1)^{k+1} \rho_{i_{k}, i_{0} \ldots i_{k-1}}
$$

We say that $\rho_{i_{0}, i_{1} \ldots, i_{k}}$ obeying formula (125) is a cyclic cochain. To define cyclic cohomology we use Hochschild differential on the space of cyclic cochains.

\footnotetext{
${ }^{28}$ One can say that the vector field $\rho$ preserving inner product is a Hamiltonian vector field. The cyclic cochain $\rho_{i_{0}, i_{1} \ldots i_{k}}$ can be considered as its Hamiltonian. The differential (114) acts on the space of Hamiltonian vector fields. The cohomology of corresponding differential acting on the space of Hamiltonians is called cyclic cohomology.
} 
If we consider deformations of $\mathrm{A}_{\infty}$ algebra with inner product and Lie algebra $\mathfrak{g}$ of symmetries and we are interested in deformations of $A$ to an algebra that also has invariant inner product and the same algebra of symmetries we should consider cyclic cohomology $\mathrm{HC}_{\mathfrak{g}}(A)$. The definition of this cohomology can be obtained if we modify the definition of $\operatorname{HC}(A)$ in the same way as we modified the definition of $\operatorname{HH}(A, A)$ to $\operatorname{HH}_{\mathfrak{g}}(A, A)$.

It is obvious that there exist a homomorphism from $\operatorname{HC}(A)$ to $\operatorname{HH}(A, A)$ and from $\operatorname{HC}_{\mathfrak{g}}(A)$ to $\operatorname{HH}_{\mathfrak{g}}(A, A)$ (every deformation preserving inner product is a deformation) 29 Our main goal is to calculate the image of $\operatorname{HC}_{\mathfrak{g}}(A)$ in $\mathrm{HH}_{\mathfrak{g}}(A, A)$ for the case of $\mathrm{A}_{\infty}$ algebra of YM theory, i.e. we would like to describe all supersymmetric deformations of YM that come from a Lagrangian.

Cyclic cohomology are related to Hochschild cohomology by Connes exact sequence:

$$
\ldots \rightarrow \mathrm{HC}^{n}(A) \rightarrow \operatorname{HH}^{n}\left(A, A^{*}\right) \rightarrow \mathrm{HC}^{n-1}(A) \rightarrow \mathrm{HC}^{n+1}(A) \rightarrow \ldots
$$

Similar sequence exists for Lie-cyclic cohomology.

To define the cyclic homology HC. $(A)$ we work with cyclic chains (elements of $A \otimes \ldots \otimes A$ factorized with respect to the action of cyclic group). The natural map of Hochschild chains with coefficients in $A$ to cyclic chains commutes with the differential and therefore specifies a homomorphism $\mathrm{HH}_{k}(A, A) \stackrel{l}{\rightarrow} \mathrm{HC}_{k}(A)$. This homomorphism enters the homological version of Connes exact sequence

$$
\ldots \rightarrow \mathrm{HC}_{n-1}(A) \stackrel{b}{\rightarrow} \mathrm{HH}_{n}(A, A) \stackrel{I}{\rightarrow} \mathrm{HC}_{n}(A) \stackrel{S}{\rightarrow} \mathrm{HC}_{n-2}(A) \rightarrow \ldots
$$

We define the differential $B: \operatorname{HH}_{n}(A, A) \rightarrow \operatorname{HH}_{n+1}(A, A)$ as a composition $b \circ I$.

An interesting refinement of Connes exact sequence exists in the case when $A$ is the universal enveloping of a Lie algebra $\mathfrak{g}$ over $\mathbb{C}$. In this case cyclic

\footnotetext{
${ }^{29}$ Notice that we have assumed that $A$ is equipped with non-degenerate inner product. The definition of cyclic cohomology does not require the choice of inner product; in general there exists a homomorphism $\mathrm{HC}(A) \rightarrow \operatorname{HH}\left(A, A^{*}\right)$. The homomorphism $\mathrm{HC}(A) \rightarrow \mathrm{HH}(A, A)$ can be obtained as a composition of this homomorphism with a homomorphism $\operatorname{HH}\left(A, A^{*}\right) \rightarrow$ $\mathrm{HH}(A, A)$ induced by a map $A^{*} \rightarrow A$.
} 
homology get an additional index: $\mathrm{HC}_{k, j}(A)$. Such groups fit into the long exact sequence [32]:

$\ldots \rightarrow \mathrm{HC}_{n-1, i}(U(\mathfrak{g})) \stackrel{b_{n-1, i}}{\rightarrow} \mathrm{HH}_{n}\left(U(\mathfrak{g}), \operatorname{Sym}^{i}(\mathfrak{g})\right) \stackrel{I_{n, i}}{\rightarrow} \mathrm{HC}_{n, i+1}(U(\mathfrak{g})) \stackrel{S_{n, i+1}}{\rightarrow} \mathrm{HC}_{n-2, i}(U(\mathfrak{g})) \rightarrow \ldots$

The differential

$$
B_{i}: \operatorname{HH}_{n}\left(U(\mathfrak{g}), \operatorname{Sym}^{i}(\mathfrak{g})\right) \rightarrow \operatorname{HH}_{n+1}\left(U(\mathfrak{g}), \operatorname{Sym}^{i-1}(\mathfrak{g})\right)
$$

is defined as a composition $b_{n, i+1} \circ I_{n, i}$. Finally if the Lie algebra $\mathfrak{g}$ is graded then all homological constructs acquire an additional bold index: $\operatorname{HH}_{n l}\left(U(\mathfrak{g}), \operatorname{Sym}^{i}(\mathfrak{g})\right)$, $\mathrm{HC}_{n, i, l}(U(\mathfrak{g}))$. This index is preserved by the differential in the above sequence.

It is worthwhile to mention that all natural constructions that exist in cyclic homology can be extended to Lie-cyclic homology.

It is important to emphasize that homology and cohomology theories we considered in this section are invariant with respect to quasi-isomorphism (under certain conditions that are fulfilled in our situation). 30

According to [26] a quasi-isomorphism of two algebras $A \rightarrow B$ induces an isomorphism in Hochschild cohomology $\mathrm{HH}^{\bullet}(A, A) \cong \mathrm{HH}^{\bullet}(B, B)$. As we have mentioned Hochschild cohomology $\mathrm{HH}^{\bullet}(A, A)$ is equipped with a structure of super Lie algebra, the isomorphism is compatible with this structure.

This theorem guarantees that quasi-isomorphism $A \rightarrow B$ allows us to translate a weak $\mathfrak{g}$ action from $A$ to $B$.

We have defined $\mathrm{L}_{\infty}$ action as an $\mathrm{L}_{\infty}$ homomorphism of Lie algebra $\mathfrak{g}$ into differential Lie algebra of derivations $\operatorname{Vect}(A)$. It follows from the results of [26] that a quasiisomorphism $\phi: A \rightarrow B$ induces a quasi- isomorphism $\tilde{\phi}$ : $\operatorname{Vect}(A) \rightarrow \operatorname{Vect}(B)$ compatible with $\mathrm{L}_{\infty}$ structure. 31 We obtain that $\mathrm{L}_{\infty}$ action on $A$ can be transferred to an $\mathrm{L}_{\infty}$ action on quasi-isomorphic algebra $B$.

\footnotetext{
${ }^{30}$ The most general results and precise formulation of this statement can be found in [26] for Hoschschild cohomology and in 27] for cyclic cohomology.

31 In fact the structure of $\operatorname{Vect}(A)$ is richer: it is a $\mathrm{B}_{\infty}$ algebra (see [26] for details), but we shall use only $\mathrm{L}_{\infty}$ (Lie) structure. One of the results of [26] asserts that $\tilde{\phi}$ is compatible with $\mathrm{B}_{\infty}$ structure. As a corollary it induces a quasi-isomorphism of $\mathrm{L}_{\infty}$ structures.
} 
The calculation of cohomology groups we are interested in is a difficult problem. To solve this problem we apply the notion of duality of associative and $\mathrm{A}_{\infty}$ algebras.

\section{B Duality}

We define a pairing of two differential graded augmented 32 algebras $A$ and $B$ as a degree one element $e \in A \otimes B$ that satisfies Maurer-Cartan equation

$$
\left(d_{A}+d_{B}\right) e+e^{2}=0
$$

Here we understand $A \otimes B$ as a completed tensor product.

Example 26 Let $x_{1}, \ldots, x_{n}$ be the generating set of the quadratic algebra $A$. The set $\xi^{1}, \ldots, \xi^{n}$ generates the dual quadratic algebra $A^{!}$(see preliminaries). The element $e=x_{i} \otimes \xi^{i}$ has degree one, provided $x_{i}$ and $\xi^{i}$ have degrees two and minus one. The element e satisfies $e^{2}=0$ - a particular case of (126) for algebras with zero differential and therefore specifies a pairing between $A$ and $A^{!}$.

Notice that the grading we are using here differs from the grading in the Section 2,

Remark. Many details of the theory depend on the completion of the tensor product, mentioned in the definition of $e$. We, however, chose to completely ignore this issue because the known systematic way to deal with it requires introduction of a somewhat artificial language of co-algebras. 33

We call a non-negatively (non-positively) graded differential algebra $A=$ $\bigoplus_{i} A_{i}$ connected, if $A_{0} \cong \mathbb{C}$. Such algebra is automatically augmented $\epsilon: A \rightarrow$

\footnotetext{
${ }^{32} \mathrm{~A}$ differential graded algebra $A$ is called augmented if it is equipped with a $d$-invariant homomorphism $\epsilon: A \rightarrow \mathbb{C}$ of degree zero. We assume that the algebras at hand are $\mathbb{Z}$-graded and graded components are finite-dimensional.

${ }^{33}$ One can define the notion of duality between algebra and co-algebra. This notion has better properties than the duality between algebras.
} 
$A_{0}$. We call a non-negatively graded connected algebra $A$ simply-connected if $A_{1} \cong 0$.

Let us consider a differential graded algebra $\operatorname{cobar} A=\left(T\left(\Pi A^{*}\right), d\right)$ where $A$ is an associative algebra and $d$ is the Hochschild differential. In other words we consider the co-bar construction for the algebra $A$.

Proposition 27 The pairing e defines the map

$$
\rho: \operatorname{cobar}(A) \rightarrow B
$$

of differential graded algebras.

Proof. The algebra cobar $(A)$ is generated by elements of $\Pi A^{*}$. The value of the map $\rho$ on $l \in \Pi A^{*}$ is equal to

$$
\rho(l)=\left\langle l, f_{i}\right\rangle g^{i}
$$

where $e=f_{i} \otimes g^{i} \in A \otimes B$. The compatiblity of $\rho$ with the differential follows automatically from (126). (Notice, that for graded spaces we always consider the dual as graded dual, i.e. as a direct sum of dual spaces to the graded components.)

Similarly the element $e$ defines a map $\operatorname{cobar}(B) \rightarrow A$.

Definition 28 The differential algebras $A$ and $B$ are dual if there exists a pairing $(A, B, e)$ such that the maps $\operatorname{cobar}(A) \rightarrow B$ and $\operatorname{cobar}(B) \rightarrow A$ are quasiisomorphisms.

34

Notice that duality is invariant with respect to quasi-isomorphism.

If $A$ is quadratic then $A$ is dual to $A^{!}$iff $A$ is a Koszul algebra.

If a differential graded algebra $A$ has a dual algebra, then $A$ is dual to $\operatorname{cobar} A$. If $A$ is a connected and simply-connected differential graded algebra, i.e. $A=\bigoplus_{i \geq 0} A_{i}$ and $A_{0}=\mathbb{C}$ and $A_{1}=0$, then $A$ and cobar $A$ are dual.

\footnotetext{
${ }^{34}$ Very similar notion of duality was suggested independently by Kontsevich [28.
} 
If differential graded algebras $A$ and $B$ are dual it is clear that Hochschild cohomology $\mathrm{HH}(A, \mathbb{C})$ of $A$ with trivial coefficients coincide with intrinsic cohomology of $B$. This is because $B$ is quasi-isomorphic to cobar $(A)$. One can say also that

$$
\operatorname{HH}(A, A)=\operatorname{HH}(B, B),
$$

This is clear because these cohomology can be calculated in terms of complex $A \otimes B$, that is quasi-isomorphic both to $A \otimes \operatorname{cobar} A$ and $\operatorname{cobar} B \otimes B$. Notice, that the isomorphism (127) does not preserve the grading.

This statement can be generalized to Hochschild cohomology of $A$ with coefficients in any bimodule $M$. Namely, we should introduce in $B \otimes M$ a differential by the formula

$$
d(b \otimes m)=\left(d_{B}+d_{M}\right) b \otimes m+[e, b \otimes m]
$$

Proposition 29 Let $A$ be a connected and simply-connected differential graded algebra, i.e. $A=\bigoplus_{i \geq 0} A_{i}$ and $A_{0}=\mathbb{C}$ and $A_{1}=0$. Then the Hochschild cohomology $\mathrm{HH}(A, M)$ coincide with the cohomology of $B \otimes M$ with respect to differential (128).

To prove this statement we notice that the quasi-isomorphism cobar $A \rightarrow B$ induces a homomorphism $C(A, M)=\operatorname{cobar} A \otimes M \rightarrow B \otimes M$; it follows from (118) that this homomorphism commutes with the differentials and therefore induces a homomorphism on homology. The induced homomorphism is an isomorphism; this can be derived from the fact that the map cobar $A \rightarrow B$ is a quasi-isomorphism. (The derivation is based on the techniques of spectral sequences; the condition on algebra $A$ guarantees the convergence of spectral sequence.)

The above proposition can be applied to the case when $A$ is a Koszul quadratic algebra and $B=A^{!}$is the dual quadratic algebra. We obtain the following statement generalizing the proposition 3 in Section 2.2. (There is also a statement for Hochschild homology generalizing proposition 4 ) 
Proposition 30 [39] We assume that A is Koszul. The Hochschild cohomology $\mathrm{HH}^{\bullet}(A, N)$ is equal to the cohomology of the complex $N_{c} \stackrel{\text { def }}{=} N \otimes A^{!}$. The differential is the graded commutator with e. The complex $N_{c}$ splits according to degree:

$$
N_{c \boldsymbol{m}}^{\bullet}=N_{m} \otimes A_{0}^{!} \rightarrow N_{m+1} \otimes A_{1}^{!} \rightarrow \ldots
$$

The complex $N_{c \boldsymbol{m}}^{\bullet}$ is defined for positive and negative $\boldsymbol{m}$, we assume that $N_{m}=$ 0 if $m<m_{0}$. Then $\mathrm{HH}^{k, \boldsymbol{m}}(A, N)=H^{k}\left(N_{c}^{\bullet} \boldsymbol{m}\right)$.

Proposition 31 [39] We assume that $A$ is Koszul. Homology HH. $(A, N)$ are equal to the cohomology of the complex $N_{h} \stackrel{\text { def }}{=} N \otimes A^{! *}$. The space $A^{! *}=$ $\bigoplus_{n \geq 0} A_{n}^{! *}$ is an $A^{!}$-bimodule dual to $A^{!}$. The differential is a commutator with e given by the formula (25). The complex $N_{h}$ splits :

$$
N_{h \boldsymbol{m}}^{\bullet}=N_{m_{0}} \otimes A_{m-m_{0}}^{! *} \stackrel{d}{\rightarrow} \ldots N_{0} \otimes A_{m}^{! *} \stackrel{d}{\rightarrow} \ldots N_{m-1} \otimes A_{1}^{! *} \stackrel{d}{\rightarrow} N_{m} \otimes A_{0}^{! *}
$$

Then $\operatorname{HH}_{k, \boldsymbol{m}}(A, N)=H^{m-k}\left(N_{h}^{\bullet} \boldsymbol{m}\right)$.

Proposition 3 follows from Propositions 30,31 if we set $A=U(\mathfrak{g}), A^{!}=\mathcal{C}$ and use the fact that Lie algebra cohomology of $\mathfrak{g}$ with coefficients in a $\mathfrak{g}$-module coincide with Hochschild cohomology of $U(\mathfrak{g})$ with coefficients in $U(\mathfrak{g})$-bimodule. 35

Proposition 32 If differential graded algebra $A$ is dual to $B$ and quasi-isomorphic to the envelope $U(\mathfrak{g})$ of Lie algebra $\mathfrak{g}$ then $B$ is quasi-isomorphic to the supercommutative differential algebra $C^{\bullet}(\mathfrak{g})$.

\footnotetext{
${ }^{35}$ Let $N$ be a $U(\mathfrak{g})$-bimodule. Define a new structure of $\mathfrak{g}$-module on $N$ by the formula $l \otimes n \rightarrow l n-n l, l \in \mathfrak{g}, n \in N$ There is an isomorphism [10]

$$
\operatorname{HH}^{i}(U(\mathfrak{g}), N) \rightarrow \mathrm{H}^{i}(\mathfrak{g}, N),
$$

defined by the formula:

$$
\gamma\left(l_{1}, \ldots, l_{n}\right) \rightarrow \tilde{\gamma}=\frac{1}{n !} \sum_{\sigma \in S_{n}} \pm \gamma\left(l_{\sigma(1)}, \ldots, l_{\sigma(n)}\right), l_{i} \in \mathfrak{g}
$$

. There is a similar isomorphism for homology. 
This statement follows from the fact that the cohomology of $C^{\bullet}(\mathfrak{g})$ (=Lie algebra cohomology of $\mathfrak{g})$ coincides with Hochschild cohomology of $U(\mathfrak{g})$ with trivial coefficients.

It turns out that it is possible to calculate cyclic and Hochschild cohomology of $A$ in terms of suitable homological constructions for a dual algebra $B$.

Let $A$ and $B$ be dual differential graded algebras. Let us assume that $A$ and $B$ satisfy assumptions of Proposition 29.

Proposition 33 Under above assumptions there is a canonical isomorphism

$$
\mathrm{HC}_{-1-n}(A) \cong \mathrm{HC}^{n}(B),
$$

where $\mathrm{HC}^{n}\left(\mathrm{HC}_{n}\right)$ stands for $i$-th cyclic cohomology(resp. homology) of an algebra.

Proposition 34 Under the above assumptions there is an isomorphism

$$
\mathrm{HH}^{n}\left(A, A^{*}\right)=\mathrm{HH}_{-n}(B, B),
$$

where $\mathrm{HH}^{n}\left(\mathrm{HH}_{n}\right)$ stands for $n$-th Hochschild cohomology (resp. homology).

For the case when $A$ and $B$ are quadratic algebras these two propositions were proven in [22]. The proof in general case is similar. It can be based on results of [9] or [32].

Let us illustrate some of above theorems on concrete examples. The algebra $\mathcal{S}$ is dual to $U(L)$. This means, that

$$
\mathrm{HH}^{\bullet}(\mathcal{S}, \mathcal{S})=\mathrm{HH}^{\bullet}(U(L), U(L))=H^{\bullet}(L, U(L))
$$

The reduced Berkovits algebra $B_{0}$ is dual to $U(Y M)$, hence

$$
\mathrm{HH}^{\bullet}\left(B_{0}, B_{0}\right)=\mathrm{HH}^{\bullet}(U(Y M), U(Y M))=\mathrm{H}^{\bullet}(Y M, U(Y M))
$$

We need the following information about this cohomology ([36]):

$$
\begin{aligned}
& \mathrm{H}^{0}(Y M, U(Y M)) \cong \mathbb{C} \\
& \mathrm{H}^{1}(Y M, U(Y M)) \cong \mathbb{C}+S^{*}+\Lambda^{2}(V)+V+S^{*}
\end{aligned}
$$


Notice, that the answer for $\mathrm{H}^{1}(Y M, U(Y M))=\mathrm{HH}^{1}(U(Y M), U(Y M))$ has clear physical interpretation: symmetries of SYM theory (translations, Lorenz transformations and supersymmetries) specify derivations of the algebra $U(Y M)$.

Representing $U(Y M)$ as $\operatorname{Sym}(Y M)$ we obtain additional grading on cohomology:

$$
\begin{array}{r}
\mathrm{H}^{1}\left(Y M, \operatorname{Sym}^{1}(Y M)\right) \cong \mathbb{C}+S^{*}+\Lambda^{2}(V) \\
\mathrm{H}^{1}\left(Y M, \operatorname{Sym}^{0}(Y M)\right) \cong V+S^{*} \\
\mathrm{H}^{1}\left(Y M, \operatorname{Sym}^{i}(Y M)\right)=0, i \geq 2
\end{array}
$$

It follows from the remarks in Appendix A that $\mathrm{H}^{k}(Y M, U(Y M))=\mathrm{HH}^{k}(U(Y M), U(Y M))=$ 0 for $k>3$.

As we mentioned in Section 6 the odd symplectic structure in pure spinor formalism is specified by degenerate closed two-form $\omega$. This form determines an odd inner product of degree 3 on $B_{0}$ that generates Poincaré isomorphism $\mathrm{H}^{i}(Y M, U(Y M)) \stackrel{P}{\cong} \mathrm{H}_{3-i}(Y M, U(Y M))$.

\section{On the relation of the Lie algebra and the BV approaches to the deformation problem}

Our main goal is to calculate SUSY deformations of 10D YM theory and its reduction to a point. In Section 4 we have reduced this question to a homological problem. Another reduction of this kind comes from BV formalism (Section [6 and Appendix (A). Here we shall relate these two approaches. For simplicity we shall talk mostly about the reduced case; we shall describe briefly the modifications that are necessary in the unreduced case.

We shall use the fact that under certain conditions all objects we are interested in are invariant with respect to quasi- isomorphisms.

We can study the symmetries of Yang-Mills theory using the $\mathrm{A}_{\infty}$ algebra $\mathcal{A}$ constructed in Appendix $\mathrm{A}$ or any other algebra that is quasi-isomorphic to $\mathcal{A}$. In BV formalism a Lie algebra action should be replaced by weak action 
or by $\mathrm{L}_{\infty}$ action. It will be important for us to work with $\mathrm{L}_{\infty}$ action, because this action is used in the construction of formal deformations (Section 7). We consider the case of YM theory dimensionally reduced to a point ; in this case we use the notation $\mathcal{A}=b v_{0}$ and the algebra cobarb $v_{0}=B V_{0}$ is quasi-isomorphic to $U(Y M)$ (to the envelope of Lie algebra $Y M$ ); see [35], Theorem 1. 36

The algebra $b v_{0}$ is dual to the algebra $B V_{0}$. This means that $b v_{0}$ is quasiisomorphic to $C^{\bullet}(Y M)$ (to the differential commutative algebra that computes Lie algebra cohomology with trivial coefficients; see Appendix B] (32)).

One can construct an $\mathrm{L}_{\infty}$ action of the reduced supersymmetry algebra $\mathfrak{g}=\Pi \mathbb{C}^{16}$ on the algebras $b v_{0}, C^{\bullet}(Y M), U(Y M)$. It is sufficient to construct such an action on one of these algebras.

Let us describe the action on $C^{\bullet}(Y M)$.

We shall use the Lie algebra $L$ defined in Section 2. By construction $L$ as a linear space is isomorphic to the direct sum $S+Y M$, where $S=L^{1}$ is spanned by $\boldsymbol{\theta}_{1}, \ldots, \boldsymbol{\theta}_{16}$. Thus

$$
C^{\bullet}(L) \cong \mathbb{C}\left[\left[t^{1}, \ldots, t^{16}\right]\right] \otimes C^{\bullet}(Y M)
$$

where $t^{\alpha}$ are even variables dual to $\boldsymbol{\theta}_{\alpha}$. The differential $d_{L}$ in $C^{\bullet}(L)$ is the sum

$$
d_{L}=d_{Y M}+q,
$$

where $q$ is equal to $t^{\alpha} t^{\beta} q_{\alpha \beta}+t^{\gamma} q_{\gamma}$. The operators $q_{\alpha \beta}, q_{\gamma}$ are derivations of $C^{\bullet}(Y M)$. We can interpret $q$ as map of $\operatorname{Sym}(\Pi \mathfrak{g})=\operatorname{Sym}\left(\mathbb{C}^{16}\right)$ into the space of derivations of $C^{\bullet}(Y M)$. It is easy to check, that this map obeys (121); hence it specifies $\mathrm{L}_{\infty}$ action of $\mathfrak{g}=\Pi \mathbb{C}^{16}$ on $C^{\bullet}(Y M)$.

Another way to describe this $\mathrm{L}_{\infty}$ action of $\mathfrak{g}=\Pi \mathbb{C}^{16}$ is to construct the corrections that arise because the dimensionally reduced supersymmetries $q_{\gamma}$ defined by the formula $q_{\gamma}=[\boldsymbol{\theta}, x]$ anti- commute only on-shell. The operators

\footnotetext{
${ }^{36}$ The algebra $B V_{0}$ has as generators the generators of $U(Y M)$, corresponding antifields and $c^{*}$ (the antifield for ghost) ; sending antifields and $c^{*}$ to zero we obtain a homomorphism of differential algebras. (Recall that the differential on $U(Y M)$ is trivial.) It has been proven in 35 that this homomorphism is a quasi-isomorphism.
} 
$q_{\alpha \beta}$ can be interpreted as $\mathrm{L}_{\infty}$ corrections to the action of the Lie algebra $\mathfrak{g}=$ $\Pi \mathbb{C}^{16}$. In this construction no higher order operators $q_{\alpha_{1}, \ldots, \alpha_{n}}(n \geq 3)$ are present.

We should say a word of caution. The action of $\Pi \mathbb{C}^{16}$ on $b v_{0}$ constructed this way could be incompatible with the inner product. A refined version of this action, free from the shortcoming, is constructed in Appendix D.

Similar arguments permit us to construct an $\mathrm{L}_{\infty}$ action of SUSY Lie algebra in unreduced case. In this case the algebra $\mathcal{A}$ is denoted by $b v$, cobarbv $=B V$ is quasi-isomorphic to $U(T Y M)$ and $b v$ is quasi- isomorphic to $C^{\bullet}(T Y M)$. To construct an $\mathrm{L}_{\infty}$ action of SUSY Lie algebra on $C^{\bullet}(T Y M)$ we notice that as a vector space $L$ can be represented as a direct sum of vector subspaces $L^{1}+L^{2}$ and $T Y M$. This means that

$$
C^{\bullet}(L) \cong \mathbb{C}\left[\left[t^{1}, \ldots, t^{16}\right]\right] \otimes \Lambda\left[\xi^{1}, \ldots, \xi^{10}\right] \otimes C^{\bullet}(T Y M)
$$

where $t^{\alpha}, \xi^{i}$ can be interpreted as even and odd ghosts of the Lie algebra $\mathfrak{s u s y .}$ Again we can construct the $\mathrm{L}_{\infty} \mathfrak{s u s y}$ action on $C^{\bullet}(T Y M)$ using the differential $d_{L}$ acting on $C^{\bullet}(L)$. Namely, we choose a basis $\left\langle e_{\gamma},\right\rangle \gamma \geq 1$ in $T Y M$, a basis $\left\langle\theta_{\alpha},\right\rangle \alpha=1, \ldots, 16$ in $S$ and $\left\langle v_{i}\right\rangle, i=1, \ldots, 10$ in $V$. Together they form a basis in $\left\langle e_{\gamma}, \theta_{\alpha}, v_{i}\right\rangle$ of $T Y M+S+V \cong L$. The structure constants of the Lie algebra in this basis are $\left[e_{\gamma}, e_{\gamma^{\prime}}\right]=\sum_{\delta \geq 0} c_{\gamma \gamma^{\prime}}^{\delta} e_{\delta},\left[\theta_{\alpha}, e_{\gamma}\right]=\sum_{k} f_{\alpha \gamma}^{\delta} e_{\delta},\left[v_{i}, e_{\gamma}\right]=\sum_{k} g_{i j}^{k} e_{\gamma}$, $\left[\theta_{\alpha}, \theta_{\beta}\right]=\Gamma_{\alpha \beta}^{i} v_{i},\left[\theta_{\alpha}, v_{i}\right]=\sum_{\delta} h_{\alpha i}^{\delta} e_{\delta}$. The algebra Sym $\Pi L^{*} \cong \mathbb{C}\left[\left[t^{1}, \ldots, t^{16}\right]\right] \otimes$ $\Lambda\left[\xi^{1}, \ldots, \xi^{10}\right] \otimes C^{\bullet}(T Y M)$ has generators $\epsilon^{\gamma}, t^{\alpha}, \xi_{i}$ dual and opposite parity to $e_{\gamma}, \theta_{\alpha}, v_{i}$. The differential can be written as

$$
\begin{aligned}
& c_{\gamma \gamma^{\prime}}^{\delta} \epsilon^{\gamma} \epsilon^{\gamma^{\prime}} \frac{\partial}{\partial \epsilon^{\delta}}+ \\
& +f_{\alpha \gamma}^{\gamma^{\prime}} t^{\alpha} \epsilon^{\gamma} \frac{\partial}{\partial \epsilon^{\gamma^{\prime}}}+ \\
& +g_{i \gamma}^{\gamma^{\prime}} \xi^{i} \epsilon^{\gamma} \frac{\partial}{\partial \epsilon^{\gamma^{\prime}}}+ \\
& +r_{i \alpha}^{\gamma} \xi^{i} t^{\alpha} \frac{\partial}{\partial \epsilon^{\gamma}}+\Gamma_{\alpha \alpha^{\prime}}^{i} t^{\alpha} t^{\alpha^{\prime}} \frac{\partial}{\partial \xi_{i}}
\end{aligned}
$$

We identify $c_{\gamma \gamma^{\prime}}^{\delta} \epsilon^{\gamma} \epsilon^{\gamma^{\prime}} \frac{\partial}{\partial \epsilon^{\delta}}$ with the differential in $C^{\bullet}(T Y M)$. All other terms define the desired $\mathrm{L}_{\infty}$ action. 
To classify infinitesimal SUSY deformations of reduced YM theory it is sufficient to calculate Lie- Hochschild cohomology $\operatorname{HH}_{\mathfrak{g}}\left(C^{\bullet}(Y M), C^{\bullet}(Y M)\right)$. First of all we notice that one can use duality between $C^{\bullet}(Y M)$ and $U(Y M)$ to calculate Hochschild cohomology

$\mathrm{HH}\left(C^{\bullet}(Y M), C^{\bullet}(Y M)\right)=\mathrm{HH}(U(Y M), U(Y M))=\mathrm{H}(Y M, U(Y M))=\mathrm{H}(Y M, \operatorname{Sym}(Y M))$.

Here we are using (127) and the relation between Hochschild cohomology of enveloping algebra $U(Y M)$ and Lie algebra cohomology of $Y M$ as well as Poincaré -Birkhoff-Witt theorem. Analyzing the proof of (135) we obtain quasiisomorphism between the complex $\operatorname{cobar} C^{\bullet}(Y M) \otimes C^{\bullet}(Y M)$ that we are using in calculation of $\operatorname{HH}\left(C^{\bullet}(Y M), C^{\bullet}(Y M)\right)$ and the complex $\operatorname{Sym}(\Pi Y M)^{*} \otimes \operatorname{Sym} Y M$ with cohomology $\mathrm{H}(Y M, \operatorname{Sym}(Y M))$.

To calculate the Lie-Hochschild cohomology $\operatorname{HH}_{\mathfrak{g}}\left(C^{\bullet}(Y M), C^{\bullet}(Y M)\right)$ we should consider a complex $C^{\bullet}(\mathfrak{g}) \otimes \operatorname{cobar} C^{\bullet}(Y M) \otimes C^{\bullet}(Y M)$ with the differential defined in (123). This complex is quasi-isomorphic to $C^{\bullet}(\mathfrak{g}) \otimes \operatorname{Sym}(\Pi Y M)^{*} \otimes$ Sym $Y M$ with appropriate differential.

Now we can notice that $C^{\bullet}(\mathfrak{g}) \otimes \operatorname{Sym}(\Pi Y M)^{*}=\mathbb{C}\left[\left[t^{1}, \ldots, t^{16}\right]\right] \otimes \operatorname{Sym}\left(\Pi Y M^{*}\right)=$ $\operatorname{Sym}\left(\Pi L^{*}\right)$. Hence we can reassemble $C^{\bullet}(\mathfrak{g}) \otimes \operatorname{Sym}(\Pi Y M)^{*} \otimes \operatorname{Sym} Y M$ into $\operatorname{Sym}\left(\Pi L^{*}\right) \otimes \operatorname{Sym}(Y M)$, which is isomorphic to $C^{\bullet}(L, U(Y M))$.

We see that Lie- Hochschild cohomology $\operatorname{HH}_{\mathfrak{g}}\left(C^{\bullet}(Y M), C^{\bullet}(Y M)\right)$ with $\mathfrak{g}=$ $\Pi \mathbb{C}^{16}$ classifying infinitesimal SUSY deformations in the reduced case is isomorphic to $\mathrm{H}^{\bullet}(L, U(Y M))$.

Lie-Hochschild cohomology $\operatorname{HH}_{\mathfrak{g}}\left(C^{\bullet}(T Y M), C^{\bullet}(T Y M)\right)$ where $\mathfrak{g}=\mathfrak{s u s y}$ govern SUSY deformations of unreduced SYM. Similar considerations permit us to prove that these cohomology are isomorphic to $\mathrm{H}^{\bullet}(L, U(T Y M))$.

The above statements agree with the theorems of Section 4 where it is proven that two-dimensional cohomology of $L$ with coefficients in $U(Y M)$ and in $U(T Y M)$ correspond to SUSY deformations in reduced and unreduced cases. We see that BV approach leads to wider class of SUSY deformations. 37 How-

\footnotetext{
37 One can modify the arguments of Section 4 to cover the additional deformations arising in
} 
ever, one can prove that all super Poincaré invariant deformations in the reduced case are covered by the constructions of Sections 4. The proof is based on the remark that the groups $\mathrm{H}^{i}(L, U(Y M))$ do not contain Spin(10)-invariant elements for $i>2$. (This remark can be derived from the considerations of Section [5]) Notice, however, that the group $\mathrm{H}^{i}(L, U(T Y M))$ contains $\operatorname{Spin}(10)$-invariant element for $i=3$. This element is responsible for the super Poincaré invariant deformation of $\mathrm{L}_{\infty}$ action of supersymmetry, but it generates a trivial infinitesimal variation of action functional. However, corresponding formal deformation constructed in Section 7 can be non-trivial.

\section{The $\mathrm{L}_{\infty}$ action of the supersymmetry algebra in the BV formulation}

In Appendix $\mathrm{C}$ we have shown that one can construct an $\mathrm{L}_{\infty}$ action of SUSY algebra on $b v$. In this section we shall give another proof of the existence of this action; we shall show that this proof permits us to construct an $\mathrm{L}_{\infty}$ action that is compatible with invariant inner product on $b v$. We use the formalism of pure spinors in our considerations.

The pure spinor construction will be preceded by a somewhat general discussion of $\mathrm{L}_{\infty}$-invariant traces.

Suppose that the tensor product $A \otimes \operatorname{Sym}(\Pi \mathfrak{g})$ is furnished with a differential $d$ which can be written as $d_{A}+d_{\mathfrak{g}}+q$, where $d_{A}$ is a differential in $A, d_{\mathfrak{g}}$ is the Lie algebra differential (86 ) in $\operatorname{Sym}(\Pi \mathfrak{g})$ and $q=\sum_{n \geq 1} \frac{1}{n !} c^{\alpha_{1}} \cdots c^{\alpha_{n}} q_{\alpha_{1}, \ldots, \alpha_{n}}$ is the generating function of derivations $q_{\alpha_{1}, \ldots, \alpha_{n}}$ that satisfies the analog of 121 ). We say that $A$ is equipped with $\mathfrak{g}$ - equivariant trace if there is a linear map

$$
p: A \otimes \operatorname{Sym}(\Pi \mathfrak{g}) \rightarrow \operatorname{Sym}(\Pi \mathfrak{g})
$$

which satisfies $p\left(\left[a, a^{\prime}\right]\right)=0$ and

$$
p\left(Q_{A}+d_{\mathfrak{g}}+q\right) a=d_{\mathfrak{g}} p a
$$

BV formalism. The modification is based on consideration of $\mathrm{A}_{\infty}$ deformations of associative algebras $U(Y M)$ and $U(T Y M)$. 
for every $a \in A \otimes \operatorname{Sym}(\Pi \mathfrak{g})$. In the case when we have an ordinary action of a Lie algebra $\mathfrak{g}$ on a differential graded Lie algebra $A$ and a trace functional $p$ is $\mathfrak{g}$-invariant, i.e. $p(l a)=0$ for any $l \in \mathfrak{g}$ and $a \in A$ then $p$ is trivially a $\mathfrak{g}$-equivariant functional.

This construction provides us with an inner product $\langle a, b\rangle=p_{\mathfrak{g}}(a b)$ on $A$ with values in $\operatorname{Sym}(\Pi \mathfrak{g})$ (ghost valued inner product). If the trace $p$ is odd the corresponding inner product is also odd.

In pure spinor formalism the algebra $S \otimes C^{\infty}\left(\mathbb{R}^{10 \mid 16}\right)$ is equipped with the differential $D$ given by the formula (17) and the $D$-closed odd linear functional

$$
p: S \otimes C^{\infty}\left(\mathbb{R}^{10 \mid 16}\right) \rightarrow \mathbb{C}
$$

It is defined on elements that decay sufficiently fast at the space-time infinity. The functional $p$ splits into a tensor product of translation-invariant volume form vol on $\mathbb{R}^{10}$ and a functional $p_{\text {red }}: S \otimes C^{\infty}\left(\mathbb{R}^{0 \mid 16}\right) \rightarrow \mathbb{C}$.

The super-symmetries generators are

$$
\theta_{\alpha}=\frac{\partial}{\partial \psi^{\alpha}}+\Gamma_{\alpha \beta}^{i} \psi^{\beta} \frac{\partial}{\partial x^{i}} .
$$

The functional $p_{\text {red }}$ is Spin(10)-invariant. Also it can be characterised as the only nontrivial Spin(10)- invariant functional on $S \otimes C^{\infty}\left(\mathbb{R}^{0 \mid 16}\right)$. This follows from simple representation theory for $\operatorname{Spin}(10)$. This fact enables us to construct an "explicit" formula for $p_{\text {red }}$. The projection

$$
\mathbb{C}\left[\lambda^{1}, \ldots, \lambda^{16}\right] \stackrel{k}{\rightarrow} S
$$

commutes with the action of Spin(10). A simple corollary of representation theory is that $k$ has a unique linear $\operatorname{Spin}(10)$-equivariant splitting $k^{-1}$. Using this splitting we can identify elements of $S$ with $\Gamma$-traceless elements in $\mathbb{C}\left[\lambda^{1}, \ldots, \lambda^{16}\right]$. Let us define a $\operatorname{Spin}(10)$-invariant differential operator on $\mathbb{C}\left[\lambda^{1}, \ldots, \lambda^{16}\right] \otimes$ $\Lambda\left[\psi^{1}, \ldots, \psi^{16}\right]$ by the formula

$$
P=\Gamma_{m}^{\alpha \beta} \frac{\partial}{\partial \lambda^{\alpha}} \frac{\partial}{\partial \psi^{\beta}} \Gamma_{n}^{\gamma \delta} \frac{\partial}{\partial \lambda^{\gamma}} \frac{\partial}{\partial \psi^{\delta}} \Gamma_{k}^{\epsilon \varepsilon} \frac{\partial}{\partial \lambda^{\epsilon}} \frac{\partial}{\partial \psi^{\varepsilon}} \Gamma_{m n k}^{\mu \nu} \frac{\partial}{\partial \psi^{\mu}} \frac{\partial}{\partial \psi^{\nu}}
$$

We define $p_{\varnothing}(a)$ as $\left.P a\right|_{\lambda, \psi=0}$. 
One of the properties of $p_{\varnothing}$ is that it is $D$-closed

$$
p_{\varnothing}(D a)=0 .
$$

It is not, however, invariant with respect to the action of supersymmetries. It satisfies a weaker condition

$$
p_{\varnothing}\left(\theta_{\alpha} a\right)=p_{\alpha}(D a)
$$

The generating function technique that was used for formulation of $\mathrm{L}_{\infty}$ action in the $\mathrm{BV}$ formulation can be used here. We have even ghosts $t^{1}, \ldots, t^{16}$ and odd ghosts $\xi^{1}, \ldots, \xi^{10}$. We define the total $\mathrm{L}_{\infty}$ action operator $D_{\infty}$ as the sum

$$
D_{\infty}=D+t^{\alpha} \theta_{\alpha}+\xi^{i} \frac{\partial}{\partial x^{i}}+d_{\mathfrak{s u s y}}
$$

The condition $D_{\infty}^{2}=0$ is equivalent to the standard package of properties of the pure spinor BV differential and supersymmetries.

We shall construct a generating function of functionals $p$ that satisfies equation

$$
p(a)=d_{\mathfrak{s u s y}} p(a)+\text { exact terms }
$$

We define a formal perturbation $p$ of $P$ as a composition

$$
p=\sum_{k \geq 0} \frac{1}{k !} P\left(t^{\alpha} \frac{\partial}{\partial \lambda^{\alpha}}\right)^{k}
$$

Then

$$
p_{\varnothing}(a) \text { is equal to }\left.p(a)\right|_{t, \psi=0}
$$

The $\mathrm{L}_{\infty}$-invariant trace functional can be defined as

$$
p_{\mathfrak{s u s y}}(a)=\int p(a) \mathrm{vol}
$$

The (ghost dependent) odd inner product corresponding to this trace is also $\mathrm{L}_{\infty}$ invariant. It specifies a homologically non-degenerate two-form on a formal $Q$-manifold; this two-form obeys the condition of proposition 25, hence we can apply the conclusion of this proposition to classify Lagrangian deformations of SYM theory. 


\section{E Calculation of the hypercohomology}

To justify the calculations of Section 5 we should check that the embedding $\mathcal{W}^{*} \rightarrow \mathcal{Y M}^{\bullet}$ and the embedding $\operatorname{Sym}^{i}\left(\mathcal{W}^{*}\right) \rightarrow \operatorname{Sym}^{i}(\mathcal{Y} \mathcal{M})$ are quasi-isomorpisms. In other words, we should prove that these homomorphisms induce isomorphisms of hypercohomology. We should prove also similar results for embedding $\mathcal{W} \rightarrow \mathcal{T Y \mathcal { M }}$ and embedding $\operatorname{Sym}^{i}(\mathcal{W}) \rightarrow \operatorname{Sym}^{i}(\mathcal{Y} \mathcal{M})$.

We shall start with some general considerations. As we have noticed in Section [5, there are two spectral sequences that can be used in calculation of hypercohomology of the complex of vector bundles $\mathcal{N}_{\boldsymbol{l}}$. Here we shall use the second one (with $E_{2}=H^{i}\left(H^{j}\left(\Omega\left(\mathcal{N}_{l}\right), d_{e}\right), \bar{\partial}\right)$.

First of all we shall consider the modules $N$ where $N=L, Y M$ or $T Y M$, corresponding differential vector bundles $\mathcal{N}=\mathcal{L}, \mathcal{Y} \mathcal{M}, \mathcal{T Y} \mathcal{M}$ and differential $P$-modules $N_{P}$ obtained as fibers of these bundles over the point $\lambda_{0} \in \mathcal{Q}$. The differential on the module $N_{P}$ is obtained as restriction of the differential $d_{e}$ on vector bundle $\mathcal{N}$ and will be denoted by the same symbol.

Let us start with calculation of the cohomology of the module $U(L)_{P}$.

Proposition $35 \mathrm{H}^{i}\left(U(L)_{P}, d_{e}\right)=\operatorname{HH}^{i}\left(S, \mathbb{C}_{\lambda_{0}}\right)$

Here one-dimensional $S$-bimodule $\mathbb{C}_{\lambda_{0}}$ is obtained by specialization at $\lambda_{0} \in \mathcal{C}$ with coordinates $\lambda_{0}^{\alpha}$. In more details the left and right actions of polynomial $f(\lambda)$ on generators $a \in \mathbb{C}_{\lambda_{0}}$ is given by the formula $f(\lambda) \times a=f\left(\lambda_{0}\right) a$

Proof. This is a direct application of Proposition 30 where $N=\mathbb{C}_{\lambda_{0}}$ and $A=\mathcal{S}, A^{!}=U(L)$.

To calculate RHS in Proposition 35 we use the following statement that can be considered as a weak form of Hochschild-Kostant-Rosenberg theorem (see [17):

Proposition 36 Suppose $A$ is a ring of algebraic functions on affine algebraic variety. Let $\mathbb{C}_{x}$ denote a one-dimensional bimodule, corresponding to a smooth point $x$. Then $\operatorname{HH}^{i}\left(A, \mathbb{C}_{x}\right)=\Lambda^{i}\left(T_{x}\right)$, where $T_{x}$ is the tangent space at $x$. 
Corollary $37 \mathrm{HH}^{i}\left(S, \mathbb{C}_{\lambda_{0}}\right)=\mathrm{H}^{i}\left(U(L)_{P}, d_{e}\right)=\mathrm{H}^{i}\left(\operatorname{Sym}(L)_{P}, d_{e}\right)=\Lambda^{i}\left(T_{\lambda_{0}}\right)$, where $T_{\lambda_{0}}$ is the tangent space to $\mathcal{C}$ at the point $\lambda_{0} \neq 0$. It follows from this that $\mathrm{H}^{i}\left(\operatorname{Sym}^{i}(L)_{P}, d_{e}\right)=\Lambda^{i}\left(T_{\lambda_{0}}\right)$ and $\mathrm{H}^{j}\left(\operatorname{Sym}^{i}(L)_{P}, d_{e}\right)=0, i \neq j$. In particular, for $i=1$ we obtain $\mathrm{H}^{1}\left(L_{P}\right)=T_{\lambda_{0}}, \mathrm{H}^{j}\left(L_{P}\right)=0$ if $j>1$.

The corollary follows from Proposition $\lcm{36}$ because $\mathcal{C}$ is a smooth homogeneous space away from $\lambda=0$.

Recall that Lie algebra $L$ as a vector space is equal to $L^{1}+Y M$. The action of the differential $d_{e}$ is $P$-covariant. This fact together with the information about the cohomology of $L_{P}$ permits us to calculate the action of $d_{e}$ on $L_{P}$ and on $Y M_{P}$.

Recall that

$$
L_{P}=L^{1} \otimes \mu_{-1}+L^{2}+L^{3} \otimes \mu_{1}+\ldots
$$

We describe the differential $d_{e}$ on $L^{1} \otimes \mu_{-1}$ using decomposition (42,43).

It follows from (42) that $L^{1} \otimes \mu_{-1}$ has $W^{*}$ as factor -representation, i.e there exists a surjective homomorphism $\phi: L^{1} \otimes \mu_{-1} \rightarrow W^{*}$. We conclude from Schur's lemma that $d_{e}$ maps $L^{1} \otimes \mu_{-1}$ onto $W^{*} \subset L^{2}$ and coincides with $\phi$ up to a constant factor. From the information about the cohomology of $L_{P}$ we infer that the constant factor does not vanish. Taking into account that the $\mathrm{H}^{i}\left(L_{P}, d_{e}\right)=0$ for $i>1$ we obtain that the complex $L^{1} / \operatorname{Ker} d_{e} \rightarrow L^{2} \rightarrow \ldots$ is acyclic. If we truncate $L^{1} / \operatorname{Ker} d_{e}$ term, the resulting complex will have cohomology equal to $d_{e}\left(L^{1} / \operatorname{Ker} d_{e}\right)=W^{*}$. This proves that the embedding $W^{*} \subset Y M_{P}$ is a quasiisomorphism.

To derive from this statement that the embedding of vector bundles $\mathcal{W}^{*} \subset$ $\mathcal{Y} \mathcal{M}$ generates isomorphism of hypercohomology we notice that this embedding induces a homomorphism of spectral sequences calculating the hypercohomology. It is easy to check that the above statement implies isomorphism of $E_{2}$ terms, hence isomorphism of hypercohomology.

From Künneth theorem we can conclude that the embedding $\operatorname{Sym}^{j} W \subset$ $\operatorname{Sym}^{j} Y M_{P}$ is a quasi- isomorphism; using spectral sequences we derive isomorphism of hypercohomology of corresponding complexes of vector bundles. 
We can give a similar analysis of the complex $\mathcal{T Y M}^{\bullet}$. Indeed we have a short exact sequence of complexes

$$
0 \rightarrow \mathcal{T Y M}^{\bullet} \rightarrow \mathcal{Y} \mathcal{M}^{\bullet} \rightarrow \mathcal{L}^{2} \rightarrow 0
$$

where $\mathcal{L}^{2}$ is a trivial vector bundle over $\mathcal{Q}$ with a fiber $L^{2}$. The short exact sequence gives rise to short exact sequence of corresponding $P$-modules and to a long exact sequence of their cohomology:

$$
\begin{aligned}
& 0 \rightarrow \mathrm{H}^{0}\left(Y M_{P}, d_{e}\right) \rightarrow L^{2} \rightarrow \mathrm{H}^{1}\left(T Y M_{P}, d_{e}\right) \rightarrow 0 \\
& \mathrm{H}^{i}\left(T Y M_{P}, d_{e}\right)=\mathrm{H}^{i}\left(Y M_{P}, d_{e}\right) \quad i \geq 2
\end{aligned}
$$

By definition $\mathrm{H}^{0}\left(T Y M_{P}, d_{e}\right)=0$. Taking into account quasiisomorphism between $W^{*}$ and $Y M_{P}$ we get an exact sequence of Spin(10)-modules

$$
0 \rightarrow W^{*} \rightarrow L^{2} \rightarrow \mathrm{H}^{1}\left(T Y M_{P}, d_{e}\right) \rightarrow 0
$$

It follows from the decomposition (43) that there is only one Spin(10)equivariant embedding of $\operatorname{Spin}(10)$-modules: $W^{*} \rightarrow L^{2}$. Also the module $L^{2} / W^{*}$ is isomorphic to $W$. From this we conclude that $\mathrm{H}^{1}\left(T Y M_{P}, d_{e}\right)$ is isomorphic to $W$. Due to isomorphisms (141) the complex $T Y M_{P}$ has no higher cohomology. We see that the embedding $W \rightarrow T Y M_{P}$ is a quasi-isomorphism. Again using spectral sequences we obtain that the embedding

$$
\mathcal{W} \rightarrow \mathcal{T Y M}^{\bullet}
$$

generates an isomorphism of hypercohomology.

\section{F Construction of the supersymmetric deforma- tions}

We will show that starting with $G^{\alpha}$ obeying

$$
\theta_{\alpha} G^{\alpha}=0
$$


on solutions of the SYM equations we can construct a SUSY deformation of SYM. Most of these deformation are given by the general formula (56). We will describe solutions that lead to exceptional deformations corresponding to (59) and (62).

First of all we can take

$$
G^{\alpha}=\chi^{\alpha}
$$

Then $\theta_{\alpha} \chi^{\alpha}=\Gamma_{\alpha}^{\alpha i j} F_{i j}=0$.

We shall denote

$$
E_{1} \circ \cdots \circ E_{n}=\frac{1}{n !} \sum_{\sigma \in S_{n}} E_{\sigma(1)} \cdots E_{\sigma(n)}
$$

the symmetrized product of operators. We shall be also using freely a generalization to the formula to the $\mathbb{Z}_{2}$-graded case. There is another interesting solution is

$$
G^{\alpha}=2 \Gamma_{\beta}^{\alpha i j k l} F_{i j} \circ F_{k l} \circ \chi^{\beta}+7 F_{i j} \circ F_{i j} \circ \chi^{\alpha}
$$

Let us verify that $G^{\alpha}$ (145) satisfies (143). We use $\circ$ for symmetrized product of operators (see Section ??). It is an immediate corollary of (5) that $\theta_{\alpha} \Gamma_{\beta}^{\alpha i j k l} F_{i j} \circ F_{k l} \circ \chi^{\beta}=-4 \Gamma_{\beta}^{\alpha i j k l} \Gamma_{\alpha \gamma}^{i} \nabla_{j} \chi^{\gamma} \circ F_{k l} \circ \chi^{\beta}+\Gamma_{\beta}^{\alpha i j k l} \Gamma_{\alpha}^{\beta s t} F_{i j} \circ F_{k l} \circ F_{s t}$ Let $T^{i j k l s t} F_{i j} \circ F_{k l} \circ F_{s t}$ be equal to $\Gamma_{\beta}^{\alpha i j k l} \Gamma_{\alpha}^{\beta s t} F_{i j} \circ F_{k l} \circ F_{s t}$. The tensor $T^{i j k l s t}$ is obtained from $\Gamma_{\beta}^{\alpha i j k l} \Gamma_{\alpha}^{\beta s t}$ by symmetrization in $(i j)(k l)(s t)$ groups of indices. $T^{i j k l s t}$ is zero because it defines an $\mathfrak{s o}_{10}$-invariant polynomial function of degree three on the adjoint representation of $\mathfrak{s o}_{10}$. It is well known (43]) that such invariants for Lie algebras of $\mathfrak{s o - t y p e ~ e x i s t ~ o n l y ~ i n ~ d e g r e e s ~ d i v i s i b l e ~ b y ~ f o u r . ~}$

We omit verification of

$$
\Gamma_{\beta}^{\alpha i j k l} \Gamma_{\alpha \gamma}^{i}=\frac{14}{4} \Gamma_{\beta \gamma}^{j k l}=\frac{14}{4}\left(\Gamma_{\beta \delta}^{j} \Gamma^{\delta \epsilon k} \Gamma_{\epsilon \gamma}^{l}-\frac{1}{2} \delta^{j k} \Gamma_{\beta \gamma}^{l}-\frac{1}{2} \delta^{k l} \Gamma_{\beta \gamma}^{j}+\frac{1}{2} \delta^{j l} \Gamma_{\beta \gamma}^{k}\right)
$$

$\Gamma$-matrix $\Gamma_{\beta \gamma}^{j k l}$ is skew-symmetric in $\beta \gamma$. We infer that

$$
\begin{aligned}
& \theta_{\alpha} \Gamma_{\beta}^{\alpha i j k l} F_{i j} \circ F_{k l} \circ \chi^{\beta}=14\left(\Gamma_{\beta \delta}^{j} \Gamma^{\delta \epsilon k} \Gamma_{\epsilon \gamma}^{l}-\frac{1}{2} \delta^{j k} \Gamma_{\beta \gamma}^{l}-\frac{1}{2} \delta^{k l} \Gamma_{\beta \gamma}^{j}+\frac{1}{2} \delta^{j l} \Gamma_{\beta \gamma}^{k}\right) \nabla_{j} \chi^{\beta} \circ F_{k l} \circ \chi^{\gamma}= \\
& =-14 \Gamma_{\beta \gamma}^{l} \nabla_{j} \chi^{\beta} \circ F_{j l} \circ \chi^{\gamma}
\end{aligned}
$$


The term that contains $\Gamma_{\beta \delta}^{j} \Gamma^{\delta \epsilon k} \Gamma_{\epsilon \gamma}^{l}$ vanishes by virtue of the Dirac equation. The term with $\frac{1}{2} \delta^{k l} \Gamma_{\beta \gamma}^{j}$ disappears because $\delta^{k l} F_{k l}=0$. If we compare

$$
\theta_{\alpha} F_{i j} \circ F_{i j} \circ \chi^{\alpha}=4 \Gamma_{\beta \alpha}^{j} \nabla_{i} \chi^{\beta} \circ F_{i j} \circ \chi^{\alpha}
$$

with (146) we conclude that $G^{\alpha}$ (145) satisfies (143).

The space of solutions of (143) contains a set of trivial solutions $G^{\alpha}=\theta_{\beta} G^{\alpha \beta}$ with $\Gamma$-traceless $G^{\alpha \beta}$. Indeed by virtue of (7)

$$
\theta_{\alpha} G^{\alpha}=\Gamma_{\alpha \beta}^{i} D_{i} G^{\alpha \beta}=0
$$

It is natural to identify solutions of (143), that differ by a trivial solution.

Our interest to the group $H$ of equivalence classes of such solutions has been stimulated by existence of a map from $H$ to infinitesimal supersymmetric deformations of the equations of motion of SYM

$$
\frac{\delta \mathcal{L}_{S Y M}}{\delta A_{i}}+\epsilon \mathcal{M}_{i} \quad \frac{\delta \mathcal{L}_{S Y M}}{\delta \chi^{\alpha}}+\epsilon \mathcal{M}_{\alpha}
$$

We omit verification that infinitesimal field redefinition $\mathcal{N}^{i}, \mathcal{N}^{\alpha}$ transforms

$$
\begin{aligned}
& \mathcal{M}_{i} \rightarrow \mathcal{M}_{i}+D_{i} D_{j} \mathcal{N}_{\gamma j}-2 D_{j} D_{i} \mathcal{N}_{\gamma j}+D_{j} D_{j} \mathcal{N}_{\gamma i}+\Gamma_{\alpha \beta}^{i}\left[\chi^{\alpha}, \mathcal{N}_{\gamma}^{\beta}\right] \\
& \mathcal{M}_{\alpha} \rightarrow \mathcal{M}_{\alpha}-\Gamma_{\alpha \beta}^{j}\left[\chi^{\beta}, \mathcal{N}_{\gamma j}\right]-\Gamma_{\alpha \beta}^{i} D_{i} \mathcal{N}_{\gamma}^{\beta}
\end{aligned}
$$

The following map generalizes the operator $A$ (57):

$$
\mathcal{M}_{i}=I_{\alpha}^{i} G^{\alpha} \quad \mathcal{M}_{\alpha}=I_{\alpha \beta} G^{\beta}
$$

with $I_{\alpha}^{i}\left(\theta_{1}, \ldots, \theta_{16}\right)$ and $I_{\alpha \beta}\left(\theta_{1}, \ldots, \theta_{16}\right)$ being some ingeniously chosen noncommutative polynomials in supersymmetry operators. Operators $I_{\alpha}^{i}, I_{\alpha \beta}$ satisfy certain equations that ensure supersymmetry of (147).

Operators $I, I_{\alpha}^{i}$, and $I_{\alpha \beta}$ satisfy an analogue of the descent equation (see e.g. [45]). To simplify notations we shall work with functions

$$
I^{i}(\lambda)=I_{\alpha}^{i} \lambda^{\alpha}, \quad I_{\alpha}(\lambda)=I_{\alpha \beta} \lambda^{\beta}, \quad \theta(\lambda)=\theta_{\alpha} \lambda^{\alpha} \ldots
$$

in pure spinor variables $\lambda^{\alpha}$. There are several equations they satisfy (see Appendix (G). The most relevant to our present needs are

$$
I \theta(\lambda)=D_{i} I^{i}(\lambda)+\chi^{\beta} I_{\beta}(\lambda)
$$




$$
\begin{aligned}
& \theta_{\gamma} I=D_{i} B_{\gamma}^{i}+\chi^{\beta} B_{\gamma, \beta} \\
& \theta_{\gamma}^{R_{1}}\left(\begin{array}{c}
I^{i}(\lambda) \\
I_{\beta}(\lambda)
\end{array}\right)=\left(\begin{array}{c}
B_{\gamma}^{i} \theta(\lambda) \\
B_{\gamma, \beta} \theta(\lambda)
\end{array}\right)+d_{1}\left(\begin{array}{c}
C_{\gamma}^{i}(\lambda) \\
C_{\gamma}^{\delta}(\lambda)
\end{array}\right)
\end{aligned}
$$

Operators $B, C$ likewise $I$ are noncommutative polynomials in $\theta_{\alpha}$. The reader should consult formulas (160[161) and (158) for the action of the supersymmetry $\theta_{\alpha}^{R_{i}} i=1,2$ and the operator $d_{1}$. We refer the reader to Appendix $\mathrm{G}$ for the details. Let us introduce an infinitesimal field redefinition

$$
\mathcal{N}_{\gamma i}=C_{\gamma \beta, i} G^{\beta} \quad \mathcal{N}_{\gamma}^{\alpha}=C_{\gamma \beta}^{\alpha} G^{\beta}
$$

with $\beta$ a pure spinor index.

The immediate corollary of (149) is that $\mathcal{M}_{i} \mathcal{M}_{\alpha}$ in (147) satisfy (157) for any $G^{\alpha}$, that solves (143):

$$
\nabla_{i} \mathcal{M}_{i}+\left[\chi^{\beta}, \mathcal{M}_{\alpha}\right]=D_{i} I_{\alpha}^{i} G^{\alpha}+\chi^{\alpha} I_{\alpha \beta} G^{\beta}=I \theta_{\alpha} G^{\alpha}=0
$$

Supersymmetry of this deformation follows from (150):

$$
\begin{aligned}
& \theta_{\gamma} \mathcal{M}_{i}+2 \Gamma_{\gamma}^{\beta i j} D_{j} \mathcal{M}_{\beta}=D_{i} D_{j} \mathcal{N}_{\gamma j}-2 D_{j} D_{i} \mathcal{N}_{\gamma j}+D_{j} D_{j} \mathcal{N}_{\gamma i}+\Gamma_{\alpha \beta}^{i}\left[\chi^{\alpha}, \mathcal{N}_{\gamma}^{\beta}\right] \\
& -\theta_{\gamma} \mathcal{M}_{\beta}+\Gamma_{\gamma \beta}^{i} \mathcal{M}_{i}=-\Gamma_{\alpha \beta}^{j}\left[\chi^{\beta}, \mathcal{N}_{\gamma j}\right]-\Gamma_{\alpha \beta}^{i} D_{i} \mathcal{N}_{\gamma}^{\beta}
\end{aligned}
$$

Note that the terms involving $B_{\gamma}^{i} \theta_{\beta} G^{\beta}, B_{\gamma \alpha} \theta_{\beta} G^{\beta}$ drop out because $G^{\beta}$ satisfies (143).

\section{G A deformation complex}

The goal of this Appendix is to interpret equations (149) and (150) as a part of a more general system of equations. A solution of this system is a cocycle in a certain bi-complex:

$$
\begin{array}{lllllll}
\ldots & & \ldots & & \cdots & & \ldots \\
\uparrow d_{1}^{I I} & & \uparrow d_{1}^{I I} & & \uparrow d_{1}^{I I} & & \uparrow d_{1}^{I I} \\
E_{0}^{1} & \stackrel{d_{0}^{I}}{\leftarrow} & E_{1}^{1} & \stackrel{d_{1}^{I}}{\leftarrow} & E_{2}^{1} & \stackrel{d_{2}^{I}}{\leftarrow} & E_{3}^{1} \\
\uparrow d_{0}^{I I} & & \uparrow d_{0}^{I I} & & \uparrow d_{0}^{I I} & & \uparrow d_{0}^{I I} \\
E_{0}^{0} & \stackrel{d_{0}^{I}}{\leftarrow} & E_{1}^{0} & \stackrel{d_{1}^{I}}{\leftarrow} & E_{2}^{0} & \stackrel{d_{2}^{I}}{\leftarrow} & E_{3}^{0}
\end{array}
$$


The horizontal differential $d^{I}$ is defines a four-term complex

$$
R_{0} \stackrel{d_{0}}{\leftarrow} R_{1} \stackrel{d_{1}}{\leftarrow} R_{2} \stackrel{d_{2}}{\leftarrow} R_{3}
$$

In the simplest form the linear spaces $R_{0}, R_{1}, R_{2}, R_{3}$ of the complex are formed by noncommutative polynomials with constant coefficients in covariant derivatives of curvature and spinor field with values in sections of adjoint bundle , the fields satisfy equations of motion of SYM. The complex governs infinitesimal deformations of SYM. The space $R_{1}$ contains infinitesimal deformations $\left(\mathcal{M}_{i}(A, \chi), \mathcal{M}_{\alpha}(A, \chi)\right)$ of Euler-Lagrange equation

$$
\frac{\delta \mathcal{L}_{S Y M}}{\delta A_{i}}+h \mathcal{M}_{i}=0 \quad \frac{\delta \mathcal{L}_{S Y M}}{\delta \chi^{\alpha}}+h \mathcal{M}^{\alpha}
$$

The space $R_{2}$ contains infinitesimal field redefinitions $\left(\mathcal{N}_{i}(A, \chi), \mathcal{N}^{\alpha}(A, \chi)\right)$ :

$$
A_{i} \rightarrow A_{i}+h \mathcal{N}_{i} \quad \chi^{\alpha} \rightarrow \chi^{\alpha}+h \mathcal{N}^{\alpha}
$$

The spaces $R_{0}, R_{3}$ are spanned by $\mathcal{Y}(A, \chi)$, which, as we already mentioned, in case $R_{0}$ define a Lagrangian density $\operatorname{tr} \mathcal{Y}(A, \chi)$. A cohomology of $d_{2}$ we interpret as an infinitesimal automorphism of a solution $\left(\nabla_{i}, \chi^{\alpha}\right)$.

The differential $d_{0}, d_{1}, d_{2}$ have forms

$$
d_{0}\left(\begin{array}{c}
\mathcal{M}_{i} \\
\mathcal{M}_{\alpha}
\end{array}\right)=D_{i} \mathcal{M}_{i}+\left[\chi^{\alpha}, \mathcal{M}_{\alpha}\right]
$$

$d_{0}$ can be obtained from

$$
\nabla_{i} \frac{\delta \mathcal{L}}{\delta A_{i}}+\left[\chi^{\alpha} \frac{\delta \mathcal{L}}{\delta \chi^{\alpha}}\right]=0
$$

by variation (154).

$$
d_{1}\left(\begin{array}{c}
\mathcal{N}_{i} \\
\mathcal{N}^{\alpha}
\end{array}\right)=\left(\begin{array}{c}
D_{i} D_{j} \mathcal{N}_{j}-2 D_{j} D_{i} \mathcal{N}_{j}+D_{j} D_{j} \mathcal{N}_{i}+\Gamma_{\alpha \beta}^{i}\left[\chi^{\alpha}, \mathcal{N}^{\beta}\right] \\
-\Gamma_{\alpha \beta}^{j}\left[\chi^{\beta}, \mathcal{N}_{j}\right]-\Gamma_{\alpha \beta}^{j} D_{j} \mathcal{N}^{\beta}
\end{array}\right)
$$

$d_{1}\left(\begin{array}{c}\mathcal{N}_{i} \\ \mathcal{N}^{\alpha}\end{array}\right)$ is a variation of equations (34) under infinitesimal field redefinition (155).

$$
d_{2} \mathcal{Y}=\left(\begin{array}{c}
D_{j} \mathcal{Y} \\
-\left[\chi^{\alpha}, \mathcal{Y}\right]
\end{array}\right)
$$


$d_{2} \mathcal{Y}$ stands for a field-dependent infinitesimal gauge transformation. For the first time the non-supersymmetric analogue of the complex $R$ has been analyzed in mathematical literature in [18. The variant that contains fermions and scalar bosons has been worked out in [35]. The differentials $d_{i}$ commute with supersymmetries

$$
\begin{gathered}
\theta_{\alpha}^{R_{i}}(\mathcal{Y})=\theta_{\alpha} \mathcal{Y} \quad i=0,3 \\
\theta_{\alpha}^{R_{1}}\left(\begin{array}{c}
\mathcal{M}_{i} \\
\mathcal{M}_{\alpha}
\end{array}\right)=\left(\begin{array}{c}
\theta_{\alpha} \mathcal{M}_{i}+2 \Gamma_{\alpha}^{\beta i j} D_{j} \mathcal{M}_{\beta} \\
-\theta_{\alpha} \mathcal{M}_{\beta}+\Gamma_{\alpha \beta}^{i} \mathcal{M}_{i}
\end{array}\right) \\
\theta_{\alpha}^{R_{2}}\left(\begin{array}{c}
\mathcal{N}_{i} \\
\mathcal{N}^{\beta}
\end{array}\right)=\left(\begin{array}{c}
\theta_{\alpha} \mathcal{N}_{i}+\Gamma_{\alpha \gamma}^{i} \mathcal{N}^{\gamma} \\
-\theta_{\alpha} \mathcal{N}^{\beta}-2 \Gamma_{\alpha}^{\beta i j} D_{i} \mathcal{N}_{j}
\end{array}\right)
\end{gathered}
$$

We do not give here the formulas for infinitesimal shift operators in $R_{i}$, but mention in passing that they act trivially in cohomology as operators $\mathcal{Y}, \mathcal{M}, \mathcal{N}$ have space-time constant coefficients. The algebra generated by operators $\theta_{\alpha}^{R_{i}}, i=$ 1,2 is rather complex. However operators $\theta_{\alpha}^{R_{i}}, i=0,1,2,3$ anti-commute in cohomology.

In a more sophisticated version $\Theta$ of the complex $R$, which is used in construction of the bicomplex (153), $\mathcal{Y}, \mathcal{M}_{i}, \mathcal{M}_{\alpha}, \mathcal{N}_{i}, \mathcal{N}^{\alpha}$ are noncommutative polynomials in $\theta_{\alpha}$. Operators $d_{0}, d_{1}, d_{2}$ can be adapted to $\Theta$ if we replace $D_{i}$ and $\left[\chi^{\alpha}, \cdot\right]$ in the formulas $(156[158 \mid 159)$ by

$$
\begin{gathered}
D_{i}=1 / 8 \Gamma_{i}^{\alpha \beta} \theta_{\alpha} \theta_{\beta} . \\
1 / 5 \Gamma_{i}^{\delta \gamma}\left[\theta_{\beta}, D_{i}\right]=1 / 40 \Gamma_{i}^{\delta \gamma} \Gamma_{i}^{\alpha \beta}\left[\theta_{\gamma}, \theta_{\alpha} \theta_{\beta}\right] .
\end{gathered}
$$

The bicomplex $E=\bigoplus_{i j} E_{i}^{j}$ is the tensor product

$$
E_{i}^{j}=\Theta_{i} \otimes \mathcal{S}_{j}
$$

The differential $d^{I I}$ is a right multiplication on $\pm \theta(\lambda)= \pm \theta_{\alpha} \lambda^{\alpha}$ :

$$
\mathcal{Y}, \mathcal{M}_{i}, \mathcal{M}_{\alpha}, \mathcal{N}_{i}, \mathcal{N}^{\alpha} \rightarrow \mathcal{Y} \theta(\lambda), \mathcal{M}_{i} \theta(\lambda), \mathcal{M}_{\alpha} \theta(\lambda), \mathcal{N}_{i} \theta(\lambda), \mathcal{N}^{\alpha} \theta(\lambda)
$$


The operator $c_{0}=I$ (57) is an element of $E_{0}^{0}$, operators $c_{1}=\left(I^{i}(\lambda), I_{\alpha}(\lambda)\right)$ (148) belong to $E_{1}^{1}$. There are also $c_{2}=\left(\widetilde{I}^{i}(\lambda), \widetilde{I}^{\alpha}(\lambda)\right)$ and $c_{3}=\widetilde{I}(\lambda)$-functions of degree two and three in $\lambda$. The full set of equations on $c=\left(c_{0}, c_{1}, c_{2}, c_{3}\right)$ that generalizes (149) is:

$$
d_{0}^{I I} c_{0}=d_{0}^{I} c_{1} \quad d_{1}^{I I} c_{1}=d_{0}^{I} c_{2} \quad d_{2}^{I I} c_{2}=d_{0}^{I} c_{3} \quad d_{3}^{I I} c_{3}=0
$$

The cohomology of the diagonal complex $\operatorname{Tot}(E)^{k}=\bigoplus_{k=j-i} E_{i}^{j}$ (153) is intractable, but if we choose to work with all the gauge groups $\mathrm{U}(N)$ simultaneously, i.e. let $\mathcal{Y}, \mathcal{M}_{i}, \mathcal{M}_{\alpha}, \mathcal{N}_{i}, \mathcal{N}^{\alpha}$ be noncommutative polynomials in $\boldsymbol{\theta}_{\alpha} \in L$ (see Section 2.1) then cohomology are finite dimensional:

$H^{0}(\operatorname{Tot}(E))=H^{3}(\operatorname{Tot}(E))=\mathbb{C} \quad \mathrm{H}^{1}(\operatorname{Tot}(E))=V^{*}+S^{*} \quad \mathrm{H}^{2}(\operatorname{Tot}(E))=V+S$

Collection $c_{0}, c_{1}, c_{2}, c_{3}$ is a generator in $\mathrm{H}^{0}(\operatorname{Tot}(E))$. Note that $c_{3}$ satisfies $d_{3}^{I I} c_{3}=0$. In fact it is a generator of the only nontrivial $\theta(\lambda)$-cohomology class in $U(L) \otimes \mathcal{S}$. It contains a representative that can be conveniently written in terms of graded symmetric product and differentiations (25):

$$
\Gamma_{\alpha \beta}^{m} \lambda^{\alpha} \Gamma_{\gamma \delta}^{n} \lambda^{\gamma} \Gamma_{\epsilon \varepsilon}^{k} \lambda^{\epsilon} \Gamma_{\mu \nu}^{m n k} \frac{\partial^{5}}{\partial \theta_{\beta} \partial \theta_{\delta} \partial \theta_{\varepsilon} \partial \theta_{\mu} \partial \theta_{\nu}} \theta_{1} \circ \cdots \circ \theta_{16}
$$

To carry out the computations of $c_{1}$ we choose $\theta_{\alpha}$ to be a weight basis. Then $\Gamma_{\alpha \alpha}^{i}=0$. Operators $\left(\begin{array}{c}I^{i}(\lambda) \\ I_{\beta}(\lambda)\end{array}\right)$ (147/149) can be computed by the formula:

$$
\begin{aligned}
& \left(\begin{array}{l}
I^{i}(\lambda) \\
I_{\beta}(\lambda)
\end{array}\right)=\sum_{\beta<\alpha_{1}} \sum_{k>0} \\
& \left(\sum_{\alpha_{2 k+1}<\cdots<\alpha_{1}}(-1)^{\alpha_{1}+\alpha_{3}+\cdots+\alpha_{2 k+1}}\left(\theta_{\alpha_{2 k+1}}^{R_{1}} \cdots \theta_{\alpha_{2}}^{R_{1}}\left(\underset{\left.\theta_{\alpha_{1}}, \theta_{\beta}\right] \lambda^{\beta}}{0}\right)\right) \theta_{1} \cdots \widehat{\theta}_{\alpha_{2 k+1}} \cdots \widehat{\theta}_{\alpha_{1}} \cdots \theta_{16}+\right. \\
& \left.+\sum_{\alpha_{2 k}<\cdots<\alpha_{1}}(-1)^{\alpha_{1}+\alpha_{3}+\cdots+\alpha_{2 k-1}+\alpha_{2 k}+1}\left(\theta_{\alpha_{2 k}}^{R_{1}} \cdots \theta_{\alpha_{2}}^{R_{1}}\left(\underset{\left[\theta_{\alpha_{1}}, \theta_{\beta}\right] \lambda^{\beta}}{0}\right)\right) \theta_{1} \cdots \widehat{\theta}_{\alpha_{2 k}} \cdots \widehat{\theta}_{\alpha_{1}} \cdots \theta_{16}\right)
\end{aligned}
$$

The summands in the formula should be understood as follows. We apply consequently operators $\theta_{\alpha_{i}}^{R_{1}}(160)$ to $\left(\begin{array}{c}{\left[\theta_{\alpha_{1}}, \theta_{\beta}\right] \lambda^{\beta}} \\ 0\end{array}\right)$. Then we multiply from 
the right components of the resulting two-vector on $\theta_{1} \cdots \widehat{\theta}_{\alpha_{n}} \cdots \widehat{\theta}_{\alpha_{1}} \cdots \theta_{16}$ The proof is based on the formula

$$
\begin{aligned}
& \theta_{1} \cdots \theta_{16} \theta_{\beta}=\sum_{\beta<\alpha_{1}} \sum_{k=1}^{n} \\
& \left(\sum _ { \alpha _ { 2 k + 1 } < \cdots < \alpha _ { 1 } } ( - 1 ) ^ { \alpha _ { 1 } + \alpha _ { 3 } + \cdots + \alpha _ { 2 k + 1 } } \left[\theta_{\alpha_{2 k+1}},\left[\ldots,\left[\theta_{\alpha_{1}}, \theta_{\beta}\right]\right] \theta_{1} \cdots \widehat{\theta}_{\alpha_{2 k+1}} \cdots \widehat{\theta}_{\alpha_{1}} \cdots \theta_{16}+\right.\right. \\
& \sum_{\alpha_{2 k}<\cdots<\alpha_{1}}(-1)^{\alpha_{1}+\alpha_{3}+\cdots+\alpha_{2 k-1}+\alpha_{2 k}+1}\left[\theta_{\alpha_{2 k}},\left[\ldots,\left[\theta_{\alpha_{1}}, \theta_{\beta}\right]\right] \theta_{1} \cdots \widehat{\theta}_{\alpha_{2 k}} \cdots \widehat{\theta}_{\alpha_{1}} \cdots \theta_{16}\right) \\
& +r_{n}
\end{aligned}
$$

where

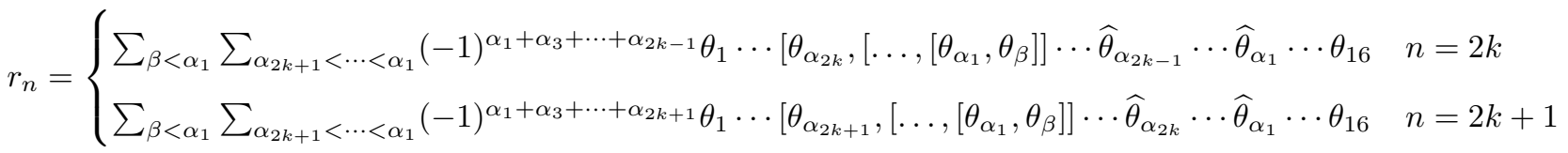

It can verified by induction on $n$.

Few words about the proof of existence of $c$. The algebra $U(L)$ is a free left $U(Y M)$-module. Then $0=\mathrm{H}_{i}(Y M, U(L))=\mathrm{H}^{3-i}(Y M, U(L))$ for $i \geq 1$. The complex $\Theta$ computes $\mathrm{H}^{i}(Y M, U(L))$. The element $d^{I I} c_{1}$ is a $d^{I}$-cocycle. By the vanishing result the cocyle $d^{I I} c_{1}$ is $d^{I}$-trivial: $d^{I I} c_{1}=d^{I} c_{2}$. We find $c_{3}$ along the same lines. We leave the proof of non-triviality of $c$ to the reader.

Note that $\theta_{\alpha} c=\left(\theta_{\alpha}^{R_{0}} c_{0}, \theta_{\alpha}^{R_{1}} c_{1}, \theta_{\alpha}^{R_{2}} c_{2}, \theta_{\alpha}^{R_{3}} c_{3}\right)$ is a cocycle (the action of the supersymmetries commutes with the differential). $\theta_{\alpha} c$ has a degree in $\theta$ on one greater then $c$. This means that $\theta_{\alpha} c$ is a trivial cocycle. The second equation in (150) follows from this.

\section{H Dimensional reductions of SYM}

We have analyzed supersymmetric deformations of ten-dimensional SYM theory and of this theory reduced to a point. Similar methods can be applied to $d$ dimensional reduction of ten-dimensional SYM theory for any $d \leq 10$. Here we discuss some general results in this direction leaving concrete calculations for the future work. 
We can generalize the results of Section 4 introducing the Lie algebra $T_{d} Y M$. Recall that the Lie algebra $Y M$ as a vector space can be represented as a direct sum $\sum_{n \geq 2} L^{n}$ where $L^{2}$ has a basis $\boldsymbol{D}_{1}, \ldots, \boldsymbol{D}_{10}$. We define $T_{d} Y M$ as a subalgebra $\left(L^{2}\right)^{\prime}+\sum_{n \geq 3} L^{n}$ of the algebra $Y M$. Here $\left(L^{2}\right)^{\prime}$ is spanned by $\boldsymbol{D}_{d+1}, \ldots, \boldsymbol{D}_{10}$. (In more invariant way one can say that $\left(L^{2}\right)^{\prime}$ is a $(10-d)$ dimensional subspace of $L^{2}$, such that the restriction of the inner-product from $L^{2}$ to $\left(L^{2}\right)^{\prime}$ is non-degenerate.) We will modify below the arguments of Appendix $\mathrm{C}$ to obtain the following generalization of theorems 11 and 12 :

Proposition 38 Every element of $\mathrm{H}^{\bullet}\left(L, U\left(T_{d} Y M\right)\right)$ where $k \geq 2$ specifies an infinitesimal supersymmetric deformation of equations of motion of SYM theory reduced to d-dimensional space.

This statement should be understood in the framework of BV-formalism. In this formalism we interpret invariance with respect to Lie algebra $\mathfrak{g}$ in terms of $\mathrm{L}_{\infty}$ action (Section 6 and Appendix A). The solutions of the equations of motion correspond to the points of zero locus of the vector field $Q$; the invariance of equations of motion with respect to $\mathrm{L}_{\infty}$ action is expressed by the equation

$$
d_{\mathfrak{g}} q+[Q, q]+\frac{1}{2}[q, q]=0 .
$$

where $q$ is a ghost dependent vector field. Infinitesimal deformations of $Q$ and $q$ obeying this equation are labeled by elements of some homology group that can be interpreted as Lie-Hochschild cohomology $\operatorname{HH}_{\mathfrak{g}}^{\bullet}(\mathcal{A}, \mathcal{A})$; in our case $\mathfrak{g}=\mathfrak{s u s y}_{d}$ stands for the algebra of supersymmetries in dimension 10 reduced to the dimension $d$ and for $\mathcal{A}$ we can take the algebra $b v_{d}$ obtained from $b v$ by means of dimensional reduction. 38 We can use pure spinor formalism also in the case of reduction to $d$ dimensions; then for $\mathcal{A}$ we should take $d$-dimensional reduction of Berkovits algebra $B$ that can be defined as the algebra $B_{d}$ of polynomial functions depending of pure spinor $\lambda$, odd spinor $\psi$ and $x \in \mathbb{R}^{d}$ with the differential

${ }^{38}$ Notice, that the most interesting deformations correspond to the elements of $\operatorname{HH}_{\mathfrak{g}}^{k}(\mathcal{A}, \mathcal{A})$ with $k=2$; the elements with $k>2$ correspond to deformation of higher terms in $\mathrm{L}_{\infty}$ action; these higher terms do not have direct physical meaning. 
defined as the derivation

$$
\lambda^{\alpha}\left(\frac{\partial}{\partial \psi^{\alpha}}+\Gamma_{\alpha \beta}^{i} \psi^{\beta} \frac{\partial}{\partial x^{i}}\right) .
$$

The algebra $B_{d}$ is a supercommutative quadratic algebra; its Koszul dual is a universal enveloping algebra of differential graded Lie algebra $\tilde{L}_{d}$ defined as an algebra with generators $\boldsymbol{\theta}_{1}, \ldots, \boldsymbol{\theta}_{16}$ of degree one, generators $s_{1}, \ldots, s_{16}$ of degree zero,and generators $\varsigma_{1}, \ldots, \varsigma_{d}$ of degree 1 . They satisfy relations

$$
\begin{gathered}
\Gamma_{i_{1}, \ldots, i_{5}}^{\alpha \beta}\left[\boldsymbol{\theta}_{\alpha}, \boldsymbol{\theta}_{\beta}\right]=0 . \\
{\left[\boldsymbol{\theta}_{\alpha}, s_{\beta}\right]=\Gamma_{\alpha \beta}^{i} \varsigma_{i}}
\end{gathered}
$$

(Other commutation relations are trivial.) The differential acts by the formulas $d\left(\boldsymbol{\theta}_{\alpha}\right)=s_{\alpha}, d\left(\boldsymbol{D}_{i}\right)=\varsigma_{i}$ for $i \leq d, d\left(\boldsymbol{D}_{i}\right)=0$ for $i>d$. It is easy to construct a natural embedding of $T_{d} Y M$ into $\tilde{L}_{d}$ and to prove that this embedding is a quasi-isomorphism; similar statements are true for their universal envelopes. These statements together with the fact that $B_{d}$ is a Koszul algebra permit us to say that $B_{d}$ is dual to $U\left(T_{d} Y M\right)$ in the sense of Appendix B. From other side, $U\left(T_{d} Y M\right)$ is dual to $C^{\bullet}\left(T_{d} Y M\right)$. (Recall that $C^{\bullet}(\mathcal{G})$ denotes the algebra of polynomial functions of ghosts of Lie algebra $\mathcal{G}$ with the differential calculating the cohomology $\mathrm{H}^{\bullet}(\mathcal{G}, \mathbb{C})$; the duality between $C^{\bullet}(\mathcal{G})$ and $U(\mathcal{G})$ follows the isomorphism between Lie algebra cohomology $\mathrm{H}^{\bullet}(\mathcal{G}, \mathbb{C})$ and Hochschild cohomology $\mathrm{HH}^{\bullet}(U(\mathcal{G}), \mathbb{C})$.) We obtain that $B_{d}$ is quasi-isomorphic to $C^{\bullet}\left(T_{d} Y M\right)$, hence the supersymmetric deformations are governed by Lie-Hochschild cohomology $\mathrm{HH}_{\mathfrak{s u s y}_{d}} \bullet\left(C^{\bullet}\left(T_{d} Y M\right), C^{\bullet}\left(T_{d} Y M\right)\right)$ that can be regarded as cohomology of a complex

$$
\left.C^{\bullet}(\mathfrak{s u s y})_{d}\right) \otimes \operatorname{Sym}\left(\Pi T_{d} Y M\right)^{*} \otimes \operatorname{Sym}_{d} Y M
$$

with appropriate differential. To finish the proof we notice that this cohomology is isomorphic to $\mathrm{H}^{\bullet}\left(L, U\left(T_{d} Y M\right)\right.$. (This fact can be derived from the remark that $\left.C^{\bullet}(\mathfrak{s u s y})_{d}\right) \otimes \operatorname{Sym}\left(\Pi T_{d} Y M\right)^{*}=\mathbb{C}\left[\left[t^{1}, \ldots, t^{16}\right]\right] \otimes \operatorname{Sym}\left(\Pi Y M^{*}\right)=$ $\operatorname{Sym}\left(\Pi L^{*}\right)$.)

In Section 2.3 we have constructed vector bundles $\mathcal{W}^{*}$ and $\mathcal{W}$ over $\mathcal{Q}$. The bundle $\mathcal{W}^{*}$ is embedded into trivial vector bundle $\mathcal{V}$ with the fiber $V=L^{2}$; the 
bundle $\mathcal{W}$ is defined as a quotient $\mathcal{V} / \mathcal{W}^{*}$. We define a two-step complex of vector bundles

$$
\mathcal{V} \stackrel{p}{\rightarrow} \mathcal{W}
$$

This complex is quasi-isomorphically embedded into the complex of vector bundles $\mathcal{Y} \mathcal{M}$. This follows from the remark that it is quasi-isomorphic to the bundle $\mathcal{W}^{*}$ and from the considerations of Appendix E. We can consider a more general two-step complex

$$
\mathcal{V}^{\prime} \stackrel{p}{\rightarrow} \mathcal{W}
$$

where $\mathcal{V}^{\prime}$ is a trivial vector bundle with the fiber $V^{\prime}=\left(L^{2}\right)^{\prime}$. It is embedded into $\mathcal{T}_{d} \mathcal{Y} \mathcal{M}$ and this embedding is a quasi-isomorphism. This can be seen as follows. The zero component of the complex $\mathcal{T}_{d} \mathcal{Y} \mathcal{M}$ coincides with $V^{\prime}$. The zerotruncated complex, i.e. the complex with removed first component, coincides with $\mathcal{T Y \mathcal { M }}$. We know (see ( 142) $)$ that it is quasi-isomorphic to $\mathcal{W}$. The statement we need follows from this fact.

Observe that if we work with the unreduced theory then $V^{\prime}=0$ and the complex becomes simply the vector bundle $\mathcal{W}$ in agreement with Proposition 20. In the opposite extremity we can study the theory reduced to a point. Then $V^{\prime}=V$ and we obtain the complex (169) which is quasiisomorphic to $\mathcal{W}^{*}$. Again this agrees with the results of Proposition 14

We see that in the case at hand we can write down an analog of (72) and apply it to the calculation of $\mathrm{H}^{i}\left(L, U\left(T_{d} Y M\right)\right)$. The main remaining problem is the calculation of hypercohomology

$$
\mathbb{H}^{i}\left(\operatorname{Sym}^{k}\left(\mathcal{E}^{\bullet}\right)(j)\right)
$$

We are planning to solve this problem using the techniques of [21].

Notice that the calculation of Euler characteristic of the cohomology we consider can be done by means of methods used in Section 4 . 


\section{Representation rings}

In the set of equivalence classes of representations of a group $G$ or of $\mathfrak{g}$-modules (of representations of Lie algebra $\mathfrak{g}$ ) we can introduce a notion of sum ( direct sum) and of product (tensor product). One can consider also virtual modules (virtual representations) defined as formal differences of modules (representations). The set of equivalence classes of virtual modules can be considered as a ring with respect to direct sum and tensor product; this ring is called representation ring of group $G$ or of Lie algebra $\mathfrak{g}$. 39 If $G$ is a simply connected Lie group and $\mathfrak{g}$ denotes its Lie algebra the representation group of $G$ is isomorphic to the representation ring of $\mathfrak{g}$.

One can use another definition of representation ring that is based on consideration of representations in superpaces instead of virtual representations. (If $V$ is a module and $\Pi V$ is obtained from $V$ by means of parity reversion we assume that $V+\Pi V$ is equivalent to zero. In other words the virtual representation $V-W$ can be considered as a representation $V+\Pi W$.)

To every representation $\rho$ of a group $G$ we can assign its character $\chi$ as a function on the set of conjugacy classes of $G$ defined by the formula $\chi(g)=\operatorname{Tr} \rho(g)$. It is easy to check that the character of direct sum of representations is a sum of characters and the character of tensor product is a product of characters. Using this remark one can verify that for a compact group $G$ the representation ring is isomorphic to a subring of class functions (functions on the set of conjugacy classes of $G$.) Equivalently one can say that the representation ring is isomorphic to a subring of the ring of $W(G)$-invariant functions on maximal torus where $W(G)$ stands for the Weyl group.

A graded representation $V=\sum V_{k}$ can be considered as a power series $\sum V_{k} t^{k}$ taking values in the representation ring,

One can define some natural operations on the representation ring (the word "natural" means here that these operations are compatible with homo-

\footnotetext{
${ }^{39}$ Notice that in the definition of representation ring one can consider only finite-dimensional representations or only unitary representation, etc
} 
morphisms).

First of all we can define operations $\lambda^{i}$ sending a module $V$ into its exterior power $\Lambda^{i} V$ and operators $\sigma^{i}$ sending a module into its symmetric power. We understand here the exterior power and the symmetric power in the sense of superalgebra, hence

$$
\lambda^{i}(-V)=(-1)^{i} \sigma^{i}(V)
$$

(changing parity we exchange symmetry with antisymmetry). It is convenient to consider generating functions

$$
\begin{aligned}
& \lambda_{t}(V)=\sum \lambda^{i}(V) t^{i}, \\
& \sigma_{t}(V)=\sum \sigma^{i}(V) t^{i} .
\end{aligned}
$$

(These functions arise naturally from graded representations $\Lambda V=\sum \Lambda^{i} V$ and $\operatorname{Sym} V=\sum \operatorname{Sym}^{k} V$.)

Then

$$
\lambda_{t}(V) S_{t}(V)=1
$$

We will consider only representation rings of compact Lie groups and corresponding Lie algebras (reductive Lie algebras). Then it is sufficient to check an identity between natural operations for the group ring of $\mathrm{U}(1)$; this follows from the fact a character of a representation is determined by its restriction to the maximal torus. Using this remark we derive the above identity from the relation

$$
(1-x t)\left(1+x t+x^{2} t^{2}+\ldots\right)=1 .
$$

We define Adams operations $\Psi^{i}$ in terms of action on characters: operation $\Psi^{i}$ transforms a class function $\chi(g)$ into a class function $\chi\left(g^{i}\right)$. It is obvious that these operations are homomorphisms of representation ring:

$$
\begin{gathered}
\Psi^{i}(V+W)=\Psi^{i}(V)+\Psi^{i}(W), \\
\Psi^{i}(V W)=\Psi^{i}(V) \Psi^{i}(W) .
\end{gathered}
$$

It is clear also that

$$
\Psi^{k} \Psi^{l}(V)=\Psi^{k l}(V)
$$


The generating function $\Psi_{t}(V)=\sum \Psi^{i}(V) t^{i}$ can be expressed in the form

$$
\Psi_{t}(V)=-t \frac{d}{d t} \log \lambda_{t}(V)=t \frac{d}{d t} \log \sigma_{t}(V) .
$$

The proof of this relation also can be reduced to the consideration of the representation ring of $\mathrm{U}(1)$. Conversely, this relation allows us to express $\lambda^{k}$ and $\sigma^{k}$ in terms of Adams operations. In particular,

$$
\sigma_{t}(V)=\exp \left(-\sum \frac{\Psi^{k}(V) t^{k}}{k}\right)
$$

In terms of characters this formula can be written in the following way:

$$
\sum t^{k} \chi_{\operatorname{Sym}^{k} V}(g)=\exp \left(-\sum \frac{t^{k}}{k} \chi_{V}\left(g^{k}\right)\right)
$$

\section{References}

[1] E. Abdalla, M. Forger, and M. Jacques. Higher conservation laws for ten-dimensional supersymmetric Yang-Mills theory. Nuclear Phys. B, 308(1):198-220, 1988.

[2] M. Alexandrov, A. Schwarz, O. Zaboronsky, and M. Kontsevich. The geometry of the master equation and topological quantum field theory. Internat. J. Modern Phys. A, 12(2):1405-1429., 1997.

[3] E. A. Bergshoeff, A. Bilal, M. de Roo, and A. Sevrin. Supersymmetric nonabelian born-infeld revisited. JHEP, 0107(029), 2001.

[4] N. Berkovits. Covariant quantization of the superparticle using pure spinors. J. High Energy Phys., 09(016), 2001.

[5] N. Berkovits. Explaining the pure spinor formalism for the superstring. JHEP, 0801(065), 2008.

[6] N. Berkovits and P.S. Howe. The cohomology of superspace, pure spinors and invariant integrals. JHEP, 0806(046), 2008. 
[7] N. Berkovits and $\mathrm{N}$ Nekrasov. The character of pure spinors. Lett.Math.Phys., 74:75-109, 2005.

[8] R Bott. Homogeneous vector bundles. Annals of Mathematics, 66, No. 2, Sep., 1957(2):203-248, Sep 1957.

[9] D. Burghelea, Z. Fiedorowicz, and W. Gajda. Adams operations in hochschild and cyclic homology of de Rham algebra and free loop spaces. K-Theory, 4(3):269-287, 1991.

[10] H. Cartan and S. Eilenberg. Homological algebra. Princeton University Press, Princeton, NJ, 1999.

[11] M. Cederwall, B. E. W. Nilsson, and D. Tsimpis. D=10 super-yang-mills at o(alpha2). JHEP, 0107(042), 2001.

[12] M. Cederwall, B. E.W. Nilsson, and D. Tsimpis. The structure of maximally supersymmetric yang-mills theory: constraining higher-order corrections. JHEP, 0106(034), 2001.

[13] M. Cederwall, B.E.W. Nilsson, and D. Tsimpis. Spinorial cohomology and maximally supersymmetric theories. J. High Energy Phys., 0202(009), 2002.

[14] M. Cederwall, B.E.W. Nilsson, and D. Tsimpis. Spinorial cohomology of abelian $d=10$ super-yang-mills at $\alpha^{\prime 3}$. JHEP, 0211(023), 2002.

[15] C. Chevalley. The algebraic theory of spinors and Clifford algebras, volume 2 of collected works. Springer, 1997.

[16] A. Collinucci, M. de Roo, and M. G. C. Eenink. Supersymmetric Yang-Mills theory at order $\alpha^{\prime 3}$. JHEP 0206 (2002) 024, 0206(024), 2002.

[17] A. Connes. Noncommutative Geometry. Academic Press, San Diego, CA, 1994. 
[18] A. Connes and M. Dubois-Violette. Yang-mills and some related algebras. In Rigorous quantum field theory, volume 251 of Progr. Math., pages 65-78, Basel, 2007. Birkhäuser.

[19] A. Corti and M. Reid. Weighted grassmannians. In Algebraic geometry, pages 141-163. Walter de Gruyter and Co., Berlin, 2002.

[20] P. Deligne. Notes on spinors. In P. Etingof P. Deligne, D. Kazhdan, editor, Quantum fields and strings. A course for mathematicians, volume 1. AMS, 1999.

[21] D.Grayson and M. Stillman. Macaulay2. http://www.math.uiuc.edu/Macaulay2/.

[22] B. L. Feigin and B. L. Tsygan. Cyclic homology of algebras with quadratic relations, universal enveloping algebras and group algebras. In K-theory, arithmetic and geometry (Moscow, 1984-1986), volume 1289 of Lecture Notes in Math., pages 210-239. Springer, Berlin, 1987.

[23] P. Griffiths and J. Harris. Principles of algebraic geometry. Wiley,, 1978.

[24] M. Henneaux and C. Teitelboim. Quantization of gauge systems. Princeton University Press, Princeton, NJ, 1992.

[25] P. Howe. Pure spinors, function superspaces and supergravity theories in ten and eleven dimensions. Phys. Lett. B, 273:90-94, 1991.

[26] B. Keller. Derived invariance of higher structures on the Hochschild complex. preprint available at http://people.math.jussieu.fr/ keller/publ/dih.pdf.

[27] B. Keller. Invariance and localization for cyclic homology of dg algebras. J. Pure Appl. Algebra, 123(1-3):223-273, 1998.

[28] M. Kontsevich. Private communication. 
[29] T. Lada and J. Stasheff. Introduction to sh lie algebras for physicists. Int.J.Theor.Phys, 32:1087-1104, 1993.

[30] V. Lakshmibai and K.N. Raghavan. Standard monomial theory.. Invariant theoretic approach, volume 137 of Encyclopaedia of Mathematical Sciences. Springer, 2008.

[31] S. Mac Lane. Homology. Die Grundlehren der mathematischen Wissenschaften. Springer-Verlag, Berlin-Göttingen-Heidelberg, 1963.

[32] J.-L. Loday. Cyclic homology. Springer-Verlag, Berlin, 1998.

[33] M. Movshev. Cohomology of Yang-Mills algebras. J. Noncommut. Geom., $2(3): 353-404,2008$.

[34] M. Movshev and A. Schwarz. On maximally supersymmetric Yang-Mills theories. Nuclear Phys. B, 681(3):324-350, 2004.

[35] M. Movshev and A. Schwarz. Algebraic structure of Yang-Mills theory. In The unity of mathematics, volume 244 of Progr. Math., pages 473-523. Birkhäuser Boston, Boston, MA, 2006.

[36] M.V. Movshev. Deformation of maximally supersymmetric YangMills theory in dimensions 10. An algebraic approach, 2006. arXiv:hep-th/0601010v1.

[37] M.V. Movshev. Straightened law for quantum isotropic grassmannian $\operatorname{ogr}^{+}(5,10)$. arXiv:1110.5868v2 math-ph, 2011.

[38] M. Penkava and A.Schwarz. $A_{\infty}$ algebras and the cohomology of moduli spaces. In Lie groups and Lie algebras: E. B. Dynkin's Seminar, volume 169 of Amer. Math. Soc. Transl. Ser. 2, pages 91-107. Amer. Math. Soc., Providence, RI,, 1995.

[39] A. Polishchuk and L. Positselski. Quadratic algebras, volume 37 of Univ. Lecture Ser. Amer. Math. Soc., Providence, RI, 2005. 
[40] S. Priddy. Koszul resolutions. Trans. Amer. Math. Soc., 152:39—60, 1970.

[41] A. Schwarz. Semiclassical approximation in Batalin-Vilkovisky formalism. Commun. Math. Phys, 158:373-396, 1993.

[42] J Stasheff. The (secret?) homological algebra of the Batalin-Vilkovisky approach. In Secondary calculus and cohomological physics (Moscow, 1997), volume 219 of Contemp. Math., pages 195-210, Providence, RI, 1998. Amer. Math. Soc.

[43] E. B Vinberg and A. L. Onishchik. Seminar on Lie groups and algebraic groups. Springer series in Soviet mathematics. Springer Verlag, Berlin, New York, 1990.

[44] C. Weibel. An Introduction to Homological Algebra. Cambridge Studies in Advanced Mathematics. Cambridge University Press, October 271995.

[45] B. Zumino. Cohomology of gauge groups: cocycles and Schwinger terms. Nucl. Phys. B, 253:477, 1985. 